\title{
Relevance of accurate Monte Carlo modeling in nuclear medical imaging
}

\author{
Habib Zaidia) \\ Division of Nuclear Medicine, Geneva University Hospital, CH-1211 Geneva, Switzerland
}

(Received 23 June 1998; accepted for publication 3 February 1999)

\begin{abstract}
Monte Carlo techniques have become popular in different areas of medical physics with advantage of powerful computing systems. In particular, they have been extensively applied to simulate processes involving random behavior and to quantify physical parameters that are difficult or even impossible to calculate by experimental measurements. Recent nuclear medical imaging innovations such as single-photon emission computed tomography (SPECT), positron emission tomography (PET), and multiple emission tomography (MET) are ideal for Monte Carlo modeling techniques because of the stochastic nature of radiation emission, transport and detection processes. Factors which have contributed to the wider use include improved models of radiation transport processes, the practicality of application with the development of acceleration schemes and the improved speed of computers. In this paper we present a derivation and methodological basis for this approach and critically review their areas of application in nuclear imaging. An overview of existing simulation programs is provided and illustrated with examples of some useful features of such sophisticated tools in connection with common computing facilities and more powerful multiple-processor parallel processing systems. Current and future trends in the field are also discussed. (C) 1999 American Association of Physicists in Medicine. [S0094-2405(99)01904-5]
\end{abstract}

Key words: Monte Carlo simulations, SPECT, PET, scatter, detectors

\section{INTRODUCTION AND OVERVIEW}

Recent developments in nuclear medicine instrumentation and multiple-processor parallel processing systems have created a need for a review of the opportunities for Monte Carlo simulation in nuclear medicine imaging. One of the aims of the medical physicist involved in nuclear medical imaging research is to optimize the design of imaging systems and to improve the quality and quantitative accuracy of reconstructed images. Several factors affect the image quality and the accuracy of the data obtained from a nuclear medicine scan. These include the physical properties of the detectors, collimator and gantry design, attenuation and scatter compensation and reconstruction algorithms. ${ }^{1,2}$ Integrating improvements in these with current tracers and sensitive and specific tracers under development will provide major advantages to the general nuclear medicine clinician and research investigator (Fig. 1). Mathematical modeling is necessary for the assessment of various parameters in nuclear medical imaging systems since no analytical solution is possible when solving the transport equation describing the interaction of photons with nonuniformly attenuating body structures and complex detector geometries.

The Monte Carlo method is widely used for solving problems involving statistical processes and is very useful in medical physics due to the stochastic nature of radiation emission, transport and detection processes. The method is very useful for complex problems that cannot be modeled by computer codes using deterministic methods or when experimental measurements may be impractical. The Monte Carlo method was named by Von Neumann ${ }^{3}$ because of the similarity of statistical simulation to games of chance, and because the city in the Monaco principality was a center for gambling and similar pursuits. Von Neumann, Ulam and Fermi applied the method towards neutron diffusion problems in the Manhattan Project at Los Alamos during World War II. But even at an early stage of these investigations, von Neumann and Ulam refined this particular "Russian roulette" and "splitting" methods. However, the systematic development of these ideas had to await the work of Kahn and Harris in 1948. ${ }^{4}$ During the same year, Fermi, Metropolis and Ulam obtained Monte Carlo estimates for the eigenvalues of the Schrodinger equation. Uses of Monte Carlo methods have been many and varied since that time. The applications of the Monte Carlo method in medical physics were few before the review paper by Raeside. ${ }^{5}$ Since that time, there has been an increasing number of applications of Monte Carlo techniques to problems in this field thanks to the several books ${ }^{6-9}$ and comprehensive review papers ${ }^{5,10-12}$ describing the principles of the Monte Carlo method and its applications in medical physics.

There has been an enormous increase and interest in the use of Monte Carlo techniques in all aspects of nuclear imaging, including planar imaging, ${ }^{13}$ single-photon emission computed tomography (SPECT) ${ }^{14-18}$ positron emission tomography $(\mathrm{PET})^{19-22}$ and multiple emission tomography (MET) ${ }^{23}$ However, due to computer limitations, the method has not yet fully lived up to its potential. With the advent of high-speed supercomputers, the field has received increased attention, particularly with parallel algorithms which have much higher execution rates. Our main purpose in this paper is to present a framework for applying Monte Carlo simulations for a wide range of problems in nuclear medical imaging. Emphasis is given to applications where photon and/or electron transport in matter is simulated. Some computa- 


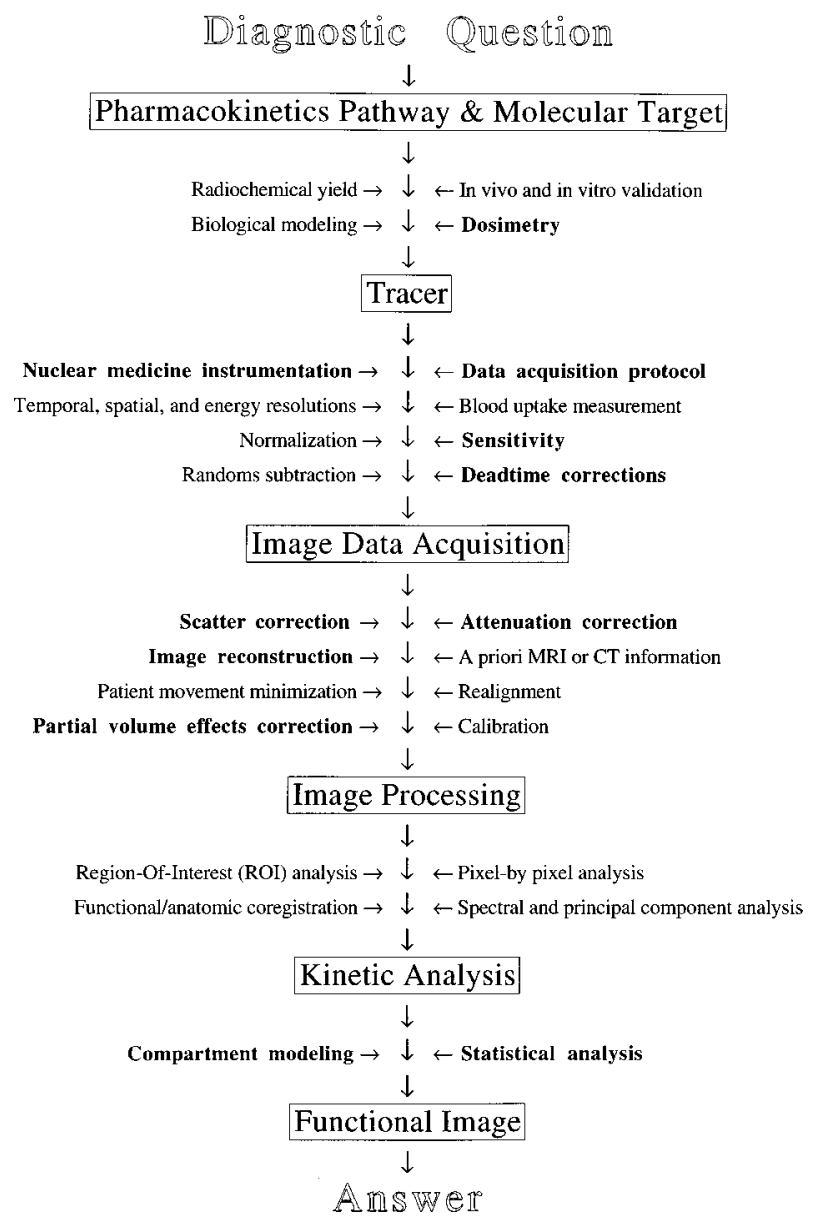

FIG. 1. Scientific and technical strategy for recording accurate functional images. In bold, the parts where Monte Carlo simulation plays an important role (adapted from an illustration by Professor Terry Jones, MRC).

tional aspects of the Monte Carlo method, mainly related to random numbers, sampling and variance reduction are discussed. Basic aspects of nuclear medicine instrumentation are reviewed, followed by the presentation of potential applications of Monte Carlo techniques in different areas of nuclear imaging such as detector modeling and systems design, image reconstruction and scatter correction techniques, internal dosimetry and pharmacokinetic modeling. Widely used Monte Carlo codes in connection with computing facilities, vectorized and parallel implementations are described. Current trends and some strategies for future development in the field are also discussed.

\section{THE MONTE CARLO METHOD: THEORY AND COMPUTATIONAL ISSUES}

Numerical methods that are known as Monte Carlo methods can be loosely described as statistical simulation methods, where statistical simulation is defined in quite general terms to be any method that utilizes sequences of random numbers to perform the simulation. A detailed description of the general principles of the Monte Carlo method is given in a number of publications, ${ }^{5,11,24,25}$ and will not be repeated here. Figure 2 illustrates the idea of Monte Carlo or statisti-
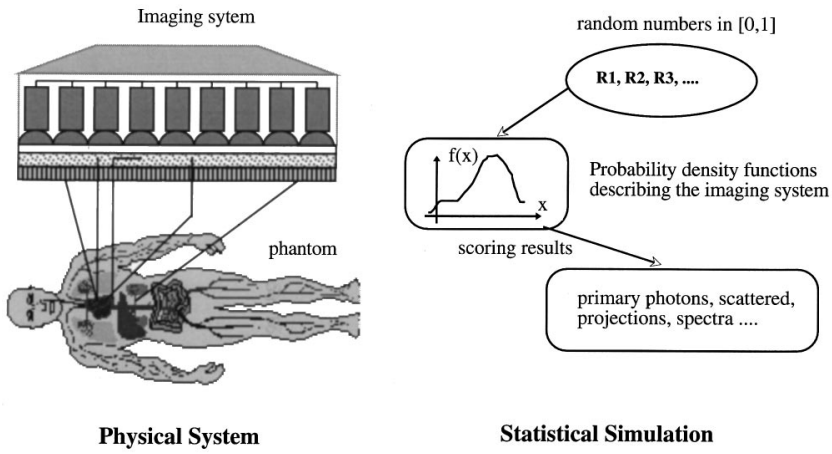

FIG. 2. Principles of Monte Carlo simulation of an imaging system.

cal simulation as applied to an imaging system. Assuming that the behavior of the imaging system can be described by probability density functions (pdf's), then the Monte Carlo simulation can proceed by sampling from these pdf's, which necessitates a fast and effective way to generate random numbers uniformly distributed on the interval $[0,1]$. Photon emissions are generated within the phantom and are transported by sampling from pdf's through the scattering medium and detection system until they are absorbed or escape the volume of interest without hitting the crystal. The outcomes of these random samplings, or trials, must be accumulated or tallied in an appropriate manner to produce the desired result, but the essential characteristic of Monte Carlo is the use of random sampling techniques to arrive at a solution of the physical problem.

The major components of a Monte Carlo method are briefly described below. These components comprise the foundation of most Monte Carlo applications. The following sections will explore them in more detail. An understanding of these major components will provide a sound foundation for the developer to construct his own Monte Carlo method, although the physics and mathematics of nuclear imaging are well beyond the scope of this paper. The primary components of a Monte Carlo simulation method include the following.

(i) Probability density functions (pdf's): the physical system must be described by a set of pdf's.

(ii) Random number generator: a source of random numbers uniformly distributed on the unit interval must be available.

(iii) Sampling rule: a prescription for sampling from the specified pdf's.

(iv) Scoring: the outcomes must be accumulated into overall tallies or scores for the quantities of interest.

(v) Error estimation: an estimate of the statistical error (variance) as a function of the number of trials and other quantities must be determined.

(vi) Variance reduction techniques: methods for reducing the variance in the estimated solution to reduce the computational time for Monte Carlo simulation.

(vii) Parallelization and vectorization algorithms to allow 
Monte Carlo methods to be implemented efficiently on advanced computer architectures.

\section{A. Random numbers generation}

Computational studies requiring the generation of random numbers are becoming increasingly common. All random number generators (RNG) are based upon specific mathematical algorithms, which are repeatable. As such, the numbers are just pseudo-random. Here, for simplicity, we shall term them just "random" numbers. Formally, random is defined as exhibiting "true" randomness, such as the time between "tics" from a Geiger counter exposed to a radioactive element. Pseudo-random is defined as having the appearance of randomness, but nevertheless exhibiting a specific, repeatable pattern. Quasi-random is defined as filling the solution space sequentially (in fact, these sequences are not at all random, they are just comprehensive at a preset level of granularity). Monte Carlo methods make extensive use of random numbers to control the decision making when a physical event has a number of possible results. The RNG is always one of the most crucial subroutines in any Monte Carlo-based simulation code. ${ }^{24} \mathrm{~A}$ large number of generators are readily available, ${ }^{26}$ and many of these are suitable for the implementation on any computer system, ${ }^{27}$ since today there is no significant distinction in floating point processing capabilities between a modern desktop and a mainframe computer. A typical simulation uses from $10^{7}$ to $10^{12}$ random numbers, and subtle correlations between these numbers could lead to significant errors. ${ }^{28}$ The largest uncertainties are typically due more to approximations arising in the formulation of the model than those caused by the lack of randomness in the RNG. Mathematically speaking, the sequence of random numbers used to effect a Monte Carlo model should possess the following properties. ${ }^{29}$

(i) Uncorrelated sequences: the sequences of random numbers should be serially uncorrelated. Most especially, $n$-tuples of random numbers should be independent of one another.

(ii) Long period: ideally, the generator should not repeat; practically, the repetition should occur only after the generation of a very large set of random numbers.

(iii) Uniformity: the sequence of random numbers should be uniform, and unbiased. That is, suppose we define $n$-tuples $\mu_{i}^{n}=\left(u_{i+1}, \ldots, u_{i+n}\right)$ and divide the $n$-dimensional unit hypercube into many equal subvolumes. A sequence is uniform if in the limit of an infinite sequence all the sub-volumes have an equal number of occurrences of random $n$-tuples.

(iv) Reproducibility: when debugging programs, it is necessary to repeat the calculations to find out how the errors occurred. The feature of reproducibility is also helpful while porting the program to a different machine.

(v) Speed: It is of course desirable to generate the random numbers fast.

(vi) Parallelization: The generator used on vector machines should be vectorizable, with low overhead. On massively parallel architectures, the processors should not have to communicate among themselves, except perhaps during initialization.

Although powerful RNGs have been suggested including shift register, inversive congruentional, combinatorial and "intelligent" methods such as those implemented in the MCNP code ${ }^{30}$ the most commonly used generator is the linear congruential RNG (LCRNG). ${ }^{31}$ Recently, Monte Carlo researchers have become aware of the advantages of lagged Fibonacci series (LFRNG). With extremely long periods, they are generally faster than LCRNG and have excellent statistical properties. ${ }^{32}$ Those generators are briefly described below.

\section{Linear congruential generators}

The LCRNG has the form ${ }^{31}$

$$
u_{n+1}=a\left(u_{n}+c\right) \bmod (m) \text {, }
$$

where $m$ is the modulus, $a$ is the multiplier and $c$ is the additive constant or addend. The size of the modulus constrains the period, and is usually chosen to be either prime or a power of $2 .{ }^{33}$ An important subset of LCRNG is obtained by setting $c=0$ in Eq. (1), which defines the multiplicative linear congruential RNG(MLCRNG). This generator (with $m$ a power of 2 and $c=0$ ) is the de facto standard included with FORTRAN and C compilers. ${ }^{34}$ One of the biggest disadvantages to using a power of 2 modulus is that the least significant bits of the integers produced by these LCRNGs have extremely short periods. For example, $\mu_{n} \bmod \left(2^{j}\right)$ will have a period of $2^{j}{ }^{33}$ In particular, this means the least-significant bit of the LCRNG will alternate between 0 and 1 . Some cautions to the programmer are in order: (i) the bits of $\mu_{n}$ should not be partitioned to make several random numbers since the higher order bits are much more random than the lower order bits; (ii) the power of 2 modulus in batches of powers of 2 should be avoided; (iii) RNGs with large modulus are preferable to ones with small modulus. Not only is the period longer, but the correlations are lower. In particular, one should not use a 32 bit modulus for applications requiring a high resolution in the random numbers. In spite of this known defect of power of 2 LCRNGs, 48 bit multipliers (and higher) have passed many very stringent randomness tests.

The initial seed should be set to a constant initial value, such as a large prime number (it should be odd, as this will satisfy period conditions for any modulus). Otherwise, the initial seed should be set to a "random" odd value. Anderson $^{35}$ recommends setting the initial seed to the following integer:

$$
\begin{aligned}
u_{0}= & i \mathrm{yr}+100 \times(i \text { month }-1+12 \times(i \text { day }-1+31 \\
& \times(i \text { hour }+24 \times(i \text { min }+60 \times i \text { sec })))),
\end{aligned}
$$

where the variables on the right-hand side are the integer values of the date and time. Note that the year is 2 digits long, i.e., the domain of $i \mathrm{yr}$ is [0-99]. However, it may be preferable to introduce the maximum variation in the seed 
into the least significant bits by using the second of this century, rather than the most significant bits. The following equation is preferable:

$$
\begin{aligned}
u_{0}= & i \sec +60 \times(i \min +60 \times(i \text { hour }+24 \\
& \times(i \text { day }-1+31 \times(i \text { month }-1+12 i \mathrm{yr}))))
\end{aligned}
$$

Generally, LCRNGs are best parallelized by parameterizing the iteration process, either through the multiplier or the additive constant. Based on the modulus, different parametrizations have been tried. ${ }^{35}$

\section{Lagged-Fibonacci generators}

The lagged-Fibonacci series RNG (LFRNG) have the following general form: ${ }^{26}$

$$
u_{n}=u_{n-l} \otimes u_{n-k} \bmod (m), \quad l>k,
$$

where $\otimes$ may be one of the following binary arithmetic operators $+,-, *, l$ and $k$ are the lags and $m$ is a power of $2\left(m=2^{P}\right)$. In recent years the additive lagged-Fibonacci RNG (ALFRNG) has become a popular generator for serial as well as scaleable parallel machines ${ }^{36}$ because it is easy to implement, it is cheap to compute and it does well on standard statistical tests, especially when the lag $k$ is sufficiently high (such as $k=1279$ ). The maximal period of the ALFRNG is $\left(2^{k}-1\right) 2^{p-1}$ and has $2^{(k-1)(p-1)}$ different fullperiod cycles. ${ }^{37}$ Another advantage of the ALFRNG is that one can implement these generators directly in a floatingpoint to avoid the conversion from an integer to a floatingpoint that accompanies the use of other generators. However, some care should be taken in the implementation to avoid floating-point round-off errors.

Instead, the ALFRNG can be parameterized through its initial values because of the tremendous number of different cycles. Different streams are produced by assigning each stream a different cycle. An elegant seeding algorithm that accomplishes this is described by Mascagni. ${ }^{36}$ An interesting cousin of the ALFRNG is the multiplicative laggedFibonacci RNG (MLFRNG). While this generator has a maximal-period $\left(2^{k}-1\right) 2^{p-3}$, which is a quarter the length of the corresponding ALFRNG, it has empirical properties considered to be superior to ALFRNGs. ${ }^{26}$ Of interest for parallel computing is that a parameterization analogous to that of the ALFRNG exists for the MLFRNG. This latter algorithm was used for generating uniformly distributed random numbers on a parallel computer based on the MIMD principle. ${ }^{38}$ The sequence of 24 bit random numbers has a period of about $2^{144}$ and has passed stringent statistical tests for randomness and independence. ${ }^{32}$

\section{B. Photon transport}

For radiation transport problems, the computational model includes geometry and material specifications. ${ }^{39} \mathrm{Ev}-$ ery computer code contains a database of experimentally obtained quantities, known as cross-sections, that determine the probability of a particle interacting with the medium through which it is transported. Every cross-section is peculiar to the type and energy of the incident particle and to the kind of interaction it undergoes. These partial cross-sections are summed to form the total cross-section; the ratio of the partial cross-section to the total cross-section gives the probability of this particular interaction occurring. Cross-section data for the interaction types of interest must be supplied for each material present. The model also consists of algorithms used to compute the result of interactions (changes in particle energy, direction, etc.) based on the physical principles that describe the interaction of radiation with matter and the cross-section data provided. Therefore, it is extremely important to use an accurate transport model as the Monte Carlo result is only as valid as the data supplied.

When a photon (having an energy below $1 \mathrm{MeV}$ ) passes through matter, any of the three interaction processes (photoelectric, incoherent scattering, coherent scattering) may occur. The probability of a photon of a given energy $E$ undergoing absorption or scattering when traversing a layer of material $Z$ can be expressed quantitatively in terms of a linear attenuation coefficient $\mu\left(\mathrm{cm}^{-1}\right)$ which is dependent on the material's density, $\rho\left(\mathrm{g} . \mathrm{cm}^{-3}\right)$,

$$
\mu=\mu_{\text {photo }}+\mu_{\text {incoh }}+\mu_{\text {coh }} .
$$

In the case of photoelectric absorption, the total photon energy is transferred to an atomic electron and the random walk is terminated. In an incoherent photon interaction, a fraction of the photon energy is transferred to the atomic electron. The direction of the scattered photon is changed to conserve the total momentum of the interaction. The Klein-Nishina expression for the differential cross-section per electron for an incoherent interaction is used to sample the energy and the polar angle of the incoherently scattered photon. ${ }^{40}$ The coherent scattering only results in a change in the direction of the photon since the momentum change is transferred to the whole atom. The kinetic energy loss of the photon is negligible. Coherent scattering of a photon could be generated using the random number composition and rejection technique ${ }^{4}$ to sample the momentum of the scattered photon and the scattering angle according to the form factor distribution.

It is common to neglect coherent scattering in PET Monte Carlo simulation of photon transport because of its low contribution to the total cross-section at $511 \mathrm{keV}$. In the following examples, the relative importance of the various processes involved in the energy range of interest (below 1 $\mathrm{MeV}$ ) are considered for some compounds and mixtures used in nuclear medicine to justify some of the approximations made in Monte Carlo codes. Figure 3 illustrates the relative strengths of the photon interactions versus energy for water, cortical bone, sodium iodide (NaI) and bismuth germanate (BGO), respectively. For water, a moderately low- $Z$ material, we note two distinct regions of single interaction dominance: photoelectric below and incoherent above 20 $\mathrm{keV}$. The almost order of magnitude depression of the coherent contribution is some justification for the approximations discussed. The coherent contribution to the total crosssection is less than $1 \%$ for energies above $250 \mathrm{keV}$. However, this contribution is in the order of $7 \%$ for high- $Z$ ma- 
$\mathrm{H} 2 \mathrm{O}$

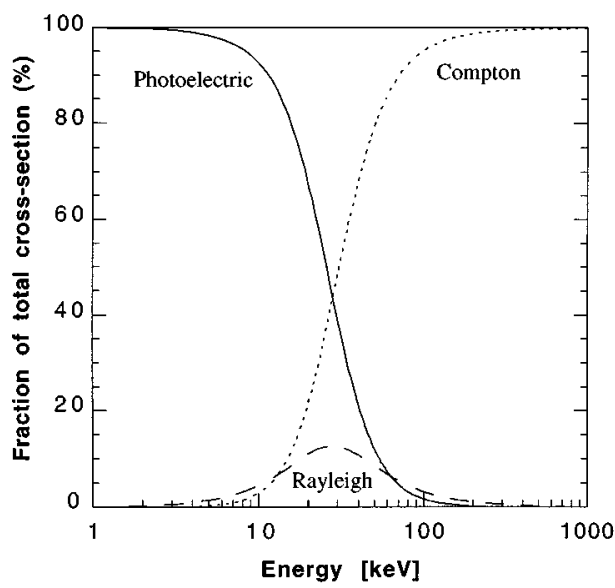

Nal

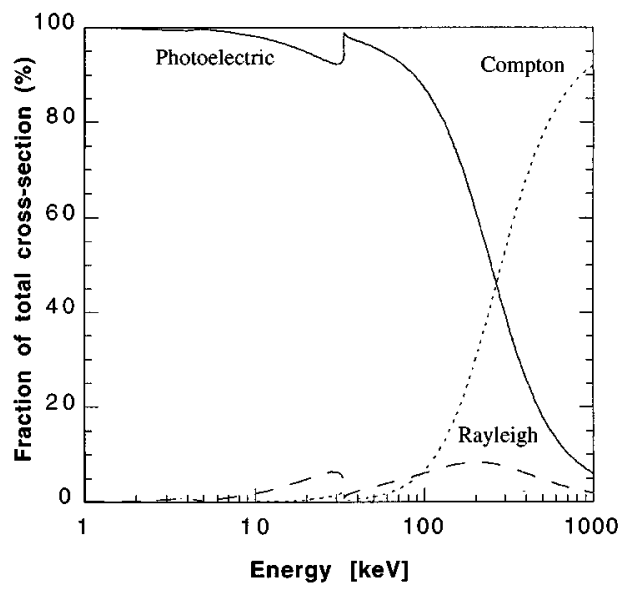

Cortical bone

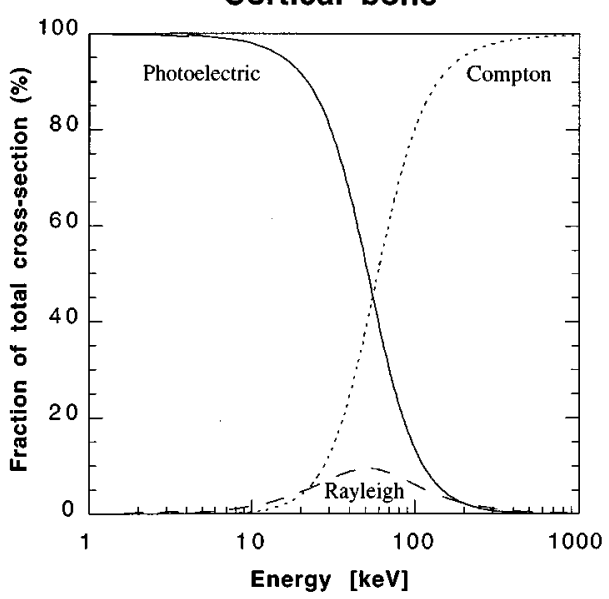

BGO

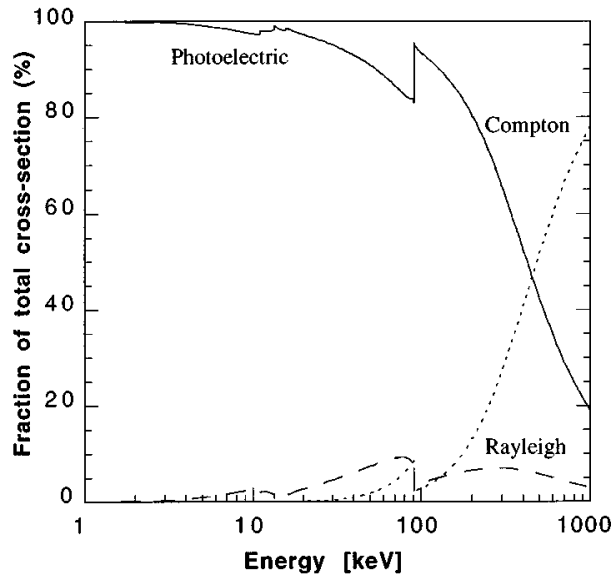

FIG. 3. Components of photon crosssections for different tissues $\left(\mathrm{H}_{2} \mathrm{O}\right.$ and cortical bone) and detector materials ( $\mathrm{NaI}$ and $\mathrm{BGO}$ ) of interest in nuclear imaging, illustrating the relative contribution of each process. terials like BGO. Therefore, efforts should be made to treat the coherent scattering process adequately for detector materials. In a recent investigation, photon cross-section libraries (NIST, PHOTX) $)^{41,42}$ and parametrizations implemented in simulation packages (GEANT, PETSIM) ${ }^{43,44}$ were compared to the recent library provided by the Lawrence Livermore National Laboratory (EPDL97) ${ }^{45}$ for energies from $1 \mathrm{keV}$ to $1 \mathrm{MeV}$ for a few human tissues and detector materials of interest in nuclear imaging. ${ }^{46}$ The cross-section data for mixtures and compounds are obtained from the equation:

$$
\mu=\rho \sum_{i} w_{i}(\mu / \rho)_{i},
$$

where $\rho$ is the density of the material, $w_{i}$ the fraction by weight of the $i$ th atomic constituent, as specified in ICRU Report $44^{47}$ and $(\mu / \rho)_{i}$ the mass attenuation coefficients. Different photon cross-section libraries show quite large variations as compared to the most recent EPDL97 data files. It is recommended that Monte Carlo developers only use the most recent version of this library. ${ }^{46}$

A calculation of the distances between interactions in a medium are performed by sampling from the exponential attenuation distribution [Eq. (10) below]. Different techniques have been proposed to improve the computation speed when sampling from the probability distributions. They are described in more detail in Secs. II D and IIE.

\section{Electron transport}

In principle, electron transport should be included when simulating the complete electromagnetic cascade (microscopic techniques). However, the large number of interactions that may occur during electron slowing down makes it unrealistic to simulate all the physical interactions (macroscopic techniques). ${ }^{10}$ Secondary electrons are generally assumed to deposit all their energy at the point of interaction because of the low energies involved in nuclear medicine, and therefore the short ranges of the electrons generated and their negligible bremsstrahlung production. Therefore, electron transport has not received particular attention in nuclear imaging applications of the Monte Carlo method. However, a number of investigators considered this effect mainly for dosimetry calculations. ${ }^{48-50}$

Most existing electron transport algorithms are based on the multiple collision models for scattering and energy loss. The complexity of the techniques used in microscopic models varies considerably, although a common approach is to neglect bremsstrahlung interactions. Simple models are 
based on the simulation of all the scattering events, calculating the step length between consecutive collisions with the elastic mean-free path. Energy losses are determined from the Bethe theory of stopping power and an approximation included to account for the energy-loss straggling. This model has been improved later by taking inelastic collisions into account. ${ }^{10}$ Macroscopic techniques classify the physical interactions of electrons into groups that provide an overall picture of the physical process. Berger ${ }^{51}$ divided electron transport algorithms into two broad classes (class I and class II) distinguished by how they treat individual interactions that lead to the energy losses of the primary electrons and the production of bremsstrahlung photons and/or knock-on electrons. The condensed-history technique for electron transport has been reviewed ${ }^{51}$ and comparisons of class I with class II algorithms and of Goudsmit and Saunderson multiplescattering theory have also been made. ${ }^{10,40}$ The Moliere theory contains a small-angle approximation ${ }^{52}$ and requires a certain minimum number of scattering events to occur, whereas the Goudsmit and Saunderson theory is exact for a single-scattering cross-section. It has been shown, however, that the effects of the small-angle approximation can be compensated. ${ }^{51}$ An improved model for multiple scattering into the voxel Monte Carlo algorithm comparable in accuracy with the Parameter Reduced Electron-Step Transport Algorithm (PRESTA) ${ }^{53}$ has been developed recently. ${ }^{54}$

A systematic error is introduced in low energy transport when the algorithm does not account for the change in a discrete interaction cross-section with energy. ${ }^{55}$ To overcome this problem, $\mathrm{Ma}^{56}$ developed an algorithm to account properly for the change in an electron discrete interaction cross-section as a function of energy for low energy electron transport.

\section{Analog sampling}

Analog Monte Carlo attempts to simulate the full statistic development of the electromagnetic cascade. If we assume that a large number of particle histories, $N$, are included in a batch, the individual batch estimates can be considered as drawn from a normal distribution. For a given calculation, the estimated uncertainty is proportional to the inverse of the square root of the number of histories simulated. The efficiency $\epsilon$ of a Monte Carlo calculation can therefore be defined as ${ }^{57}$

$$
\epsilon=\frac{1}{\sigma^{2} T},
$$

where $T$ is the calculation time to obtain a variance estimate $\sigma^{2}$. For large $N, \epsilon$ should be constant as long as the calculation technique remains the same.

As described earlier, the imaging system can be described in terms of pdf's. These pdf's, supplemented by additional computations, describe the evolution of the overall system, whether in space, energy, time or even some higher dimensional phase space. The goal of the Monte Carlo method is to simulate the imaging system by random sampling from these pdf's and by performing the necessary supplementary com- putations needed to describe the system evolution. In essence, the physics and mathematics are replaced by random sampling of possible states from pdf's that describe the system. Thus, it is frequently necessary to sample some physical event, the probability of which is described by a known pdf. Examples include the distance to the next interaction and the energy of a scattered photon. Let $x$ be the physical quantity to be selected and $f(x)$ the pdf. Among the properties of the pdf is that it is integrable and non-negative. Assume that the domain of $f(x)$ is the interval $\left[x_{\min }, x_{\max }\right]$ and that it is normalized to unit area. The cumulative distribution function $F(x)$ of the frequency function $f(x)$ gives the probability that the random variable $\tau$ is less or equal to $x$. It is defined as

$$
F(x) \equiv \operatorname{probability}(\tau \leqslant x)=\int_{x_{\min }}^{x} f(\tau) d \tau .
$$

A stochastic variable can be sampled by the use of uniformly distributed random numbers $\mathbf{R}$ in the range [0-1] using one of the techniques described below.

\section{Direct method}

This method can be used if the inverse of the cumulative distribution function $F^{-1}(x)$ is easily obtainable. Since $F(x)$ is uniformly distributed in [0-1], the sampled value of $x$ could be obtained by substituting $F(x)$ in Eq. (8) by a uniform random number $\mathbf{R}$, that is, $x=F^{-1}(\mathbf{R})$. A practical example of using this technique is the calculation of the distance to the next interaction vertex. The inversion is not always possible, but in many important cases the inverse is readily obtained.

\section{Rejection method}

Another method of performing this when it is too complicated to obtain the inverse of the distribution function is to use the rejection technique, ${ }^{4}$ which follows the following steps: (i) define a normalized function $f^{\prime}(x)=f(x) / f_{\max }(x)$, where $f_{\max }(x)$ is the maximum value of $f(x)$; (ii) sample two uniformly distributed random numbers $\mathbf{R}_{\mathbf{1}}$ and $\mathbf{R}_{\mathbf{2}}$; (iii) calculate $x$ using the equation $x=x_{\min }+\mathbf{R}_{1}\left(x_{\max }-x_{\min }\right)$; and (iv) if $\mathbf{R}_{\mathbf{2}}$ is less than or equal to $f^{\prime}(x)$, then $x$ is accepted as a sampled value; otherwise a new value of $x$ is sampled.

Over a large number of samples, this technique will yield a set of values of $x$ within the required distribution. It does, however, require two random numbers per trial and many trials may be required depending on the area under of the curve of $f(x)$. A typical example of using this technique is the photon energy and scattering angle resulting from incoherent scattering.

\section{Mixed methods}

When the previous two methods are impractical, the mixed method that combines the two may be used. ${ }^{57}$ Assume that the pdf can be factored as follows:

$$
f(x)=h(x) \cdot g(x),
$$


where $h(x)$ is an invertible function and $g(x)$ is relatively flat but contains most of the mathematical complexity. The method consists of the following steps: (i) normalize $h(x)$ producing $h^{\prime}(x)$ such that $\int_{x_{\min }}^{x_{\max }} h^{\prime}(x) d x=1$; (ii) normalize $g(x)$ producing $g^{\prime}(x)$ such that $g^{\prime}(x) \leqslant 1$ for $x$ in $\left[x_{\min }, x_{\max }\right]$; (iii) use the direct method to select an $x$ using $h^{\prime}(x)$ as the pdf; (iv) use $x$ and apply the rejection method using $g^{\prime}(x)$, i.e., choose a random number $\mathbf{R}$, if $g^{\prime}(x) \leqslant \mathbf{R}$, accept $x$; otherwise go back to step (iii).

\section{E. Nonanalog sampling "variance reduction techniques"}

A direct Monte Carlo simulation using true probability functions may require an unacceptable long time to produce statistically relevant results. Photons emission is isotropic, so directional parameters may be sampled uniformly within their individual ranges. Nuclear imaging systems have a low geometrical efficiency because of the small solid angle defined by the collimator and/or the small axial aperture. Therefore, the calculation would be very ineffective in terms of required computing time. ${ }^{58}$ It is thus desirable to bias the sampling (nonanalog sampling) by introducing different types of importance sampling and other variance reduction techniques to improve the computational efficiency of the Monte Carlo method. ${ }^{59}$ The results obtained by nonanalog simulation are, however, biased by the variance reduction technique and a correction for this is required. A particle history weight, $W$, is introduced, which describes the probability of the particle following the current path. This weight is calculated for each particle history, and used in the calculation of the results. If an event occurs, the weight $W$ is added to the counter rather than incrementing the counter by one unit. Bielajew and Rogers ${ }^{57}$ divided variance reduction techniques in three categories: those that concern photon transport only, those that concern electron transport only, and other more general methods. The most useful techniques are described below.

\section{Photon-specific methods}

Interaction forcing. In an analog Monte Carlo simulation, photons are tracked through the object until they either escape the object, are absorbed or their energy drops below a selected threshold. The probability function for a photon interaction is given by

$$
p(x)=\mu e^{-\mu x} .
$$

The probability that a photon will travel a distance $d$ or less is given by

$$
p(d)=\int_{0}^{d} \mu e^{-\mu x} d x=1-e^{-\mu d} .
$$

To sample the pathlength, a uniform random number $\mathbf{R}$ is substituted for $p(d)$ and the problem is solved for $d$ :

$$
d=-\frac{\log (1-\mathbf{R})}{\mu} .
$$

Since the maximum distance $d_{\max }$, the photon travels before interaction is infinite, and the number of photon mean free paths across the geometry in any practical situation is finite, there is a large probability that photons leave the geometry of interest without interacting. To increase the statistical accuracy in the imparted energy calculation, we force the photons to interact by assigning $d_{\max }$ a finite distance, e.g., the thickness of the detector being simulated. ${ }^{57} \mathrm{~A}$ true distributed photon pathlength $d$ within $d_{\max }$ can be sampled from the equation

$$
d=-\frac{1}{\mu} \ln \left(1-\mathbf{R}\left[1-e^{-\mu d_{\max }}\right]\right) .
$$

The photon's weight must be multiplied by the interaction probability,

$$
W_{n+1}=W_{n}\left[1-e^{-\mu d_{\max }}\right] .
$$

In emission computed tomography, the photon is allowed to interact through coherent or incoherent interactions only within the phantom since photoabsorption does not contribute to energy imparted in the crystal. The weight is then multiplied by the probability for the photon being scattered:

$$
W_{n+1}=W_{n}\left[\frac{\mu_{\text {incoh }}+\mu_{\text {coh }}}{\mu}\right],
$$

where $\mu_{\text {incoh }}$ and $\mu_{\text {coh }}$ are the cross-section data for incoherent and coherent scattering, respectively, and $\mu$ is the total linear attenuation coefficient.

Stratification. Stratification refers to the process of determining the frequencies with which the various regions of state space are used to start a particle. ${ }^{60}$ The solid angle of acceptance of the detector array, $\Omega_{\max }$, is small due to collimation and to the size of the detector array itself. This results in significant computational inefficiencies with analog Monte Carlo simulation, because only a few percent of the photons generated and tracked will actually be detected. The goal of stratification is to simulate only photons that are emitted in directions within the solid angle, which can be calculated from the maximum acceptance angle, $\theta_{\max }$, which, in turn, can be estimated from the dimensions of the phantom and the detection system. The solid angle does not change in magnitude when simulating source locations offcenter. The photon escaping from the phantom is either primary or scattered. If the photon happens to be a primary photon, its direction within the solid angle could be sampled from

$$
\cos (\theta)=1-\mathbf{R}\left[1-\cos \theta_{\max }\right] .
$$

In this case, the weight is multiplied by the probability of escape without interaction in the solid angle $\Omega_{\max }$,

$$
W_{n+1}=W_{n} \frac{\left[1-\cos \theta_{\max }\right]}{2} .
$$

Exponential transform, russian roulette and particle splitting. The exponential transform is a variance reduction technique used to bias the sampling procedure to give more interactions in the regions of interest and thus improve the 
efficiency of the calculation for those regions. To implement this method, the distance to the next interaction in number of mean free paths, $d_{\lambda}$, should be sampled from ${ }^{57}$

$$
d_{\lambda}=-\frac{\ln (\mathbf{R})}{(1-C \cos \theta)},
$$

where $C$ is a parameter that adjusts the magnitude of the scaling and $\theta$ the angle of the photon with respect to the direction of interest. The new weighting factor is given by

$$
W_{n+1}=W_{n} \frac{\exp \left(-d_{\lambda} C \cos \theta\right)}{(1-C \cos \theta)} .
$$

Note that the new weighting factor is dependent on $d_{\lambda}$. If $0<C<1$, the particle pathlength is stretched in the forward direction, which is used for shielding problems. For $-1<C<0$, the average distance to the next interaction is shortened in the forward direction, which is used for surface problems. For $C=0$, we recover the unbiased sampling. The optimal choice of this parameter is dependent on the problem to be solved. The general guideline is to avoid to use large weighting factors because they may increase the variance. ${ }^{57}$

Russian roulette and splitting are often used together with the exponential transform although they are still effective when used independently. In Russian roulette, a random number is selected and compared to a threshold, $\lambda$. If the random number turns out to be smaller than $\lambda$, the particle is allowed to survive but the weight should be updated accordingly, $W_{n+1}=W_{n} / \lambda$. In particle splitting, a particle coming from a region of interest can be divided into $N$ particles, each having a new weighting, $W_{n+1}=W_{n} / N$.

\section{Electron-specific methods}

Electron range rejection. A fundamental difference between the transport of photons and electrons in a condensedhistory simulation code is that photons travel relatively long distances before interacting while electron tracks are interrupted not only by geometrical boundaries but also by multiple scattering "steps." A large amount of simulation time is spent on checking boundaries and selecting deflection angles and so on. Electron range rejection means that any electrons with their residual range smaller than the distance to the nearest boundary or to the region of interest in the simulation will be terminated to save computing time. Different methods have been suggested for electron range rejection. The reduced interrogation of geometry (RIG) method calculates the distance to the nearest boundary and compare it to the maximum multiple-scattering step length. If the electron cannot reach any of the boundaries during this step, the boundary checking routine will not be called and this will save computing time. Another method called "disregard within a zone"' is usually used with RIG to further speed up the simulation. It consists of disregarding electrons whose energies are so low that they cannot reach the nearest boundary. Those methods are, however, inefficient for simulations involving curved surfaces, ${ }^{57}$ where the time required to calculate the distance to the closest boundary may be considerable. An alternative way is to use a range-related "region rejection" technique. In this method, different energy cutoffs are chosen for the regions surrounding the region where energy deposition is to be scored, each energy cut-off being chosen according to the distance to the nearest boundary of the region of interest.

Parameter reduced electron step. This algorithm allows us to use small electron steps in the vicinity of interfaces and boundaries and large steps elsewhere. ${ }^{53}$ Its components are the following: a path-length correction algorithm which is based on the multiple scattering theory of Moliere and which takes into account the differences between the straight pathlength and the total curved pathlength for each electron step; a lateral correlation algorithm which takes into account lateral transport; and a boundary crossing algorithm which ensures that electrons are transported accurately in the vicinity of interfaces. The algorithm has been implemented in the EGS4 code system and proved that substantial savings in computing time may be realized when using this method.

\section{General methods}

Correlated sampling. The correlated sampling technique can be used in the transport of both photons and electrons. It is especially effective for calculating ratios or differences of two quantities which are nearly equal. The basic idea is that the simulations of the geometries of interest are kept as closely correlated as possible so that most of the statistical fluctuations will cancel in the ratios and differences. The real difference between the two geometries will be better reflected in the ratios and the differences obtained. The calculational uncertainties in the ratios and the differences obtained with correlated sampling are, in general, smaller than those obtained from uncorrelated simulations.

There are several ways of doing correlated sampling in radiation transport. In coupled photon-electron transport, a simple method has been used in which random number seeds of the particle histories, for which a primary particle or any of the secondaries has deposited energy in the region of interest for one geometry, is stored and used for the simulations of the alternative geometry. ${ }^{57}$ A new correlated sampling method for the transport of electrons and photons has been developed in which a main particle history is split up whenever a particle meets the boundary of the region where the medium differs between the two or more cases. ${ }^{61}$ This particle is then followed separately for each case until it and all its descendants terminate. Holmes ${ }^{62}$ described a correlated sampling technique which forces histories to have the same energy, position, direction and random number seed as incident on both a heterogeneous and homogeneous water phantom. This ensures that a history that has, by chance, traveled through only water in the heterogeneous phantom will have the same path as it would have through the homogeneous phantom, resulting in a reduced variance when a ratio of the heterogeneous dose to the homogeneous dose is formed.

Use of geometry symmetry. The use of some of the inherent symmetry of the geometry may realize a considerable increase in efficiency. If both the source and target configu- 
rations contain cylindrical planar or spherical-conical simulation geometries, the use of symmetries is more obvious. Other uses of symmetry are less obvious, but the saving in computing time is worth the extra care and coding.

\section{NUCLEAR MEDICAL IMAGING TECHNIQUES}

Recent advances in detector design focus on enhanced sensitivity and spatial and temporal resolution, and on the possibility of using conventional photon and coincidence detection (two back-to-back photons, each with an energy of $511 \mathrm{keV}$ ) simultaneously. In this section, we describe instrumentation advances in nuclear medical imaging.

\section{A. Planar gamma camera imaging}

Gamma camera imaging requires the collimation of gamma rays emitted by the radiopharmaceutical distribution within the body. Collimators are typically made of lead or tungsten and are about 4 to $5 \mathrm{~cm}$ thick and 20 by $40 \mathrm{~cm}$ on a side. The collimator contains thousands of squares, round or hexagonal parallel channels through which gamma rays are allowed to pass. Although quite heavy, these collimators are placed directly on top of a very delicate single crystal of $\mathrm{NaI}(\mathrm{Tl})$. Any gamma camera so equipped with a collimator is called an Anger camera. ${ }^{63}$ Gamma rays traveling along a path that coincides with one of the collimator channels will pass through the collimator unabsorbed and interact with the $\mathrm{NaI}(\mathrm{Tl})$ crystal creating light. Behind the crystal, a grid of light sensitive photomultiplier tubes collect the light for processing. It is from an analysis of these light signals that images are produced. Depending on the size of the Anger camera, whole organs such as the heart and liver can be imaged. Large Anger cameras are capable of imaging the entire body and are used, for example, for bone scans.

A typical Anger camera equipped with a low-energy collimator detects roughly one in every ten thousand gamma ray photons emitted by the source in the absence of attenuation. This number depends on the type of collimator used. The system spatial resolution also depends on the type of collimator and the intrinsic resolution of the Anger camera. A typical modern Anger camera has an intrinsic resolution of 3 to 9 millimeters. Independent of the collimator, system resolution cannot get any better than intrinsic resolution. The same ideas also apply to sensitivity: system sensitivity is always worse than intrinsic (crystal) sensitivity. A collimator with thousands of straight parallel lead channels is called a parallel-hole collimator, and has a geometric or collimator resolution that increases with the distance from the gamma ray source. The geometric sensitivity, however, is inversely related to geometric resolution, which means improving collimator resolution decreases collimator sensitivity, and vice versa. High resolution and great sensitivity are two paramount goals of gamma camera imaging. Therefore, researchers must always consider this trade-off when working on new collimator designs. There have been several collimator designs in the past fifteen years, which optimized the resolution/sensitivity inverse relation for their particular design. ${ }^{64}$ Converging hole collimators, for example, fan- beam and cone-beam ${ }^{65}$ have been built to improve the tradeoff between resolution and sensitivity by increasing the amount of the Anger camera that is exposed to the radionuclide source. This increases the number of counts, which improves sensitivity. More modern collimator designs, such as half-cone-beam and astigmatic, have also been conceived. Sensitivity has seen an overall improvement by the introduction of multi-camera SPECT systems. A typical triplecamera SPECT system equipped with ultra-high resolution parallel-hole collimators can achieve a resolution of from 4 to 7 millimeters. ${ }^{2}$ Other types of collimators with only one or a few channels, called pinhole collimators, have been designed to image small organs and human extremities, such as the wrist and thyroid gland, in addition to research animals such as rats. ${ }^{66,67}$

\section{B. Single-photon emission computed tomography}

SPECT has, in recent years, become one of the major tools for the in vivo localization of radiopharmaceuticals in nuclear medicine studies. SPECT systems are now widely available and important clinical areas of SPECT imaging include cardiology, neurology, psychiatry and oncology. In conjunction with new and existing radiopharmaceuticals, quantitative SPECT may be used to noninvasively measure blood-flow, metabolic function, receptor density and drug delivery. In oncology, it is important in radiation dosimetry and treatment planning for internal radionuclide therapy in general and radioimmunotherapy (RIT), in particular. $^{2}$ Transverse tomographic images can be reconstructed from projection data acquired at discrete angles around the object. Many mathematical approaches have been used for image reconstruction in SPECT. Two broad categories have emerged, which we refer to as analytic and iterative algorithms. The common characteristic of analytic methods is that they utilize exact formulas for the reconstructed image density. The most popular method is filtered backprojection where the acquired projection data are filtered with a ramp filter before being backprojected. The iterative approach is based on the process of matching the measured projections to the calculated projections. The calculated projections are determined from an initial reconstruction and are compared to the measured data. The difference between the two data sets is used to correct the calculated projections. This procedure is repeated until some predefined error level has been reached. Statistical reconstruction techniques such as the maximum-likelihood expectation-maximization (ML-EM) algorithm seek a source distribution which will maximize the ML function relating the estimated and the measured projections.

The quantitative determination of the radioactivity content in tissues is required in both diagnostic and therapeutic nuclear medicine. Planar scintillation camera imaging has been used to estimate activity in tumors and various organs. ${ }^{68}$ The drawback with this technique is, however, the lack of information regarding the variation of activity with depth. The acquired images are, furthermore, distorted by the activity content in overlapping structures. In contrast, 
SPECT has great potential for the quantitation of activity distributions in vivo due to its three-dimensional imaging capability. There are, however, several factors that must be considered in quantitative imaging. Some of these factors are the system sensitivity and spatial resolution, dead-time and pulse pile-up effects, the linear and angular sampling intervals of the projections, the choice of reconstruction filter and the size of the object and attenuation and scatter. ${ }^{1}$ Since image quality in nuclear medicine is limited by statistics, the administered dose and the imaging time are extremely important. In practice, the limited count statistics in most clinical studies affect the accuracy and precision of quantitative SPECT. However, the two most significant effects are the photon attenuation in the object and the contribution in the images of events arising from photons scattered in the object. These effects limit the accuracy of quantitative measurements and result in decreased contrast and blurred edges of the reconstructed activity distribution in the image. ${ }^{2}$

\section{Positron emission tomography}

Measurement of the tissue concentration of a positronemitting radionuclide is based on coincidence detection of the two photons arising from positron annihilation. Following the administration of a positron-emitting radioisotope, detector arrays surrounding the patient detect the emerging annihilation photons. After being sorted into parallel projections, the lines of response (LORs) defined by the coincidence channels are used to reconstruct the three-dimensional (3D) distribution of the positron-emitter tracer within the patient. In two-dimensional (2D) PET, each 2D transverse section of the tracer distribution is reconstructed independently of adjacent sections. In fully three-dimensional (3D) PET, the data are sorted into sets of LORs, where each set is parallel to a particular direction, and is therefore a 2D parallel projection of the 3D tracer distribution. Coincidences are collected for each LOR and stored in a 2D array, or sinogram. In each sinogram, there is one row containing the LORs for a particular azimuthal angle; each such row corresponds to a 1D parallel projection of the tracer distribution at a different coordinate along the scanner axis. An event is registered if both crystals detect an annihilation photon within a coincidence time window of the order of $10 \mathrm{~ns}$, depending on the timing properties of the scintillator. A pair of detectors is sensitive only to events occurring in the tube joining the two detectors, thereby registering direction information (electronic collimation). Coincidence detection offers significant advantages over single-photon detection: electronic collimation eliminates the need for physical collimation, thereby significantly increasing sensitivity. Accurate corrections can be made for the self-absorption of photons within the patient so that absolute measurements of tissue tracer concentration can be made.

While the physics of positron annihilation limits the spatial resolution to, at best, $2-3 \mathrm{~mm}$, the statistical accuracy is related to the sensitivity of the detection system. In the past twenty years, there has been a significant evolution in PET instrumentation from a single ring of bismuth germanate (a)

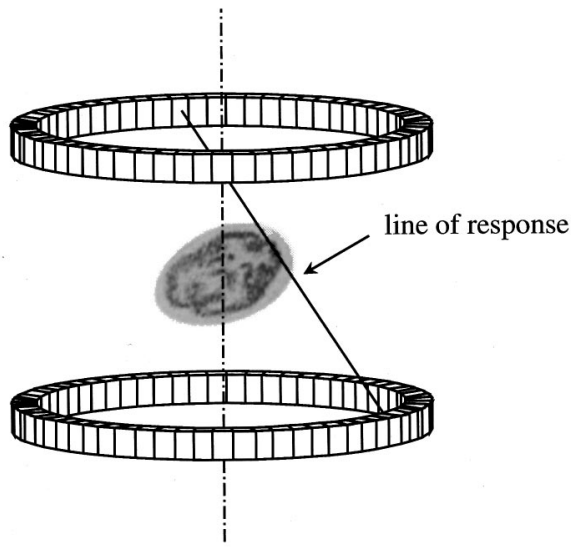

(b)

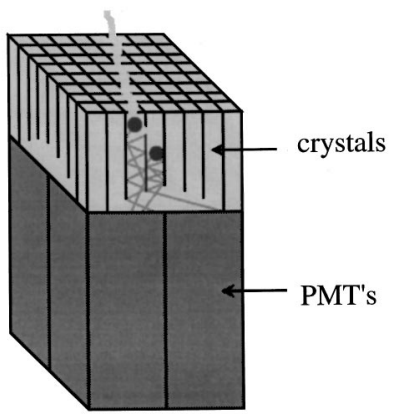

FIG. 4. (a) Schematic representation of a volume-imaging multi-ring PET scanner. (b) A block detector consists of a set of crystals having cuts of different depths acting as light guides and segmenting the block into 64 $(8 \times 8)$ detection elements in this example. The block is coupled to four photomultiplier tubes at the back, and the crystal in which photoabsorption occurs is identified by comparing the outputs of the four photomultiplier tubes.

(BGO) detectors with a spatial resolution of $15 \mathrm{~mm},{ }^{19}$ to multiple rings of small BGO crystals offering a spatial resolution of $5 \mathrm{~mm} .{ }^{69}$ The spatial resolution improvements have been achieved through smaller crystals and the efficient use of photomultipliers and position readout based on Anger logic. The tomograph design which has proved successful in recent years represents a compromise between maximizing sensitivity while keeping detector dead time and contamination from scattered and random coincidences at a reasonable level. To achieve this performance, multi-ring tomographs incorporate collimators (or septa) between the detector rings, with coincidences acquired only within a ring or between adjacent rings. ${ }^{70}$ Thus, in the interest of maximizing the signal-to-noise ratio and quantitative accuracy, comparatively little use has been made of electronic collimation, one of the main advantages of coincidence counting. Consequently, an increase in sensitivity by a factor of $4-5$ has been achieved by removing the septa and acquiring coincidences between detectors in any two rings ${ }^{71}$ (Fig. 4). It is also found that tomographs without septa can be operated more effectively with lower activity levels in the field-of-view.

A modern tomograph with inter-ring septa detects and records only $0.5 \%$ of the photon pairs emitted from the activity within the tomograph field-of-view. This increases to 
over $3 \%$ when the septa are removed. ${ }^{69}$ However, even if the detector system is $100 \%$ efficient for the detection of annihilation photons, the angular acceptance of modern scanners would record only $4.5 \%$ of the coincidences. The spatial resolution obtained with modern tomographs is about 5-6 $\mathrm{mm}$ in all three directions. Most use detectors based on $5 \mathrm{~cm} \times 5 \mathrm{~cm}$ blocks of BGO. Each BGO block is cut into 8 by 8 individual detector cells and read out by four photomultiplier tubes. Light sharing schemes are used to identify the active detector cell. Energy resolution at $511 \mathrm{keV}$ of such $\mathrm{BGO}$ detector blocks is decreased from an intrinsic value of about $15 \%$ FWHM to around $23 \%$ up to $44 \%$, depending on the cell, because of scintillation light losses resulting from the cuts applied to the detector block.

Compton scatter in the field-of-view is another effect influencing sensitivity and represents more than $30 \%$ of the data acquired with a 3D scanner. ${ }^{70}$ Increasingly sophisticated scatter correction procedures are under investigation, particularly those based on accurate scatter models, and on subtraction-convolution approaches. ${ }^{72,73}$ Monte Carlo methods give further insight and might in themselves offer a possible correction procedure. The development of fully $3 \mathrm{D}$ reconstruction algorithms has been necessary in order to take advantage of the acquisition of PET data without septa. ${ }^{74-76}$ In the most widely used 3D filtered backprojection (FBP) algorithm of Kinahan and Rogers, ${ }^{75}$ unmeasured oblique projection data, not accessible within the finite axial extension of the scanner, are estimated by forward-projecting through a low-statistics image reconstructed by 2D-FBP from transaxial projections. The completed 2D projections are then reconstructed by the FBP technique: each $2 \mathrm{D}$ projection is convolved with a 2D filter kernel, and then backprojected in $3 \mathrm{D}$ through the image volume.

\section{Multiple emission tomography}

In recent years, there has been an increased interest in using conventional SPECT scintillation cameras for PET imaging, however, the count rate performance is a limiting factor. A sandwich-like construction of two different crystals allows the simultaneous use of gamma and positron radiopharmaceuticals referred to as multiple emission tomography (MET). ${ }^{77}$ This may be implemented with solid-state photodiode readouts, which also allows electronically collimated coincidence counting (Fig. 5). The resultant images will provide finer imaging resolution (less than $5 \mathrm{~mm}$ ), better contrast and a ten-fold improvement in coincidence sensitivity when compared to what is currently available. Although the photodiode noise might be a major problem, this can be solved to some extent but with a significant increase in cost.

The performance of a detector block design which would have high resolution and high count rate capabilities in both detection modes was recently evaluated. ${ }^{23}$ The high light output of LSO (approximately 5-6 times BGO) allows the construction of a detector block that would have similar intrinsic resolution characteristics at $140 \mathrm{keV}$ as a conventional high resolution $\mathrm{BGO}$ block detector at $511 \mathrm{keV}$. However, the intrinsic radioactivity of LSO prevents the use of this

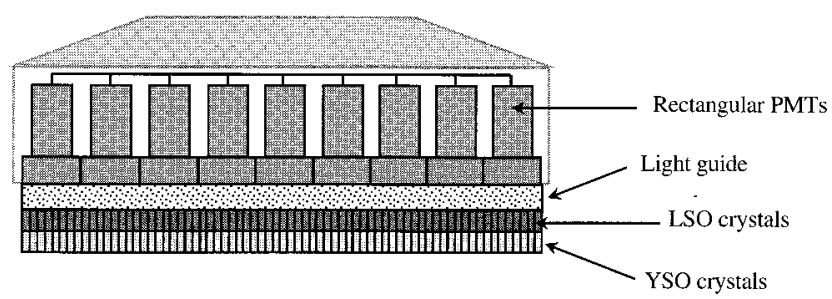

FIG. 5. The possible detector design of a multiple emission tomography camera. The detector blocks employ two separate crystals: one for singlephoton emitters (yttrium oxyorthosilicate, YSO) and one for positron emitters (lutenium oxyorthosilicate, LSO). Rectangular photomultiplier tubes (PMTs) are preferred because they reduce the dead spaces between the PMTs when compared to those of the circular ones.

scintillator in a single-photon counting mode. YSO is a scintillator with higher light output than LSO but worse absorption characteristics than LSO. YSO and LSO could be combined in a phoswich detector block, where YSO is placed in a front layer and is used for low energy SPECT imaging and LSO in a second layer is used for PET imaging. ${ }^{23}$ Events in the two detector materials can be separated by pulse shape discrimination, since the decay times of the light in YSO and LSO are different (70 and $40 \mathrm{~ns}$, respectively).

\section{APPLICATIONS OF THE MONTE CARLO METHOD IN NUCLEAR MEDICAL IMAGING}

\section{A. Detector modeling}

Monte Carlo simulation of detector responses and efficiencies is one of the areas which has received considerable attention. ${ }^{5-10}$ The critical component of emission tomography is the scintillation detector. Increased light per gamma ray interaction, faster rise and decay times, greater stopping power and improved energy resolution are the desired characteristics. Table I summarizes these properties for selected scintillators under development and currently in use. Improvements in these characteristics enable detectors to be divided into smaller elements, thus increasing resolution and minimizing dead-time losses.

An early contribution to the field providing a detailed description of the techniques used was due to Zerby. ${ }^{78}$ Tabulations of the response of $\mathrm{NaI}(\mathrm{Tl})$ detectors were performed between $100 \mathrm{keV}$ and $20 \mathrm{MeV},{ }^{79}$ and the simulations of incident photons above $300 \mathrm{keV}$ impinging on cylindrical detectors of different materials due to Rogers. ${ }^{80}$ Simulations of $\mathrm{NaI}(\mathrm{Tl})$ detectors with different shapes and volumes below $300 \mathrm{keV}$ have also been reported. ${ }^{81}$ A detailed investigation of energy responses of germanium detectors and the use of Monte Carlo simulations to correct the measured spectra has been performed by $\mathrm{Chan}^{82}$ and comparisons of efficiency calculations for BGO scintillators between Monte Carlo and measurements reported. ${ }^{83}$ The detection efficiency of a highpressure, gas scintillation proportional chamber, designed for medical imaging in the 30-150 keV energy range, has been investigated through measurement and Monte Carlo simulation with the aim to design an optimized detector for use in specialized nuclear medicine studies. ${ }^{84}$ An approximate ex- 
TABLE I. Characteristics of scintillator crystals under development and currently used in nuclear medicine imaging systems.

\begin{tabular}{|c|c|c|c|c|c|c|c|}
\hline Scintillator & $\mathrm{NaI}(\mathrm{Tl})$ & BGO & $\mathrm{BaF} 2$ & LSO & GSO & LuAP & YAP \\
\hline Formula & $\mathrm{NaI}(\mathrm{Tl})$ & $\mathrm{Bi}_{4} \mathrm{Ge}_{3} \mathrm{O}_{12}$ & $\mathrm{BaF}_{2}$ & $\mathrm{Lu}_{2} \mathrm{SiO}_{5}: \mathrm{Ce}$ & $\mathrm{Gd}_{2} \mathrm{SiO}_{5}: \mathrm{Ce}$ & $\mathrm{LuAlO}_{3}: \mathrm{Ce}$ & $\mathrm{YAlO}_{3}: \mathrm{Ce}$ \\
\hline Density (g/cc) & 3.67 & 7.13 & 4.89 & 7.4 & 6.71 & 8.34 & 5.37 \\
\hline Light yield (\%) & 100 & $15-20$ & $3-20$ & 75 & $20-25$ & $25-50$ & 40 \\
\hline Effective $Z$ & 51 & 75 & 53 & 66 & 60 & 65 & 34 \\
\hline Decay constant (ns) & 230 & 300 & $1 / 700$ & 42 & $30-60$ & 18 & 25 \\
\hline Peak wavelength (nm) & 410 & 480 & $195-220$ & 420 & 440 & 370 & 370 \\
\hline index of refraction & 1.85 & 2.15 & 1.56 & 1.82 & 1.95 & 1.95 & 1.56 \\
\hline Photofraction $(\%)^{\mathrm{a} / \mathrm{b}}$ & $17.3 / 7.7$ & $41.5 / 88$ & $18.7 / 78.6$ & $32.5 / 85.9$ & $25 / 82.3$ & $30.6 / 85.1$ & $4.5 / 48.3$ \\
\hline Mean free path $(\mathrm{cm})^{\mathrm{a} / \mathrm{b}}$ & $2.93 / 0.4$ & $1.04 / 0.08$ & $2.19 / 0.27$ & $1.15 / 0.1$ & $1.4 / 0.16$ & $1.05 / 0.1$ & $2.17 / 0.7$ \\
\hline Hygroscopic & Yes & No & No & No & No & No & No \\
\hline
\end{tabular}

${ }^{\mathrm{a} A t} 511 \mathrm{keV}$.

${ }^{\mathrm{b}}$ At $140 \mathrm{keV}$.

pression for the count rate characteristics of Anger scintillation cameras has been derived, and validated by Monte Carlo simulations ${ }^{85}$ while the EGS4 Monte Carlo code has been used to evaluate the response of $\mathrm{HgI}_{2}$ crystal in terms of efficiency, energy and space resolutions versus photon energy in the diagnostic energy range $(20-100 \mathrm{keV}) .^{86}$

Many detector modeling applications were developed in the PET field, including the pioneering work of Derenzo, ${ }^{87}$ who simulated arrays of detectors of different materials and sizes to study the effect of the inter-crystal septa and later on to optimize the optical coupling between BGO crystals and PMTs $^{88}$ by taking into account the reflection and scattering along the detection system. The search for an appropriate detector for this imaging modality was conducted in a comparative study of several crystals including BGO, CsF and $\mathrm{NaI}(\mathrm{Tl}),{ }^{89} \mathrm{BaF}_{2}$ used in time-of-flight PET, ${ }^{90}$ and liquid Xenon. ${ }^{91}$ Binkley ${ }^{92}$ modeled the impulse response of a PMT, front-end amplifier, and constant fraction discriminator to evaluate the effects of front-end bandwidth and constant fraction delay and fraction for timing-system optimizations of BGO scintillation detectors.

The penetration of annihilation photons into the detector material before interaction is a statistical process which leads to significant displacement and anisotropy of the point spread function. Compensation for crystal penetration is thus an important issue to recover the spatial resolution in PET. ${ }^{93}$ Comanor $^{94}$ investigated algorithms to identify and correct for detector Compton scatter in hypothetical PET modules with $3 \times 3 \times 30 \mathrm{mmBGO}$ crystals coupled to individual photosensors. The true crystal of first interaction was determined by the simulation for eventual comparison with the crystal identified by a given algorithm. They reported a misidentification fraction of $12 \%$ if the detector has good energy and position resolution when using position of interaction to identify forward scatter.

Numerous strategies have been proposed for constructing detector modules that measure the depth of interaction (DOI), but most of them proved impractical to implement or provided insufficient DOI measurement resolution. Two important questions can be addressed through Monte Carlo simulation: (i) what fraction of events will be mis-identified because of noise fluctuations in the photomultiplier tubes
(PMT's) or photodiode array and (ii) how will the DOI measurement resolution affect the reconstructed resolution of a PET camera? The position-dependent light distribution has been used to measure the $511 \mathrm{keV}$ photon interaction position in the crystal on an event by event basis to reduce radial elongation. ${ }^{95}$ Different geometrical modifications were also simulated, leading to a proposal of a $2.2 \times 5 \times 30 \mathrm{mmBGO}$ crystal, for which a $2.2 \mathrm{~mm}$ FWHM light distribution is predicted, which should yield a PET detector module with DOI measurement resolution of $3.6 \mathrm{~mm}$ FWHM. A test module with one $3 \times 3 \times 30 \mathrm{mmBGO}$ crystal, one $3 \mathrm{~mm}$ square PIN photodiode and one PMT operated at $-20^{\circ} \mathrm{C}$ with an amplifier peaking time of $4 \mu \mathrm{s}$, and a measured DOI resolution of 5 to $8 \mathrm{~mm}$ FWHM has been proposed by Moses. ${ }^{96}$ Simulations predicted that this virtually eliminates radial elongation in a $60 \mathrm{~cm}$ diameter $\mathrm{BGO}$ tomograph. The performance of a single detector element must be extrapolated using Monte Carlo simulations to predict the performance of a multielement module or a complete PET camera.

The Triumph PET group has developed a simulation tool to model position encoding multicrystal detectors for PET that treats the interactions of energetic photons in a scintillator, the geometry of the multi-crystal array, as well as the propagation and detection of individual scintillation photons. ${ }^{97}$ Design studies of a whole-body PET tomograph with the capacity to correct for the parallax error induced by the DOI of gamma-rays were also performed. ${ }^{98}$ The experimental energy, depth and transverse position resolutions of BGO block detectors were used as main inputs to the simulations to avoid extensive light transport in position encoding blocks. An improved model for energy resolution which includes the nonproportionality of the scintillation response of BGO and the statistical noise from photoelectron amplification in the PMT's was also proposed. ${ }^{99}$ Simulation studies have also been carried out to investigate the feasibility of using a triangular detection module for PET with neutral networks to reconstruct the coordinates of the photon absorption point and thus recover the DOI information. ${ }^{100}$ Another exciting application is the use of a PET imaging system for monitoring the dose delivered by proton and gamma-ray radiotherapy beams. ${ }^{101}$ By measuring the amount and ratio of 
the beam-induced positron-emitting activity, the dose distribution and tissue composition may be determined.

\section{B. Imaging systems and collimators design}

Image modeling was employed by Schulz, ${ }^{102}$ who devised a computer program simulating a rectilinear scanner which was used to study the influence of different imaging protocols on the detectability of lesions. Simulation of gamma camera imaging to assess qualitatively and quantitatively the image formation process and interpretation ${ }^{103}$ and to assist development of collimators ${ }^{104}$ using deterministic methods and simplifying approximations have been developed mainly to improve speed of operation.

In gamma camera imaging, the choice of collimator involves a compromise between sensitivity and spatial resolution. ${ }^{64,65}$ The proper choice of collimator is especially difficult at the cut-off energy level of low-energy collimators (e.g., $\left.{ }^{123} \mathrm{I} ; 159 \mathrm{keV}\right)$ and in multiple tracer studies. The relationships between sensitivity, spatial resolution and septal penetration of a given set of collimators have to be studied. ${ }^{1,105}$ The physicist has to determine which of the available collimators provides superior image quality for a given acquisition time. ${ }^{106}$ To that end, in addition to its quantitative clinical applications, Monte Carlo simulation may be a useful research tool for tasks such as evaluating collimator design and optimizing gamma camera motion. In recent years, there is an increased use of specialized collimators such as fan-beam, ${ }^{65}$ convergent-beam, ${ }^{64}$ concave, ${ }^{107}$ variable focus (cardiofocal) and long-bore collimators. The improvement in image quality results from the fact that the increase in resolution is greater than the loss of sensitivity. The effect of collimation in a Compton-scatter tissue densitometry scanner has been studied in a detailed paper. ${ }^{108}$

Monte Carlo techniques were extensively used to analyze the performance of new collimators design for planar gamma camera, ${ }^{109,110}$ SPECT ${ }^{111}$ and PET imaging. ${ }^{112,113}$ Practical guidance could be offered for understanding trade-offs that must be considered for clinical imaging. Selective comparisons among different collimators could also be presented for illustrative and teaching purposes. Approaches to the collimator optimization problem, as well as more sophisticated "task-dependent" treatments and important considerations for collimators design have been performed. ${ }^{114}$ The wellknown imaging performance parameters of parallel-hole collimators could be compared with those of fan- and conebeam collimators ${ }^{106,115}$ which have enjoyed considerable success in recent years, particularly for brain SPECT. Reduced noise and higher sensitivity was reported for conebeam collimators compared to other collimators having similar geometric resolutions. Webb ${ }^{109}$ proposed a rotating-slit collimator which collects one-dimensional projections from which the planar image may be reconstructed by the theory of computed tomography. A spatial resolution of $6 \mathrm{~mm}$ at a distance of $100 \mathrm{~mm}$ from the collimator with seven times the sensitivity of a parallel-hole collimator was achieved. The imaging properties of optimally designed planar-concave collimators were evaluated by means of Monte Carlo simulations. ${ }^{111}$ The authors showed that the image noise distribution along the object radius became more uniform when the curved collimator was used and that the spatial resolution of the lateral cortex when using the curved collimator was significantly improved due to improved radial resolution. Monte Carlo calculations were also used to aid in the development of a method for imaging therapeutic doses of ${ }^{131} \mathrm{I}$ by using thick $\mathrm{Pb}$ sheets to the front face of a high-energy parallel-hole collimator. ${ }^{116}$

There has been renewed interest in pinhole collimation for high resolution imaging of small organs such as the thyroid since it provides an improved spatial resolution and an increase in sensitivity as the distance between the source and the pinhole aperture decreases. ${ }^{67}$ Wang ${ }^{117}$ simulated point response functions for pinhole apertures with various aperture span angle, hole size and materials. The point responses were parameterized using radially circularly symmetric twodimensional exponential functions which can be incorporated into image reconstruction algorithms that compensate for the penetration effect. The effect of pinhole aperture design parameters on angle-dependent sensitivity for high resolution pinhole imaging was also investigated using Monte Carlo modeling. ${ }^{118}$ Simulated ${ }^{131}$ I SPECT studies for uniform cylinders showed that activity concentrations were underestimated toward the outside of the cylinders when a $\sin ^{3} \theta$ rather than the correct $\sin ^{x} \theta$ sensitivity correction was applied in image reconstruction, where $x$ is a parameter and $\theta$ is the angle of the incident ray with the surface of the detector crystal.

In a similar way in the PET field, Monte Carlo techniques were used to determine the effects of crystals with straight and pointed tips and septa on spatial resolution and efficiency, ${ }^{119}$ to compare the singles to true coincident events ratios in well collimated single, multi-slice and open collimator 3D configurations, ${ }^{112}$ to evaluate tungsten inter-plane septa of different thicknesses and geometries ${ }^{113}$ and to assess the effect of collimation on the scatter fraction. ${ }^{120}$

The design of SPECT and PET systems using the Monte Carlo method has received considerable attention and a large number of applications were the result of such investigations. ${ }^{121,122}$ Bradshaw $^{121}$ used this tool for the design of a detector suitable for use in a SPECT cylindrically shaped scintillation camera. Detection characteristics of two scintillator materials and the optical performance of several geometric configurations were studied. The design of prototype systems that utilize solid-state detectors and low-noise electronics to achieve improved energy resolution were carried out using simulated SPECT projections of a simple myocardial perfusion phantom. ${ }^{123}$ The results showed that a FWHM energy resolution of 3-4 keV is sufficient to render the error due to scatter insignificant compared to the uncertainty due to photon statistics. Monte Carlo simulations have also been performed to evaluate the design of collimated detectors used to measure ${ }^{125} \mathrm{I}$ or ${ }^{131} \mathrm{I}$ in the thyroid gland. ${ }^{124}$ Two detector sizes were simulated for each radioisotope and activity was placed in both the gland and the remainder of the body in varying amounts to assess the efficacy of collimation. This study showed that a wide angle of acceptance 
and sufficient detector crystal thickness take precedence over collimation and shielding.

The Monte Carlo method has also been used in the design of single-slice ${ }^{19,122}$ and multi-slice PET scanners. ${ }^{70,71} \mathrm{~A}$ VME bus-based microcomputer system has been used to implement a model for simulation of the flux of gamma rays in cylindrical PET detector systems. ${ }^{125}$ The program is capable of tracing over one million photons per hour and has been used to explore some of the effects of "opening up" planar detector geometries into volumetric imagers. Rogers ${ }^{126}$ compared some of the performance parameters of a tomograph based on large area $\mathrm{NaI}(\mathrm{Tl})$ detectors to similar parameters of conventional small crystal machines. Michel ${ }^{70}$ used the GEANT package from CERN ${ }^{43}$ to study the response function and the scatter fraction in two PET scanners with and without inter-plane septa. The simulation of a large multi-plane PET camera named HISPET ${ }^{127}$ and a planar imaging system made of two matrices, each one consisting of $400\left(2 \times 2 \times 30 \mathrm{~mm}^{3}\right)$ crystals of YAP:Ce ${ }^{128}$ using the EGS4 system have also been reported. Thompson ${ }^{129}$ investigated the effects of detector material and structure on PET spatial resolution and efficiency in terms of the number of interactions and tangential component of the mean square distance between the centroid and the point of first interaction.

Several researchers used Monte Carlo simulation methods to study potential designs of dedicated small animal positron tomographs. ${ }^{130,131}$ An important conclusion drawn from these studies is that unlike human imaging where both sensitivity and spatial resolution limitations significantly affect the quantitative imaging performance of a tomograph, the imaging performance of dedicated animal tomographs is almost solely based upon its spatial resolution limitations. ${ }^{132}$ Recently, a conceptual design for a PET camera designed to image the human brain and small animals has been presented. ${ }^{133}$ The authors performed a Monte Carlo simulation to predict the spatial resolution for a single plane PET camera with $3 \mathrm{~mm}$ LSO crystals. They concluded that the detector modules must be able to measure the DOI on an event by event basis in order to eliminate radial elongation artifacts, and that such depth information can be incorporated into the reconstruction algorithm in an artifact free way with a simple rebinning method.

\section{Image reconstruction algorithms}

Monte Carlo simulations have been shown to be very useful for validation and comparative evaluation of image reconstruction techniques since it is possible to obtain a reference image to which reconstructed images should be compared. Three different algorithms for performing PET image reconstruction have been compared using Monte Carlo phantom simulations. ${ }^{134}$ The results demonstrate the importance of developing a complete 3D reconstruction algorithm to deal with the increased gamma detection solid angle and the increased scatter fraction that result when the interslice septa are removed from a multi-ring tomograph. The Eidolon Monte Carlo package ${ }^{135}$ was used to simulate projection data of the cold rod phantom both with and with-
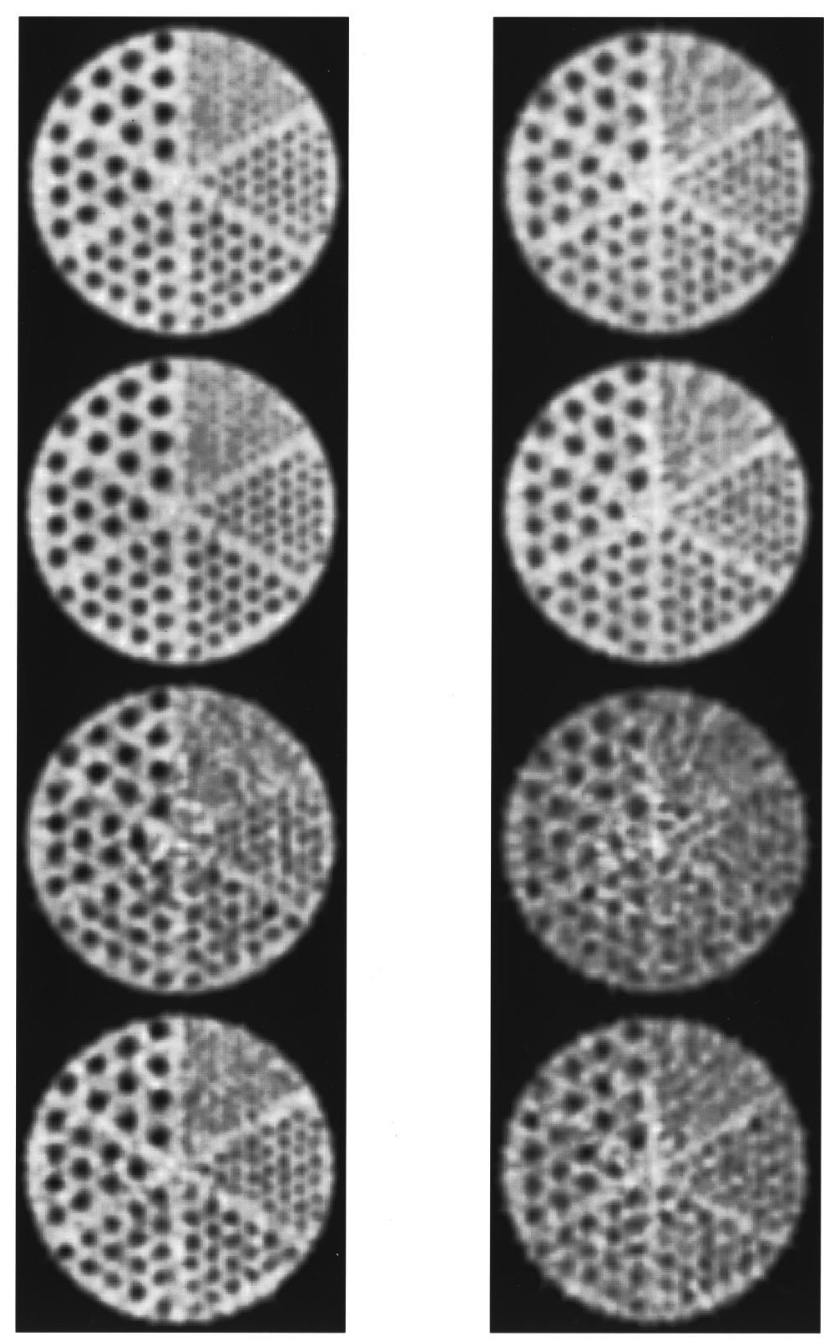

FIG. 6. Reconstructions of Monte Carlo data sets of the Jaszczack's cold rod phantom generated without (left) and with (right) scatter simulation using from top to bottom the PROMIS, FAVOR, FORE, and SSRB algorithms. The cold rod diameters are from top right counter clockwise: 4.8, 6.4, 7.9, 9.5, 11.1 and $12.7 \mathrm{~mm}$. Approximately 25 Mcounts were recorded for both types of simulations.

out scatter simulation. Figure 6 shows transaxial slices of the phantom reconstructed using four analytic algorithms: the reprojection algorithm (PROMIS) ${ }^{75}$ the fast volume reconstruction algorithm (FAVOR) ${ }^{76}$ the Fourier rebinning algorithm (FORE) ${ }^{136}$ and the single-slice rebinning algorithm (SSRB). ${ }^{74}$ Using simulated data, Hanson ${ }^{137}$ validated a method of evaluating image recovery algorithms based on the numerical computation of how well a specified visual task can be performed on the basis of the reconstructed images. Task performance was rated on the basis of the detectability index derived from the area under the receiver operating characteristic curve. Three-dimensional photon detection kernels characterize the probabilities that photons emitted by radioisotopes in different parts of the source region will be detected at particular projection pixels of the projection images. ${ }^{138}$ Smith $^{139}$ used Monte Carlo modeling to study these kernels for the case of parallel-hole collimators. The authors also proposed a reconstruction method using the 
3D kernels in which projection measurements in three adjacent planes are used simultaneously to estimate the source activity of the center plane. The matrix equations for image reconstruction are solved using generalized matrix inverses. King ${ }^{140}$ conducted a Monte Carlo study to investigate the artifacts caused by liver uptake in SPECT perfusion imaging and to verify the hypothesis that the cardiac count changes are due to the inconsistencies in the projection data input to reconstruction. A correction of the causes of these inconsistencies before reconstruction, or including knowledge of the physics underlying them in the reconstruction algorithm, would virtually eliminate these artifacts.

Floyd ${ }^{141}$ evaluated convergence properties of the ML-EM algorithm for SPECT image reconstruction as a function of Poisson noise, precision of the assumed system resolution model and iteration number. It was also shown that lesion contrasts and signal-to-noise ratios in ML-EM estimates of SPECT images can be improved by considering Compton scattering when calculating the photon detection probability matrix. ${ }^{142}$ Bayesian reconstruction methods introduce prior information, often in the form of a spatial smoothness regularizer. More elaborate forms of smoothness constraints may be used to extend the role of the prior beyond that of a stabilizer in order to capture actual spatial information about the object. ${ }^{143}$ In recent years, many investigators proposed Gibbs prior models to regularize images reconstructed from emission computed tomography data. Unfortunately, the hyperparameters used to specify Gibbs priors can greatly influence the degree of regularity imposed by such priors and, as a result, numerous procedures have been proposed to estimate hyperparameter values from observed image data. Higdon ${ }^{144}$ used recent results in Markov chain Monte Carlo sampling to estimate the relative values of Gibbs partition functions. Using these values, sampling was performed from joint posterior distributions on image scenes. This allows for a fully Bayesian procedure which does not fix the hyperparameters at some estimated or specified value, but enables uncertainty about these values to be propagated through the estimated intensities.

Maximum a posteriori (MAP) reconstruction has been shown to have significant advantages over traditional ML methods in terms of noise performance, but these advantages are highly dependent on the choice of the distribution used to model the prior knowledge about the solution image. A MAP approach for iterative reconstruction based on a weighted least-squares conjugate gradient (WLS-CG) algorithm was proposed and validated using simulated hot-sphere phantom SPECT data and patient studies. ${ }^{145}$ The ill-posed nature of tomography leads to slow convergence for standard gradientbased iterative approaches such as the steepest descent or the conjugate gradient algorithm. Chinn and Huang ${ }^{146}$ proposed a preconditioned conjugate gradient (PCG) iterative algorithm for WLS reconstruction in order to accelerate the convergence rate of iterative reconstruction. Using simulated PET data of the Hoffman brain phantom, the authors have shown that the convergence rate of PCG can reduce the number of iterations of the standard conjugate gradient algorithm by a factor of 2-8 times depending on the convergence criterion.

The search for unified reconstruction algorithms led to the development of inverse Monte Carlo (IMC) reconstruction techniques. ${ }^{147}$ The concept of IMC was introduced in 1981 in an attempt to describe a numerical method for solving a class of inverse problems. ${ }^{148}$ The IMC method converts the inverse problem, through a noniterative simulation technique, into a system of algebraic equations that can be solved by standard analytical or numerical techniques. The principal merits of IMC are that, like direct Monte Carlo, the method can be applied to complex and multivariable problems, and variance reduction procedures can be applied. The noniterative IMC is strongly related to the variance reduction technique in direct simulation called importance sampling where the sampling process is altered by using random numbers from a modified distribution. Floyd ${ }^{149}$ used IMC to perform tomographic reconstruction for SPECT with simultaneous compensation for attenuation, scatter and distance-dependent collimator resolution. A detection probability matrix is formed by Monte Carlo solution to the photon transport equation for SPECT acquisition from a unit source activity in each reconstruction source voxel. The measured projection vector $p_{j}$ will equal the product of this detection probability matrix $A_{i j}$ with the unknown source distribution vector $s_{i}$ :

$$
\left[p_{j}\right]=\left[A_{i j}\right]\left[s_{i}\right] \text {. }
$$

The resulting large, nonsparse system of equations was solved for the source distribution using an iterative ML-EM estimator. The IMC technique proved to provide compensation for the collimator effects in addition to providing higher resolution. ${ }^{150}$ It is worth noting that although the technique was developed for SPECT, it is also valid for other imaging techniques like PET and transmission CT.

The ability to theoretically model the propagation of photon noise through emission computed tomography reconstruction algorithms is crucial in evaluating the reconstructed image quality as a function of parameters of the algorithm. Wilson ${ }^{151}$ used a Monte Carlo approach to study the noise properties of the ML-EM algorithm and to test the predictions of the theory. The ML-EM statistical properties were calculated from sample averages of a large number of images with different noise realizations. The agreement between the more exact form of the theoretical formulation and the Monte Carlo formulation was better than $10 \%$ in most cases examined, and for many situations the agreement was within the expected error of the Monte Carlo experiments. The same methodology was also followed to analyze a MAP-EM algorithm incorporating an independent gamma prior, and a onestep-late (OSL) version of a MAP-EM algorithm incorporating a multivariate Gaussian prior, for which familiar smoothing priors are special cases. ${ }^{152}$

\section{Attenuation and scatter correction techniques}

The presence of scatter and attenuation in the images limits the accuracy of quantification of activity. ${ }^{1}$ With no corrections, the uncertainty could be as high as $50-100 \%{ }^{2}$ 


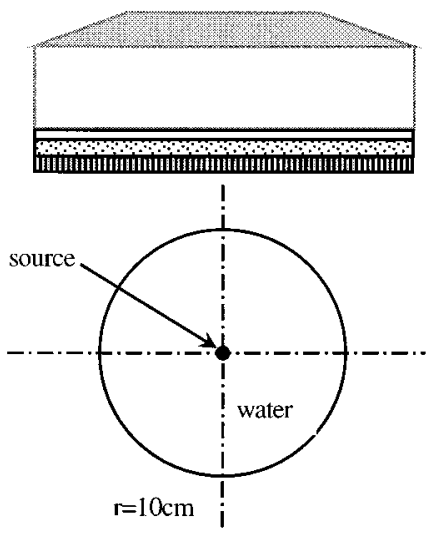

(a)

Scatter does not produce major artifacts comparable to attenuation but reduces image contrast by including a lowfrequency blur in the image. The impact of scatter generally depends on the photon energy, camera energy resolution, and energy window settings, besides the object shape and the source distribution. ${ }^{153,154}$ Many of these parameters are nonstationary which implies a potential difficulty when developing proper scatter and attenuation correction techniques. However, correction for scatter remains essential, not only for quantification, but also for lesion detection and image segmentation. ${ }^{155}$ For the latter case, if the boundary of an activity region is distorted by scatter events, then the accuracy in the calculated volume will be affected. ${ }^{156}$ Monte Carlo calculations have been found to be powerful tools to quantify and correct for photon attenuation and scattering in nuclear medicine imaging since the user has the ability to separate the detected photons into their components: primary events, scatter events, contribution of down-scatter events, etc. Monte Carlo modeling thus allows a detailed investigation of the spatial and energy distribution of Compton scatter which would be difficult to perform using present experimental techniques, even with very good energy resolution detectors. $^{157}$

In gamma camera imaging and SPECT, simulation programs have been used to obtain information on the different processes occurring within the phantom and the detectors. For example, energy pulse-height distribution, point-spread function and the scatter fraction can be obtained. ${ }^{158}$ The scattered events in the energy-pulse-height distribution can be separated according to the number of scattering events in the phantom (Fig. 7). It is clearly shown that a significant number of scattered events will be accepted by the photopeak energy window. The scatter fraction is of great importance for quantitative estimation of the scattering contribution. ${ }^{159} \mathrm{It}$ is defined as the ratio between the number of scattered photons and the total number of photons (scattered and unscattered). The scatter fraction is generally measured by scanning a line source placed at the center of a water-filled cylinder. Line spread functions (LSFs) are generated and the scatter fraction determined by fitting the scatter tails of the LSFs to a mono-exponential function (Fig. 8). The scatter fraction is calculated as scatter/total where total and scatter are calculated as the integral of the LSF and the fit within the diameter of the field-of-view. Figure 9 compares scatter fractions for different source depths and energy window sizes calculated with different Monte Carlo codes simulating scintillation camera characteristics. ${ }^{13-15}$ Beekman $^{160}$ developed a fast analytic simulator of tomographic projection data taking into account attenuation, distance-dependent detector response and scatter based on an analytical point spread function (PSF) model. Several simplifying approximations were also adopted to improve the speed of operation; restriction of the extent of the primary and scatter PSFs, coarse sampling of the PSFs in the direction perpendicular to the camera face and use of a circularly symmetric scatter function. ${ }^{161}$

A study of the factors mostly responsible for spectral contamination (overlapping of unscattered and scattered events throughout the energy spectrum) including nuclear medicine imaging instrumentation itself has been performed. ${ }^{162}$ Frey $^{163}$ generated scatter response functions (SRFs) using Monte Carlo techniques and investigated the characteristics

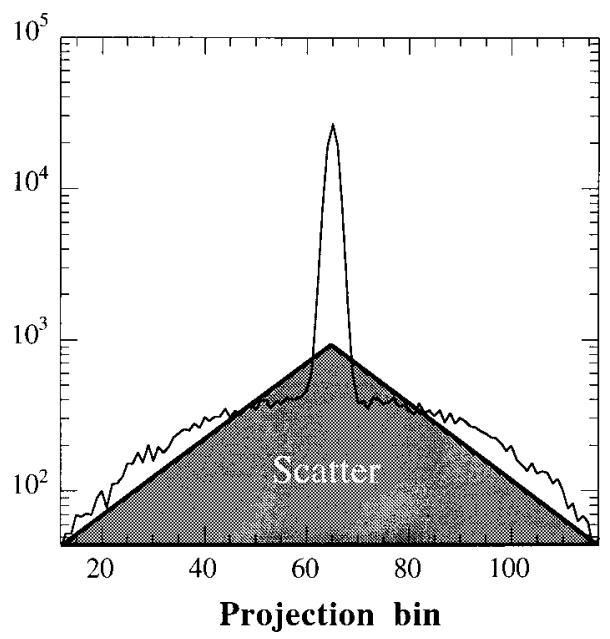

FIG. 8. Experimental determination of the scatter fraction by fitting the scatter tails to a monoexponential function. The scatter fraction is calculated as the integral of the scatter tails (gray area) to the integral of the LSF, within the diameter of the FOV. 


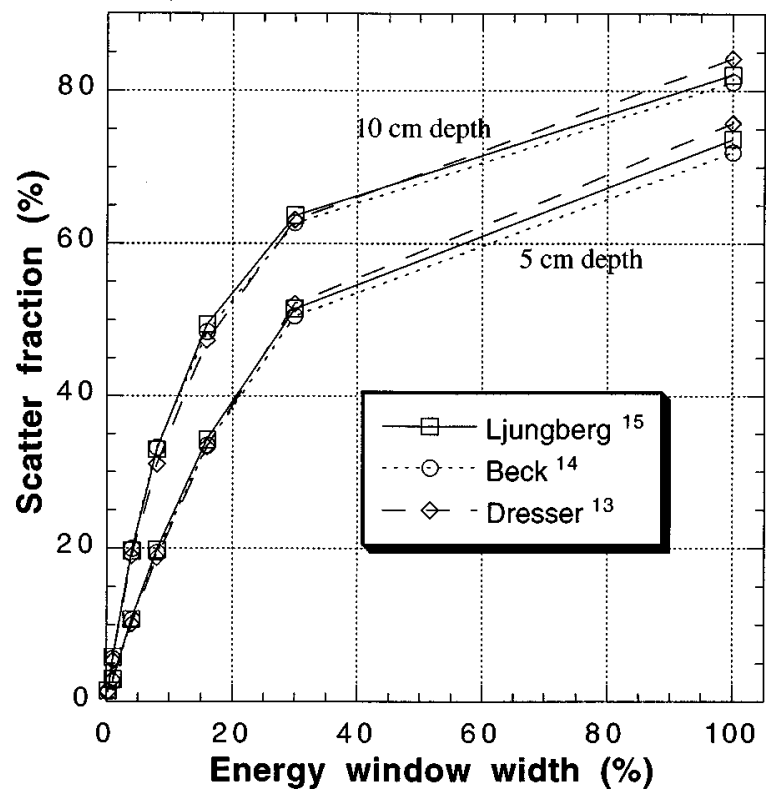

FIG. 9. A comparison of simulated scatter fractions for different source depths and energy window sizes obtained with different Monte Carlo codes simulating scintillation camera characteristics.

of the scattered radiation by fitting the SRFs with fitting functions. The parameters of the fitting functions were studied as a function of source position in a water-filled cylindrical phantom with circular cross-section. A third-order polynomial for modeling the SRF and an approximately constant fitting window was also proposed. ${ }^{164}$ SRFs were also simulated for inhomogeneous scattering media. ${ }^{165,166}$ This model has been implemented in a projector-backprojector pair that makes iterative reconstruction based scatter compensation feasible. $^{167}$

Ljungberg ${ }^{168}$ simulated both parallel and fan-beam transmission imaging to study the effect of down-scatter from an emission ${ }^{99 \mathrm{~m}} \mathrm{Tc}$ radionuclide into the energy window for a transmission ${ }^{153} \mathrm{Gd}$ radionuclide. An investigation of the effects of scattered photons in gamma-ray transmission CT for several types of data acquisition systems was also performed including a flood source and a parallel-hole collimator, a collimated flood source and a parallel-hole collimator, a line source and a symmetric fan-beam collimator and a collimated line source and a symmetric fan-beam collimator. ${ }^{169}$ The results showed that a fan-beam collimator and line source rejected most of the scattered collimated emitted photons at the object side, and that almost all the scattered photons could be rejected at the collimator on the detector side. Speller and Horrocks ${ }^{170}$ studied multiple scatter effects at lower energies, including incident diagnostic x-ray spectra, and obtained correction factors for clinical use in tissue densitometry.

Much research and development has been concentrated on the scatter compensation required for quantitative SPECT. ${ }^{1,2}$ Floyd $^{171}$ used Monte Carlo simulations to validate the basic assumptions underlying the empirical implementation of their scatter subtraction algorithm. Three scatter correction techniques for SPECT have been assessed and compared where scatter coefficients and parameters characteristic of each technique have been calculated through Monte Carlo simulations and experimental measurements for various source geometries. ${ }^{172}$ The Compton scatter correction method based on factor analysis of dynamic structures was evaluated both on planar imaging and SPECT data using Monte Carlo simulations and real phantoms. ${ }^{173}$ A comparison with the modified dual-window (DW) method was also presented. Ljungberg ${ }^{174}$ derived a method based on the combined use of $3 \mathrm{D}$ density information provided by computed tomography to correct for attenuation and the application of Monte Carlo simulated build-up factors to correct for build-up in the projection pixels. A similar method was also proposed for planar imaging. ${ }^{175}$ The effects of tissuebackground activity, tumor location, patient size, uncertainty of energy windows and definition of the tumor region on the accuracy of quantification were investigated by calculating the multiplier which yields correct activity for the volumeof-interest when using the DW method. ${ }^{176}$

A scatter correction method in which Monte Carlo simulated scatter line-spread functions (SLSF) for different depth and lateral positions has been developed. ${ }^{177}$ The uncorrected reconstructed images are convolved with the SLSF and subtracted from the projection data to yield scatter-corrected projections. The method was further validated using a clinically realistic, nonhomogeneous, computer phantom. ${ }^{178}$ Naude $^{179}$ studied the accuracy of the channel ratio scatter correction technique which is based on the assumption that the ratio of the scatter components in the two windows $(\mathrm{H}$ value) is constant and independent of the relative size of the scatter contribution. The results have shown that although the true $\mathrm{H}$ value depends on both source size and depth of the source in the scattering medium, the channel ratio technique can be applied successfully when an average $H$ value is used. Welch ${ }^{180}$ developed a method based on the use of a transmission map to define the inhomogeneous scattering object for modeling the distribution of scattered events in emission projection data. The probability of a photon being scattered through a given angle and being detected in the emission energy window was approximated using a Gaussian function whose parameters were determined using Monte Carlo generated parallel-beam SLSFs from a nonuniformly attenuating phantom. A combined scatter and attenuation correction that does not require a transmission scan was also proposed and validated using measured planar data and simulated SPECT for ${ }^{111}$ In imaging. ${ }^{181}$

Hademenos ${ }^{182}$ applied a modified dual photopeak window (DPW) scatter correction method to Monte Carlo simulated ${ }^{201} \mathrm{Tl}$ emission images. This method was also applied to two views of an extended cardiac distribution within an anthropomorphic phantom, resulting in at least a six-fold improvement between the scatter estimate and the Monte Carlo simulated true scatter. A simulation study of the triple energy window (TEW) method was conducted in a multiradionuclide $\left({ }^{99 \mathrm{~m}} \mathrm{Tc} /{ }^{201} \mathrm{Tl}\right)$ SPECT study. ${ }^{183} \mathrm{~A}$ good agreement between the activity distributions reconstructed from primary photons and those from corrected data has been shown. A spill-down correction method was also proposed 


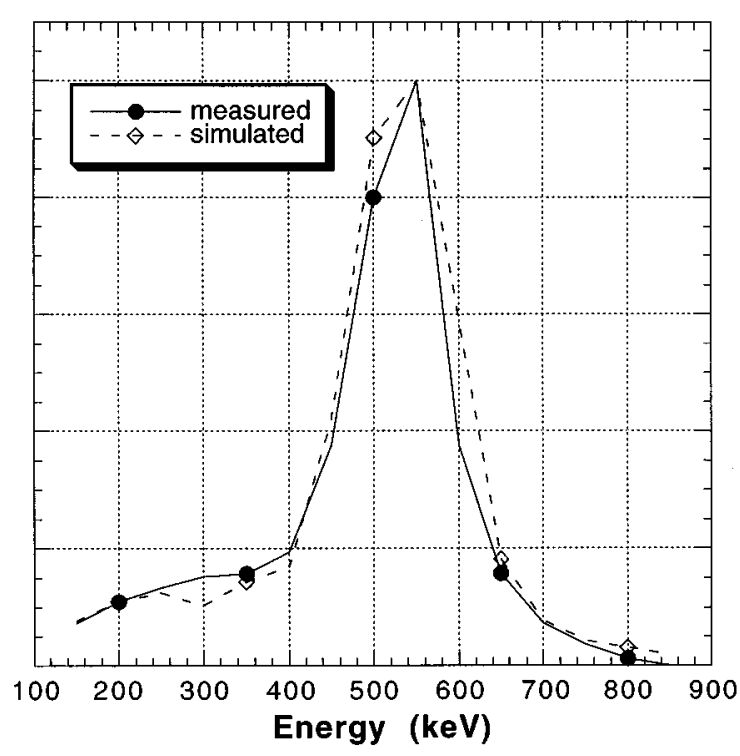

(a)

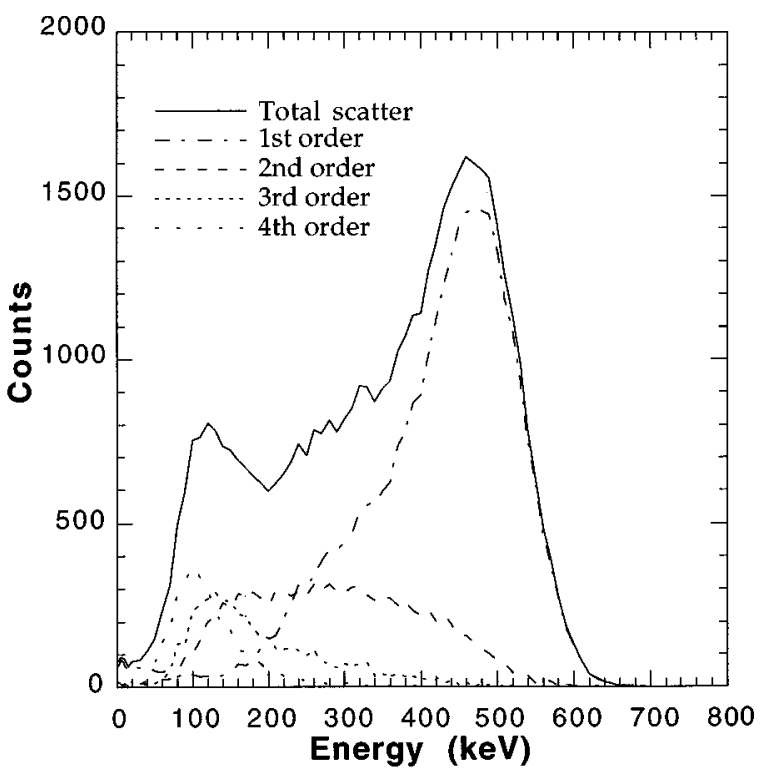

(b)

FIG. 10. (a) A comparison between measured and simulated single energy spectra of the ECAT-953B PET scanner (reprinted with permission from Ref. 191). (b) The energy distribution due to scattered photons resulting from the simulation of a line source placed in the center of a $20 \mathrm{~cm}$ diameter water-filled cylinder is separated into different contributions (total scattering or different orders of photon scattering). Energy resolution is proportional to the inverse square root of the deposited energy and is simulated by convolving the deposited energy with a Gaussian function whose FWHM is $23 \%$ for 511 keV photons.

for the ${ }^{201} \mathrm{Tl}$ window image in simultaneous dual-isotope ${ }^{99 \mathrm{~m}} \mathrm{Tc} /{ }^{201} \mathrm{Tl}$ SPECT imaging based on a single acquisition into three energy windows. ${ }^{184}$ Using Monte Carlo techniques, the fractional amount of ${ }^{99 \mathrm{~m}} \mathrm{Tc}$ and ${ }^{201} \mathrm{Tl}$ spill-down in the ${ }^{201} \mathrm{Tl}$ window with respect to the total counts from the spill-down window, was calculated for simulated images of point sources at varying depths within a water-filled elliptical tub phantom.

The DW and the convolution (CV) scatter correction techniques were compared using projection data, simulated by the Monte Carlo method. ${ }^{185}$ The scatter distributions predicted by the $\mathrm{CV}$ technique were found to be consistently lower than those simulated by the Monte Carlo method in the part of the scatter distribution corresponding to the locations of the sources while the DW technique gave lower estimates of the scatter distribution. Further comparisons of four scatter correction methods: DW, DPW, TEW and CV were also performed using simple phantoms and a clinically realistic source distribution simulating brain imaging. ${ }^{186}$ The authors concluded that performing scatter correction is essential for accurate quantification, and that all four methods yield a good, but not perfect, scatter correction. Buvat ${ }^{187}$ compared nine scatter correction methods based on spectral analysis. Simulations and physical phantom measurements were also used to compare the accuracy and noise properties of the transmission-dependent convolution subtraction and the TEW scatter correction techniques. ${ }^{188}$ The TEW had the worst signal-to-noise ratio in the heart chamber of a simulated chest phantom.

In the PET imaging world, Compton scattering effects in water on profiles of activity have been simulated. ${ }^{189}$ Figure 10(a) shows a comparison between measured and simulated single energy spectra of the ECAT-953B PET scanner. An energy resolution of $23 \%$ FWHM has been assumed, since this is the typical value for BGO block detectors. ${ }^{135}$ An energy pulse-height distribution obtained by simulation of a line source in the center of a water-filled cylindrical phantom where scattered events have been separated according to the number of scatterings is also shown [Fig. 10(b)]. The accuracy of experimental methodologies used for scatter fraction and scatter pair spatial distribution determination were evaluated using the Monte Carlo method. ${ }^{190}$ Figure 11 shows comparisons between measured and simulated scatter fractions as a function of the lower energy threshold. ${ }^{191}$ Barney ${ }^{192}$ developed an analytical simulation for single and multiplescattered gamma rays in PET. The multiple-scatter model showed good agreement with a Monte Carlo simulation of total object scatter. The authors also proposed a scatter correction method which uses the analytical simulation and exploits the inherent smoothness of the scatter distribution to account for three-dimensional effects in scatter distribution and object shapes. Scatter components in PET divided into primaries, object scatter, gantry scatter and mixed scatter and their effects on the degradation of reconstructed images were also investigated. ${ }^{193}$ Quantification of those components for a small animal PET prototype were also reported. ${ }^{194}$ A Monte Carlo study of the acceptance to scattered events in a depth encoding large aperture camera made of position encoding blocks modified to have DOI resolution through a variation in the photopeak pulse height was performed. ${ }^{191}$ It was reported that the poorer discrimination of object scatters with depth sensitive blocks does not lead to a dramatic increase of the scatter fraction.

Although several approaches have been proposed for scat- 


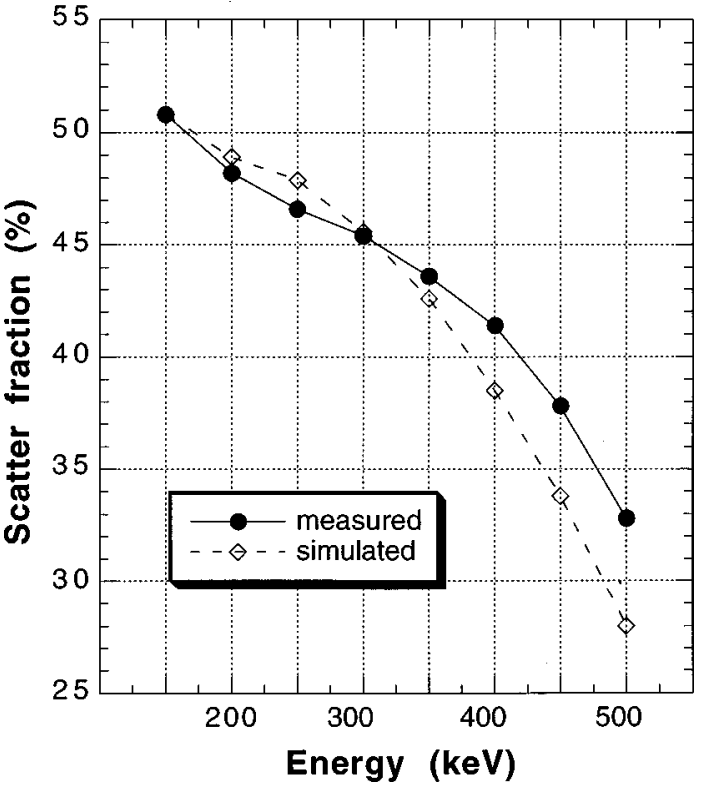

FIG. 11. A comparison between measured and simulated scatter fractions in the ECAT-953B as a function of the lower energy threshold (reprinted with permission from Ref. 191).

ter correction for 3D PET, six basic approaches have been taken to this correction: multi-energy window approaches, integral transformation approaches, an approach relying on an auxiliary, septa extended scan, curve-fitting approaches, model-based approaches, and direct Monte Carlo techniques. Levin $^{195}$ developed a correction method that uses the 3D reconstructed image volume as the source intensity distribution for a photon-tracking Monte Carlo simulation. The history of each annihilation photon's interactions in the scattering medium is followed, and the sinograms for the scattered and unscattered photon pairs are generated in a simulated $3 \mathrm{D}$ PET acquisition. The calculated scatter contribution is used to correct the original data set. Monte Carlo techniques were used to estimate "best possible" weighting functions for different energy-based scatter correction schemes and to examine the optimal number of energy windows for $\mathrm{NaI}(\mathrm{Tl})$ and BGO scintillators. ${ }^{196}$ Ollinger ${ }^{72}$ developed a model-based scatter correction method that uses a transmission scan, an emission scan, the physics of Compton scatter and a mathematical model of the scanner in a forward calculation of the number of events for which one photon has undergone a single Compton interaction. A single-scatter simulation technique for scatter correction where the mean scatter contribution to the net true coincidence data is estimated by simulating radiation transport through the object was also suggested and validated using human and chest phantom studies. ${ }^{73}$

\section{E. Dosimetry and treatment planning}

There is no doubt that the area where early Monte Carlo calculations in the field have been performed is dosimetry modeling and computations. ${ }^{10}$ The approach adopted by the Medical Internal Radiation Dose (MIRD) committee was first proposed in 1968 and published in a series of supple- ments to the Journal of Nuclear Medicine as different pamphlets. ${ }^{197-199}$ Some of these pamphlets made extensive use of Monte Carlo calculations to derive specific absorbed fractions for electron and photon sources uniformly distributed in organs of mathematical phantoms. Cristy ${ }^{200}$ demonstrated that the reciprocity theorem which states that for any pair of regions in a uniform isotropic or uniform scatterless model, the specific absorbed fraction is independent of which region is designated source and which is designated target may also be valid for heterogeneous phantoms for certain conditions. Comparisons between measured and calculated doses when the uncertainties associated with both techniques are considered validated the experimental validity of this approach. $^{201}$ Other approaches using the MIRD formalism have also been proposed. ${ }^{202}$ Poston $^{203}$ calculated photon specific absorbed fractions for both the Cristy and Eckerman gastrointestinal tract and their revised model and reported differences between electron absorbed fraction values with and without electron tracking. The calculation of absorbed fractions for positron emitters relevant to neurologic studies were also reported. ${ }^{204}$ Interest in Monte Carlo-based dose calculations with $\beta$-emitters has been revived with the application of labeled monoclonal antibodies to RIT.

In a review article on tumor dosimetry for $\beta$-emitters, Leichner and $\mathrm{Kwok}^{205}$ divided the various approaches into several classes, namely numerical, analytical or Monte Carlo. It is also necessary to consider hybrid approaches, namely numerical approaches using Monte Carlo data. The use of Monte Carlo codes enables the absorbed fraction of energy to be calculated directly for a given radionuclide relative to its geometry and emission spectrum. This can be done for relatively simple geometries ${ }^{206}$ but the main trend of Monte Carlo approaches is that they allow complex simulations involving inhomogeneities. ${ }^{48,207-211}$ Sometimes, the Monte Carlo technique is used just to simulate random distribution of sources or targets whereas the actual dosimetric calculation is performed using dose-point kernels. $^{212,213}$ Mono-energetic dose-point kernels which indicate variations in energy delivered at a distance from mono-energetic photon or electron point sources are commonly used data sets. The scaled point kernel $F\left(x / r_{0}, E_{0}\right)$ is defined by the equation

$$
F\left(\frac{x}{r_{0}}, E_{0}\right)=4 \Pi \rho x^{2} \Phi\left(x, E_{0}\right)
$$

where $\rho$ is the density of the medium, $r_{0}$ is the range in the continuous slowing down approximation (CDSA) at energy $E_{0}$ and $\Phi\left(x, E_{0}\right)$ is the specific absorbed fraction. These point kernels are calculated from Monte Carlo codes. Three Monte Carlo codes are often reported in the literature, ETRAN, ${ }^{197,198,214}$ ACCEPT $^{215}$ and EGS4. ${ }^{207,216,217}$ Although results obtained with ETRAN versions prior to 1986 may differ from those obtained with EGS4, ${ }^{40}$ due to an incorrect sampling of the energy-loss straggling in ETRAN, there are no important differences in the results obtained with these two codes. It must be noted that the results reported in MIRD pamphlet $7,{ }^{198}$ do not share the error described above. One 


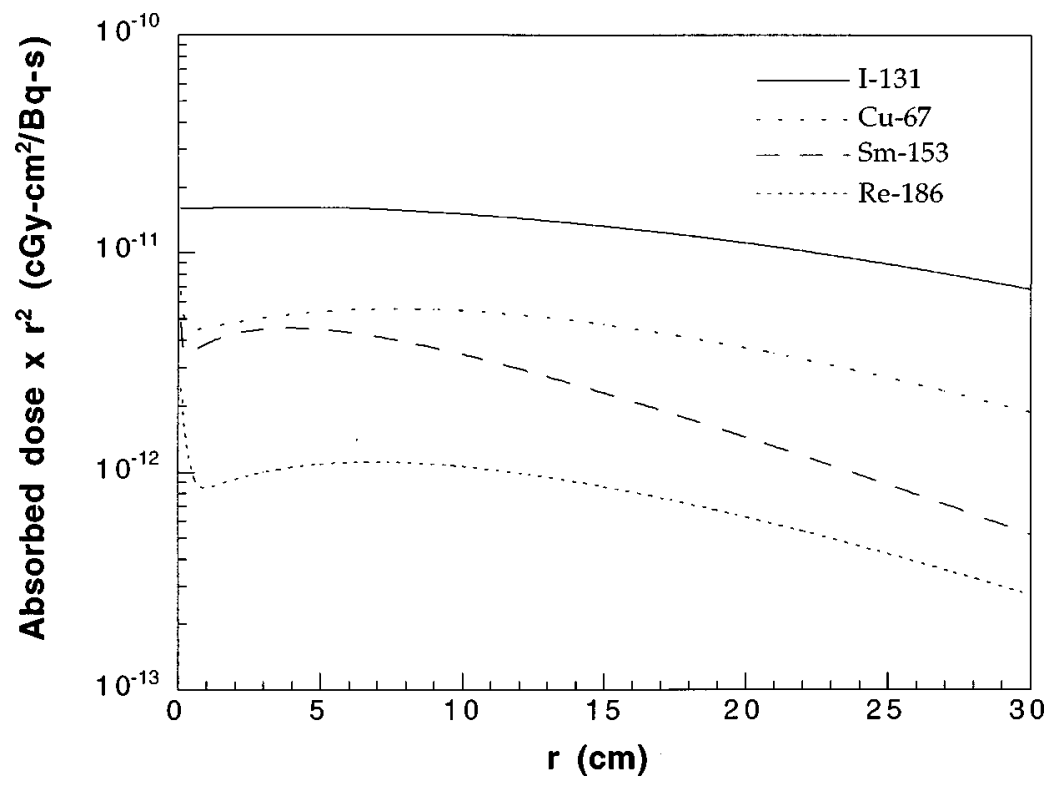

FIG. 12. Dose kernels generated in an infinite water medium for radionuclides of potential interest in radioimmunotherapy.

major limitation in applying these codes to dosimetry of $\beta$-emitting radionuclides is that they cannot deal satisfactorily with electron energies below $10 \mathrm{keV}{ }^{40}$ The EGS4 code was also used to characterize the spatial and energy distribution of bremsstrahlung radiation from beta point sources important to RIT in water. ${ }^{217}$ This study provided the initial data required for modeling and analyzing the scatter, attenuation, and image formation processes in quantitative imaging of bremsstrahlung for RIT dosimetry.

Leichner ${ }^{218}$ proposed a unified approach to photon and $\beta$-particle dosimetry. This approach is based on a fit of Berger's tables for photons ${ }^{197}$ and electrons. ${ }^{198}$ The empirical function proposed is equally valid for photons and $\beta$-particles. Therefore both point-kernel and Monte Carlo techniques can be effectively employed to calculate absorbed dose to tissue from radionuclides that emit photons or electrons. The latters are much computationally intensive, however, point-kernel methods are restricted to homogeneous tissue regions that can be mathematically described by analytical geometries, whereas Monte Carlo methods have the advantage of being able to accommodate heterogeneous tissue regions with complex geometric shapes. Recently, Furhang $^{219}$ generated photon point dose kernels and absorbed fractions in water for the full photon emission spectrum of radionuclides of interest in nuclear medicine, by simulating the transport of particles using Monte Carlo techniques. The kernels were then fitted to a mathematical expression. Figure 12 shows dose kernels generated in an infinite water medium for selected radionuclides of potential interest in RIT. ${ }^{219}$

The most recently available version of the MIRDOSE3 code, ${ }^{220}$ developed by the Radiation Internal Dose Information Center (Oak Ridge Institute for Science and Education) allows the calculation of the absorbed dose as well as the effective dose and the effective dose equivalent. The program deals with three phantoms representing the pregnant woman at 3,6 and 9 months of gestation and allows the dose delivered to the fetus to be calculated at different stages of growth. A phantom of the adult woman has also been included in the program which differs from that of the 15-yearold adolescent. The MABDOS program ${ }^{221}$ starting with a reference man allows for the definition of a spherical tumor target and "on the fly" Monte Carlo calculations to be made. ${ }^{222}$ This code was also used to show that neglecting the photon contribution from ${ }^{131} \mathrm{I}$ photon spectrum underestimates the tumor dose by $10-25 \% .{ }^{223}$

Akabani ${ }^{224}$ used EGS4 Monte Carlo calculations to estimate absorbed doses to the blood and to the surface of the blood vessel wall as well as to a mathematical model of a Haversian canal. ${ }^{225}$ Calculation of the dose to the upper spine region near the thyroid resulting from the administration of $3700 \mathrm{MBq}$ of ${ }^{131} \mathrm{I}$ and assuming a thyroid uptake of $10 \%$ was also performed. ${ }^{226}$ A Monte Carlo model has also been developed for the simulation of dose delivery to skeletal metastases by the bone surface-seeking radiopharmaceutical ${ }^{186} \operatorname{Re}(\mathrm{Sn})$ HEDP to optimize treatment planning and dose response evaluations of therapeutic bone-seeking radiopharmaceuticals. ${ }^{227}$ Beta-particle dosimetry of various radionuclides used in radiation synovectomy, an intraarticular radiation therapy to treat rheumatoid arthritis was also estimated using the EGS4 Monte Carlo code. ${ }^{228,229}$

It seems as if we are on the way to a more and more personalized human dosimetry with radiolabeled antibody dosimetry one of the aims. The dose distribution pattern is often calculated by generalizing a point source dose distribution, ${ }^{230,231}$ but a direct calculation by Monte Carlo techniques is also frequently reported because it allows media of inhomogeneous density to be considered. ${ }^{232}$ The development of a 3D treatment planner based on SPECT/PET imaging is an area of considerable research interest and several dose calculation algorithms have been developed. ${ }^{223}$ Figure 13 lists the essential steps required in developing a 3D treatment planning program for RIT. Projection data acquired from an emission tomographic imaging system are 


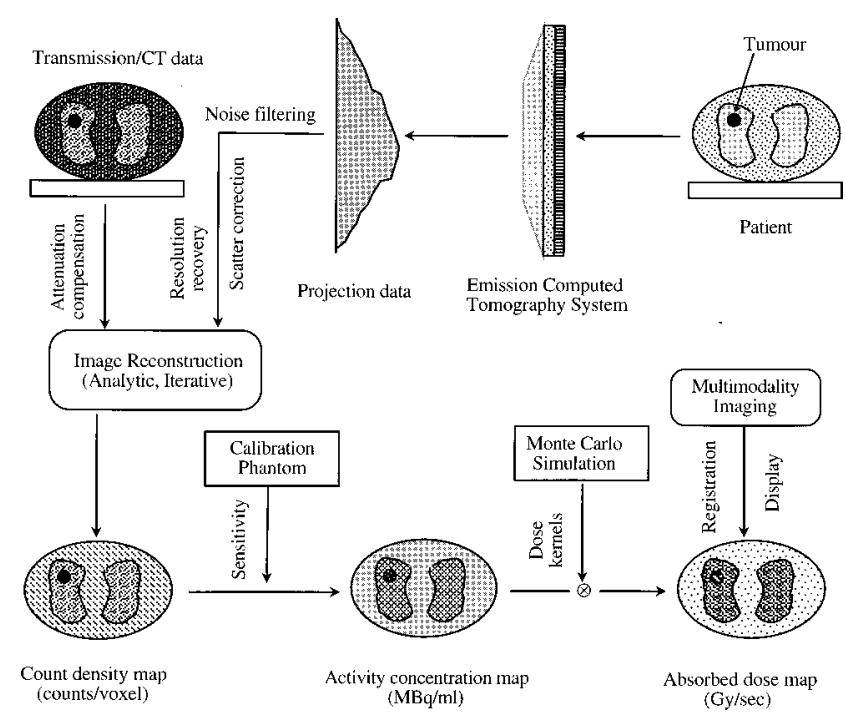

FIG. 13. A diagram showing the essential steps required in developing a three-dimensional internal dosimetry program based on quantitative emission computed tomography.

processed to reconstruct transverse section images which yields a count density map of source regions in the body. This count density is converted to an activity map using the sensitivity derived from a calibration phantom. In the final step, this activity distribution is converted to a dose rate or dose map by convolving the activity distribution with dosepoint kernels or by direct Monte Carlo calculations. To elaborate a treatment plan for an individual patient, prospective dose estimates can be made by using a tracer activity of radiolabeled antibody to obtain biodistribution information prior to administration of a larger therapeutic activity. The clinical implementability of treatment planning algorithms will depend to a significant extent on the time required to generate absorbed dose estimates for a particular patient.

In particular, Sgouros ${ }^{213}$ proposed real 3D treatment planning for RIT in which patient data (cumulative activity voxels) are convolved with dose-point kernels in order to determine the isodose distribution. ${ }^{233,234}$ This is then superimposed on the target visualized in 3D by computerized tomography (CT) or MRI. The methodology was extended later to develop a dosimetry algorithm based on a Monte Carlo procedure that simulates photon and electron transport and scores energy depositions within the patient. ${ }^{50,231,235}$

Microdosimetric approaches are required when the relative deviations from the mean of the local dose in the target exceed 20\%. Humm ${ }^{236}$ developed a full Monte Carlo simulation of the stochastic variation of particle hits and energy deposition in cell nuclei under two extreme geometric conditions, namely, when ${ }^{211} \mathrm{At}$ is retained in the capillary and when it is homogeneously distributed in the tumor. A method which allows dose calculations to be made to individual target cells in different regions of mouse bone marrow exposed to alpha particles emitted from bone was also developed. $^{237}$

\section{F. Pharmacokinetic modeling}

Pharmacokinetic modeling is a useful component for the estimation of cumulated activity in various source organs in the body. A few applications of Monte Carlo techniques have been reported in the field of pharmacokinetic modeling and are discussed in this section.

Casciari $^{238}$ developed a compartmental model of [F-18] fluoromisonidazole transport and metabolism to compute the volume average kappa in tissue regions from [F-18] fluoromisonidazole PET time-activity data and characterized it using Monte Carlo simulations and PET time-activity data. This model was able to accurately determine kappa for a variety of computer generated time-activity curves, including those for hypothetical heterogeneous tissue regions and poorly perfused tissue regions. Compartmental models allow also the in vivo analysis of radioligand binding to receptor sites in the human brain. Benzodiazepine receptor binding was studied using a three-compartmental model. ${ }^{239}$ The validity of the results of the coefficient of variation of each parameter were verified with statistical results provided by Monte Carlo simulation. Burger ${ }^{240}$ examined the possibility of mathematical metabolite correction, which might obviate the need for actual metabolite measurements. Mathematical metabolite correction was implemented by estimating the input curve together with kinetic tissue parameters. The general feasibility of the approach was evaluated in a Monte Carlo simulation using a two tissue compartment model. A simplified approach involving linear-regression straight-line parameter fitting of dynamic scan data was developed for both specific and nonspecific models. ${ }^{241}$ Monte-Carlo simulations were used to evaluate parameter standard deviations, due to data noise, and much smaller noise-induced biases. The authors reported good agreement between regression and traditional methods.

Welch $^{242}$ investigated and quantified the effect of typical SPECT system resolution and photon counting statistics on the bias and precision of dynamic cardiac SPECT parameters. The simulation of dynamic SPECT projection data was performed using a realistic human torso phantom assuming both perfect system resolution and a system resolution typical of a clinical SPECT system. The results showed that the rate constant characterizing the washing of activity into the myocardium is more sensitive to the region of interest position than is the washout rate constant, and that the main effect of increased photon noise in the projection data is to decrease the precision of the estimated parameters.

Computer simulations demonstrate that an estimation of the kinetic parameters directly from the projections is more accurate than the estimation from the reconstructed images. ${ }^{243}$ A strategy for the joint estimation of physiological parameters and myocardial boundaries was proposed and evaluated by simulated myocardial perfusion studies based on a simplified heart model. ${ }^{244} \mathrm{~A}$ method allowing the estimation of kinetic parameters directly from SPECT conebeam projections was also proposed and validated with a simulated chest phantom. ${ }^{245}$ The results showed that myocardial uptake and washout parameters estimated by conven- 
tional analysis of noiseless simulated cone-beam data had biases ranging between 3-26\% and $0-28 \%$, respectively, while uncertainties of parameter estimates with this method ranged between $0.2-9 \%$ for the uptake parameters and between $0.3-6 \%$ for the washout parameters.

\section{OBJECT MODEL AND SOFTWARE PHANTOMS}

Mathematical descriptions of human bodies and anthropomorphic phantoms are useful in radiation transport calculations. They are widely used in computer calculations of doses delivered to the entire body and to specific organs, and are valuable tools in the design and assessment of image reconstruction algorithms. Software phantoms modeled in imaging situations were historically limited to simple point, rod, and slab shapes of sources and attenuating media. Such simple geometries are useful in studying fundamental issues of scatter and attenuation, but clinically realistic distributions cannot be evaluated by such simple geometries. A precise modeling of the human body requires appropriate information on the location, shape, density and elemental composition of the organs or tissues.

\section{A. Object modeling}

Object modeling is fundamental for performing photon and electron transport efficiently by means of a Monte Carlo method. It consists of a description of the geometry and material characteristics for an object. ${ }^{246}$ The material characteristics of interest include density and energy-dependent crosssections. The modeling includes simple geometry (SG), shape-based (SB), and voxel-based (VB) approaches. The three approaches use a piecewise uniform distribution of object characteristics to model an object. With the SG model, an object is composed of a simple combination of primitives such as cylinders and spheres. The SB approach represents the boundaries of shapes by mathematical equations. Regular shapes such as sphere, cylinder, rectangular solid, etc. have been used to approximate irregularly-shaped regions. The VB approach discretizes an object into tiny cubes (voxels) with uniform characteristics. An object is thus represented by a union of voxels of the same size.

Extensions of SG and SB models such as the solid geometry-based (SGB) approach ${ }^{247}$ includes more primitives (ellipsoids, elliptic cylinders, tapered elliptic cylinders, rectangular solids, and their subsets: half, quarter, and eighth) and uses an inclusion tree data structure to provide relationships between primitives. These extensions provide simple irregular shape modeling. To allow anthropomorphic modeling the composite model ${ }^{248}$ which is an extension to the SGB approach adds to the primitives a voxelized rectangular solid primitive. An object model based on a combination of modified SG, SB and VB models without restriction in the combination set was also proposed. ${ }^{249}$ The data are structured in a hierarchical and adjacence tree associated with an efficient tree scanning to reduce the computation time. Combinatorial approaches to solid modeling, which describe complex structures as set-theoretic combinations of simple objects, are limited in their ease of use and place unrealistic constraints on

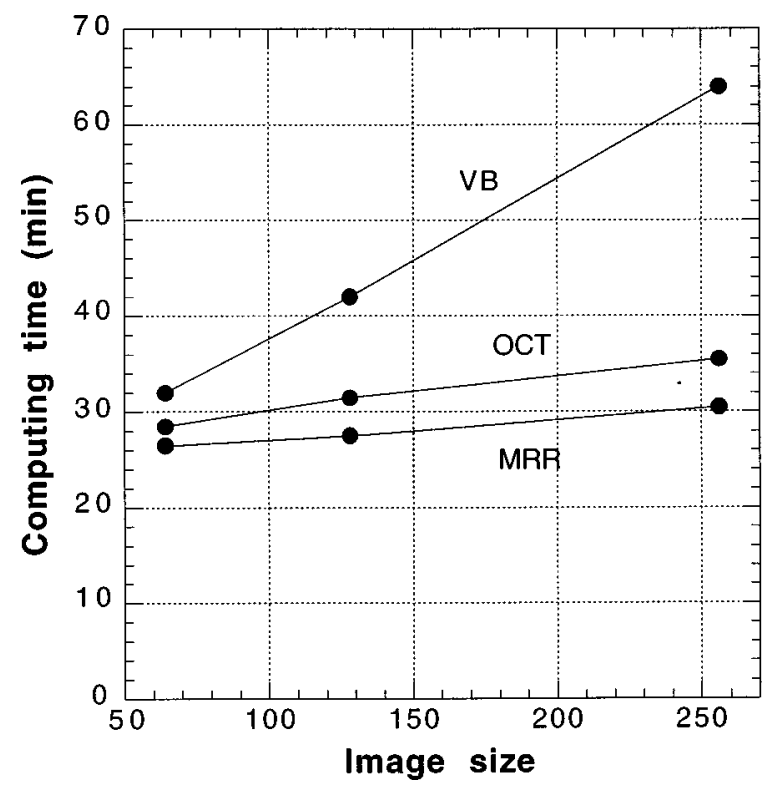

FIG. 14. Calculation times for VB, OCT, and MRR representation of an object (reprinted with permission from Ref. 246).

the geometric relations between objects such as excluding common boundaries. An approach to volume-based solid modeling has been developed which is based upon topologically consistent definitions of boundary, interior and exterior of a region. ${ }^{250}$ From these definitions, union, intersection and difference routines have been developed that allow involuted and deeply nested structures to be described as set-theoretic combinations of ellipsoids, elliptic cylinders, prisms, cones and planes that accommodate shared boundaries.

An octree-based method (OCT) which describes an object by using several sizes of cubic regions was proposed by Ogawa $^{251}$ to increase the calculation speed in photon transport since the number of voxels is much smaller than that of the VB approach. The "octree string" is generated from a set of serial cross-sections automatically. The same author developed a modeling method called the maximum rectangular region (MRR) method. ${ }^{246}$ In this approach, a MRR for a given voxel is selected within a homogeneous, irregularly shaped region from a set of cross-sections. The search is performed by checking the six sides of a box (MRR) including the voxel of interest. With the MRR representation of the object, high speed calculation of photon transport can be accomplished because an object can be described by means of fewer regions than in the VB or the OCT representation methods. Figure 14 illustrates the calculation time required for the VB OCT and MRR approaches for different image matrix sizes.

\section{B. Anthropommorphic phantoms}

Modeling of imaging and other medical applications is best done with phantom models that match the gross parameters of an individual patient. Computerized anthropomorphic phantoms can either be defined by mathematical (analytical) functions, or digital volume arrays. The mathematical specifications for phantoms that are available assume a spe- 
cific age, height and weight. People, however, exhibit a variety of shapes and sizes. In the first MIRD pamphlets, several organs including the skeletal system, were represented schematically using geometric forms (cylinders, cones and ellipsoids). ${ }^{199}$ The representation of internal organs with this mathematical phantom is very crude since the simple equations can only capture the most general description of the organ's position and geometry. ${ }^{202} \mathrm{~A}$ version of this phantom has been updated to include female organs. ${ }^{252}$ The most studied phantom is defined as the reference man weighing $70 \mathrm{~kg} .{ }^{253}$

Mathematical phantoms are still evolving and are being constantly improved. The heterogeneity of the body has been taken into account by including soft tissues, bone and lungs with different compositions and densities. For certain organs such as the stomach and the bladder, a distinction should be made between the organ contents and the organ wall. A revised head and brain models was developed by Bouchet. ${ }^{254}$ Unlike previous head models, the neck and head are treated as two separate compartments. The neck is represented by a circular cylinder. It is topped by a cylindrical head region cut in the back by a cone, so that its bottom base coincides with the top of the neck. Two vertical planes on the back join the cone to the cylinder of the head. The top of the head is defined by a half ellipsoid. The trunk region of the SnyderFisher phantom without its internal organs is incorporated into the model. Based on the atlas of sectional human anatomy, a 3D computer model of a human torso, including four cavities of the heart, two lobes of the lung and the body surface and a 3D model of the myocardium was developed. ${ }^{255}$ The torso model, with more than 10000 surface triangles, depicts the structures and appropriate proportions of the internal organs, especially of the heart.

The Mathematical CArdiac Torso (MCAT) phantom is an anthropomorphic phantom, developed at the University of North Carolina at Chapel Hill, that has been used in emission computed tomography imaging research. ${ }^{256}$ Using mathematical formulas, the size, shape and configurations of the major thoracic structures and organs such as the heart, liver, breasts and rib cage are realistically modeled for imaging purposes. Though anatomically less realistic than phantoms derived from CT or MR images of patients, the MCAT phantom has the advantage that it can be easily modified to simulate a wide variety of patient anatomies. In addition, the MCAT phantom simulates a dynamic, beating heart including changes in myocardial wall thickness, changes in chamber volumes, apical movement and heart rotation during the cardiac cycle. The phantom consists of two physical models: a 3D distribution of attenuation coefficients and a 3D distribution of radionuclide uptake for the various thoracic organs. The 3D attenuation coefficient phantom classifies all thoracic tissues into one of 5 types: muscle (vasculature and other soft tissues), lung, fat (such as in the breasts), trabecular bone and cortical bone. The MCAT phantom has become a valuable tool in imaging studies where reasonably realistic, but anatomically variable patient data needs to be simulated. The graphic in Fig. 15 illustrates the MCAT phantom. The
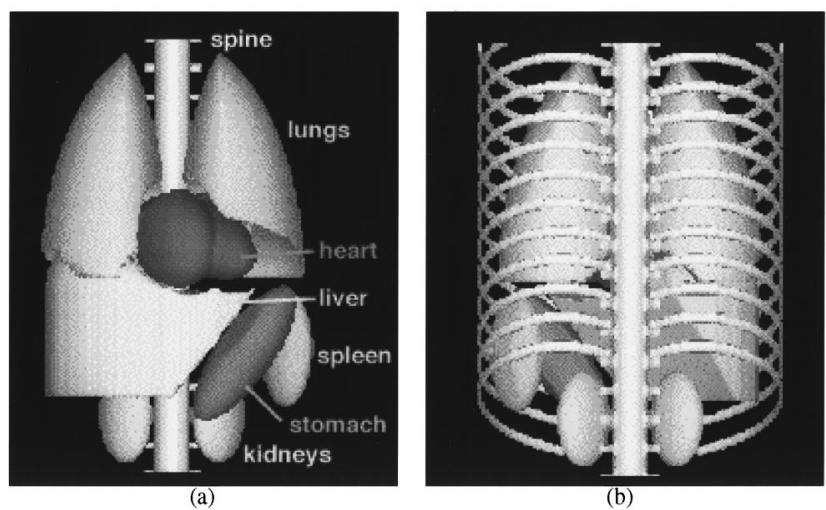

FIG. 15. Surface rendered images of the 3D MCAT phantom developed at Chapel Hill. (a) Anterior view with outer body surface and ribs removed to show the various organs modeled. (b) Posterior view with the rib cage present (reprinted with permission from Ref. 256).

graphic is a volume-rendering of anterior and posterior views with some sections removed for visualization purposes.

Some calculations make use of more accurate representations of individuals based on volumetric scans, such as CT, MRI, and PET. As an improvement to the mathematical anthropomorphic phantoms, a new family of phantoms was constructed from CT data. ${ }^{257}$ The human phantoms present advantages towards the location and shape of the organs, in particular, the hard bone and bone marrow. A physical brain phantom has also been developed to simulate the activity distributions found in the human brain in the cerebral blood flow and metabolism studies currently employed in PET. ${ }^{258}$ The phantom utilizes thin layers of Lucite to provide apparent relative concentrations of 4,1 and 0 for gray matter, white matter and ventricles, respectively, in the brain. A clinically realistic source distribution simulating brain imaging was created in digital format. ${ }^{259} \mathrm{Zubal}^{59,260}$ developed a typical anthropommorphic VB adult phantom by manual segmentation of CT transverse slices of a living human male performed by medical experts. A computerized 3D volume array modeling all major internal structures of the body was then created. Each voxel of the volume contains an index number designating it as belonging to a given organ or internal structure. These indexes can then be used to assign a value, corresponding to, e.g., density or activity. Two versions of the phantom exist, representing either the complete human torso with an isotropic voxel resolution of $1.5 \mathrm{~mm}$, or a dedicated head phantom with $0.5 \mathrm{~mm}$ voxel size. The dedicated brain phantom was created from the high resolution MRI scans of a human volunteer. This volume array represents a high resolution model of the human anatomy and serves as a VB anthropomorphic phantom.

\section{MONTE CARLO COMPUTER CODES}

Many Monte Carlo programs have been in use in the field of nuclear imaging ${ }^{14,16,17,261}$ and internal dosimetry, ${ }^{50,220,232,234}$ with many of them available in the 
TABLE II. Key features of Monte Carlo codes used in nuclear medical imaging.

\begin{tabular}{|c|c|}
\hline MC code & General description \\
\hline EGS4 (Ref. 262) & $\begin{array}{l}\text { Coupled photons/electrons transport in any material } \\
\text { through user specified geometries. Simulation of } \\
\text { imaging systems not specifically included and requires } \\
\text { an extensive amount of user programming in MORTRAN. }\end{array}$ \\
\hline $\begin{array}{l}\text { ITS including TIGER, CYLTRAN, } \\
\text { and ACCEPT (Ref. 263) }\end{array}$ & $\begin{array}{l}\text { Coupled photons/electrons transport in any material } \\
\text { through slabs, cylinders or combinatorial. Simulation of } \\
\text { imaging systems not specifically included and requires } \\
\text { an extensive amount of user programming in FORTRAN. }\end{array}$ \\
\hline MCNP (Ref. 264) & $\begin{array}{l}\text { Coupled neutrons/photons/electrons transport in any } \\
\text { material through user generalized geometry. Simulation } \\
\text { of imaging systems not specifically included and } \\
\text { requires an extensive amount of user programming in } \\
\text { FORTRAN. }\end{array}$ \\
\hline GEANT (Ref. 43) & $\begin{array}{l}\text { Coupled photons/electrons transport in any material } \\
\text { through combinatorial geometry. Simulation of imaging } \\
\text { systems not specifically included and requires an } \\
\text { extensive amount of user programming in FORTRAN. }\end{array}$ \\
\hline SIMSET (Ref. 21) & $\begin{array}{l}\text { Photons transport in any material through voxel-based } \\
\text { phantoms. Simulation of SPECT and PET imaging } \\
\text { systems included. User modules written in C could be } \\
\text { linked. }\end{array}$ \\
\hline SIMIND (Ref. 15) & $\begin{array}{l}\text { Photons transport in any material through voxel-based } \\
\text { phantoms. Simulation of SPECT imaging systems } \\
\text { included. User modules written in FORTRAN could be } \\
\text { linked. }\end{array}$ \\
\hline SIMSPECT (Ref. 265) & $\begin{array}{l}\text { Coupled photons/electrons transport in any material } \\
\text { through voxel-based phantoms. Simulation of SPECT } \\
\text { imaging systems included. User modules written in } \\
\text { FORTRAN/C could be linked. }\end{array}$ \\
\hline MCMATV (Ref. 266) & $\begin{array}{l}\text { Photons transport in any material through voxel-based } \\
\text { phantoms. Simulation of SPECT imaging systems } \\
\text { included. User modules written in FORTRAN could be } \\
\text { linked. }\end{array}$ \\
\hline PETSIM (Ref. 20) & $\begin{array}{l}\text { Photons transport in any material through shape-based } \\
\text { phantoms. Simulation of PET imaging systems included. } \\
\text { User modules written in FORTRAN could be linked. }\end{array}$ \\
\hline EIDOLON (Ref. 135) & $\begin{array}{l}\text { Photons transport in any material through shape-based or } \\
\text { voxel-based phantoms. Simulation of 3D PET imaging } \\
\text { systems included. User modules written in C/Objective- } \\
\mathrm{C} \text { could be linked. }\end{array}$ \\
\hline
\end{tabular}

public domain., ${ }^{9,10}$ Table II (Refs. 262-266) lists Monte Carlo codes widely used together with a short description of their key features.

EGS4. The electron gamma shower (EGS) computer code system is a general purpose package for Monte Carlo simulation of the coupled transport of electrons and photons in an arbitrary geometry for particles with energies from a few $\mathrm{keV}$ up to several $\mathrm{TeV} .{ }^{262}$ The code represents the state-ofthe-art of radiation transport simulation because it is very flexible, well-documented and extensively tested. Some have referred to the EGS code as the de facto gold standard for clinical radiation dosimetry EGS is written in MORTRAN, a FORTRAN pre-processor with powerful macro capabilities. EGS is a "class II'" code that treats knock-on electrons and bremsstrahlung photons individually. Such events require predefined energy thresholds and pre-calculated data for each threshold, determined with the cross-section generator PEGS.

ITS. The Integrated TIGER Series (ITS) of coupled electron/ photon Monte Carlo transport codes is a powerful tool for determining state-of-the-art descriptions of the production and transport of the electron/photon cascade in timeindependent, multi-material, multi-dimensional environments. ${ }^{263}$ ITS is a collection of programs sharing a common source code library that can solve sophisticated radiation transport problems. A total of eight codes are in the collection which can be split into six groups: the TIGER codes (for 1D slab geometries), the CYLTRAN codes (for 2D cylindrical geometries), the ACCEPT codes (for arbitrary 3D geometries), the standard codes (for normal applications), the $\mathrm{P}$ codes (for applications where enhanced ionization/relaxation procedures are needed), and the $\mathrm{M}$ codes (for applications which involve 2- or 3D macroscopic electromagnetic fields). The user selects the appropriate code from the library and supplies it with any special requirements and the physical description of the problem to be solved in an input file.

MCNP. MCNP is a general-purpose Monte Carlo code that can be used for neutron, photon, electron or coupled neutron/ 
photon/electron transport. ${ }^{264}$ The code treats an arbitrary three-dimensional configuration of materials in geometric cells bounded by first- and second-degree surfaces and fourth-degree elliptical tori. For photons, the code takes account of incoherent and coherent scattering, the possibility of fluorescent emission after photoelectric absorption, absorption in pair production with local emission of annihilation radiation and bremsstrahlung. A continuous slowing down model is used for electron transport that includes positrons, $\mathrm{k}$-shell x-rays, and bremsstrahlung but does not include external or self-induced fields. Important features that make MCNP very versatile and easy to use include a powerful general source, criticality source, and surface source; both geometry and output tally plotters; a rich collection of variance reduction techniques; a flexible tally structure; and an extensive collection of cross-section data.

GEANT. The GEANT package was originally designed for high energy physics experiments, but has found applications also outside this domain in the areas of medical and biological sciences, radiation protection and astronautics. ${ }^{43}$ The main applications of GEANT are the transport of particles through an experimental setup for the simulation of detector response and the graphical representation of the setup and of the particle trajectories. The two functions are combined in the interactive version of GEANT. This is very useful, since the direct observation of what happens to a particle inside the detector makes the debugging easier and may reveal possible weakness of the setup.

SIMSET. The simulation system for emission tomography (SIMSET) is a software application designed to perform Monte Carlo simulation of photon creation and transport through heterogeneous attenuators for both SPECT and PET. ${ }^{21}$ The package has been in the public domain since the beginning of 1996 and includes the photon history generator (PHG), the object editor, a collimator module and detection and binning modules. The PHG is the module that generates and tracks photons within the FOV of the tomograph being simulated. The code is continuously being improved including, for instance, the implementation of incoherent scattering, random events detection and simulation of coincidence imaging using conventional dual-head gamma cameras.

SIMIND. The SIMIND code simulates a clinical SPECT scintillation camera and can easily be modified for almost any type of calculation or measurement encountered in SPECT imaging, ${ }^{15}$ including transmission imaging. ${ }^{168}$ The entire code has been written in FORTRAN-90 and includes versions that are fully operational on VAX-VMS, most UNIX platforms and on MS-DOS (Lahey LF90 compiler). In summary, the code works as follows: photons emitted from simulated decay in the phantom are followed step by step towards the scintillation camera. SIMIND includes an accurate treatment of photon interaction in the phantom, a protecting layer and in the crystal of the detector. The simulation of back-scattering from light guides and photomultipliers is also included. Different types of collimators can be selected. SIMIND can take advantage of anthropomorphic voxel-based phantoms developed for simulating realistic imaging situations. The program has been shared among several groups and has been found a very useful research tool. The SIMIND code has been widely used for collimators design ${ }^{111}$ and to evaluate attenuation and scatter correction techniques. ${ }^{174,176-179,185-186}$

SIMSPECT. The SIMSPECT code developed at MIT is based on the MCNP Monte Carlo transport code for photon tracking and interaction algorithms. It has been extensively modified to allow complete collimator and source modeling and direct manipulation of the geometric and physical parameters encountered in SPECT imaging. ${ }^{18,265}$ The simulation package allows full tomographic simulation of data from physically realistic nonuniform and asymmetric 3D source objects. Photon transport in the detector crystal, light pipe and PMT's is not simulated. The use of positron emitters in SPECT imaging has also been modeled via SIMSPECT in order to better understand the potential improvements of different collimators design on $511 \mathrm{keV}$ gamma camera imaging.

MCMATV. The Monte Carlo Matrix Vectorized (MCMATV) program models photon transport in both homogeneous ${ }^{266}$ and heterogeneous media. ${ }^{267}$ The code is designed to model both projection data for simulated SPECT studies and to compute photon detection kernels, which can be used to build system matrices for use in matrix-based image reconstruction. ${ }^{139}$ The vectorized code is written in FORTRAN77 and run on a Stellar GS1000 computer for pipelined computations. It uses an event-based algorithm in which photon history data are stored in arrays and photon history computations are performed within DO loops. The code is adapted from a history-based Monte Carlo code in which photon history data are stored in scalar variables and photon histories computed sequentially. Without the use of the vector processor the event-based code is faster than the historybased code because of numerical optimization performed during conversion to the event-based algorithm.

PETSIM. A series of programs called PETSIM have been developed to model the source distribution and its attenuation characteristics, as well as the collimator and detectors in PET. ${ }^{20,44}$ The different modules are connected by compact gamma history files which are stored on a disk or tape. The storage of intermediate results on tape reduces simulation time, since most common source geometries need be generated only once. The simulation results include spectrum analysis, sensitivity to true coincident events, scattered coincident and single events and the effects of these parameters of detector dead-time. The sensitivities in multi-slice systems are presented as matrices of coincident crystal planes. The matrix shows the true count sensitivity and the scatter fraction together for each valid combination of planes. This presentation is very useful for assessing the effects of various degrees of inter-plane collimation. The spatial resolution analysis includes the effects of positron range, noncolinearity of the gamma rays, multiple interaction within the detectors, and the effects of quantization into single crystals in multiple-crystal block detectors. Each of these effects can be turned on or off without repeating the simulation. Single crystals, blocks and crystals with DOI encoding can be specified, so that the detector geometry can be optimized.

EIDOLON. The Monte Carlo simulator, EIDOLON, was de- 
veloped using modern software engineering techniques mainly for fully 3D PET imaging. ${ }^{22,135}$ The code was written in Objective-C, an object-oriented programming language based on ANSI C. The first version of the program was developed using the NextStep development environment. A modular design featuring dynamically loadable program elements or bundles was adopted for software design. The basic building block is a model element object class which allows us elements to be browsed, inspected, adjusted, created and destroyed through a graphical inspector. The user interface allows the user to select scanner parameters such as the number of detector rings, detector material and sizes, energy discrimination thresholds and detector energy resolution. It also allows us to choose either a complex anthropomorphic phantom or a set of simple 3D shapes, such as parallelepiped, ellipsoid or cylindroid for both the annihilation sources and the scattering media, as well as their respective activity concentrations and chemical compositions. The user has the possibility to view the reference source image and sinogram data sets as they are generated and are periodically updated. An implementation of the software on a high-performance parallel platform was also reported. ${ }^{38}$

\section{SCALAR VERSUS VECTORIZED AND PARALLEL MONTE CARLO SIMULATIONS}

Although variance reduction techniques have been developed to reduce computation time, the main drawback of the Monte Carlo method is that it is extremely time-consuming. To obtain the high statistics ( $\sim 10^{7}$ counts) required for image reconstruction studies requires us to track hundreds of millions of particles. Consequently, a large amount of CPU time (weeks or even months) may be required to obtain useful simulated data sets. The development of advanced computers with special capabilities for vectorized or parallel calculations opened a new way for Monte Carlo researchers. Parallel computers are becoming increasingly accessible to medical physicists. ${ }^{268}$ This allows research into problems that may otherwise be computationally prohibitive to be performed in a fraction of the real time that would be taken by a serial machine. Historically, however, most programs and software libraries have been developed to run on serial, single-processor computers. A modification or adaptation of the code is therefore a prerequisite to run it on a parallel computer. However, it is worth pointing out that among all simulation techniques of physical processes, the Monte Carlo method is probably the most suitable one for parallel computing since the results of photon histories are completely independent from each other. Moreover, computer aided parallelization tools designed to automate as much as possible the process of parallelizing scalar codes are becoming available. ${ }^{269}$ Although parallel processing seems to be the ideal solution for Monte Carlo simulation, very few investigations have been reported and only a few papers have been published on the subject. ${ }^{270}$

The theoretical peak performance of a computer is determined by counting the number of floating-point additions and multiplications that can be completed during period of time, usually the cycle time of the machine. ${ }^{271}$ Today's large machines measure their speed in GFlops $\left(10^{9}\right.$ operations/s). Each CPU generally contains 2 multiply and 2 add pipes. When all of these can be employed simultaneously as for instance, in a dot product or a vector update operation, 4 Flops/cycle can be attained. The Cray T90 has a cycle time of $2.2 \mathrm{~ns}$ and a maximum number of 32 processors, thus the peak performance is 4 operations $/ 1$ cycle $\times 1$ cycle $/ 2.2 \mathrm{~ns}$ $\times 32$ processors $=58.2$ GFlops. Easily portable Monte Carlo user codes are generally used for timing benchmark purposes on different computers. ${ }^{272}$ According to van der Steen and Dongarra, ${ }^{273}$ the classification of high-performance computers is based on the way instructions and data streams are arranged and comprises four main architectural classes. These include the following.

Single Instruction Single Data stream (SISD) machines. These are the conventional systems that contain one CPU and hence can accommodate one instruction stream that is executed serially. Nowadays many large mainframes may have more than one CPU but each of these execute instruction streams that are unrelated. Therefore, such systems still should be regarded as (multiple) SISD machines acting on different data spaces. Examples of SISD machines are for instance most workstations like those of DEC HewlettPackard and Sun Microsystems.

Single Instruction Multiple Data stream (SIMD) machines. Such systems often have a large number of processing units, ranging from 1,024 to 16,384 that all may execute the same instruction on different data in lock-step. So, a single instruction manipulates many data items in parallel. Examples of SIMD machines in this class are the CPP DAP Gamma II and the Alenia Quadrics. Another subclass of the SIMD systems are the vector processors which act on arrays of similar data rather than on single data items using specially structured CPUs. When data can be manipulated by these vector units, results can be delivered with a rate of one, two and in special cases of three per clock cycle. So, vector processors execute on their data in an almost parallel way but only when executing in vector mode. In this case they are several times faster than when executing in conventional scalar mode. For practical purposes, vector processors are therefore mostly regarded as SIMD machines. An example of such systems is, for instance, the Hitachi S3600.

Multiple Instructions Single Data stream (MISD) machines. Theoretically in these types of machines multiple instructions should act on a single stream of data. As yet, no practical machine in this class has been constructed nor are such systems easy to conceive.

Multiple Instructions Multiple Data streams (MIMD) machines. These machines execute several instruction streams in parallel on different data. The difference with the multi-processor SISD machines mentioned above lies in the fact that the instructions and data are related because they represent different parts of the same task to be executed. So, MIMD systems may run many sub-tasks in parallel in order to shorten the time-to-solution for the main task to be executed. There is a large variety of MIMD systems including those that behave very differently like a four-processor Cray 
Y-MP T94 and a thousand processor nCUBE 2S. An important distinction between two subclasses of systems should, however, be made: Shared memory (SM) and distributed memory (DM) systems.

(i) Shared memory systems. SM systems have multiple CPUs, all of which share the same address space. This means that the knowledge of where data is stored is of no concern to the user as there is only one memory accessed by all CPUs on an equal basis. Shared memory systems can be both SIMD or MIMD. Single-CPU vector processors can be regarded as an example of the former, while the multi-CPU models of these machines are examples of the latter. The Cray J90 and T90 series belong to this class of computers.

(ii) Distributed memory systems. In this case, each CPU has its own associated memory. The CPUs are connected by some network and may exchange data between their respective memories when required. In contrast to SM machines the user must be aware of the location of the data in the local memories and will have to move or distribute these data explicitly when needed. Again, DM systems may be either SIMD or MIMD. The first class of SIMD systems, mentioned above, operate in lock step and all have distributed memories associated to the processors. For the DM-MIMD systems again a subdivision is possible: those in which the processors are connected in a fixed topology and those in which the topology is flexible and may vary from task to task. The class of DM-MIMD machines is undoubtedly the fastest growing part in the family of high-performance computers.

Another trend that has come up in the last few years is distributed processing. This takes the DM-MIMD concept one step further: instead of many integrated processors in one or several boxes, workstations, mainframes, etc., are connected by Ethernet, for instance and set to work concurrently on tasks in the same program. Conceptually, this is not different from DM-MIMD computing, but the communication between processors is often orders of magnitude slower. Many commercial, and noncommercial packages to realize distributed computing are available. Examples of these are Parallel Virtual Machine (PVM), ${ }^{274}$ and Message Passing Interface (MPI). ${ }^{275}$ PVM and MPI have been adopted for instance by HP/Convex, SGI/Cray, IBM and Intel for the transition stage between distributed computing and Massively Parallel Processing (MPP) systems on the clusters of their favorite processors and they are available on a large amount of DM-MIMD systems and even on SM-MIMD systems for compatibility reasons. In addition, there is a tendency to cluster SM systems, for instance by HIPPI channels, to obtain systems with a very high computational power, e.g., the NEC SX-4 and the Convex Exemplar SPP-2000X have this structure, although the latter system could be seen as a more integrated example (the software environment is much more complete and allows SM addressing). Other interesting research systems like the Intel ASCI Option Red system at Sandia National Laboratory (with a measured performance of 1.3 TFlops), the CP-PACS at the University of Tsukuba (measured performance of 368 GFlops) and the Numerical Wind Tunnel at the National Aerospace Lab. in Japan (230
GFlops), are not marketed and only available at the Institutes mentioned and, therefore, not of much benefit to the supercomputer community at large. It is worth noting that the market of parallel and vector machines is highly evasive; the rate with which systems are introduced and disappear again is very high and therefore the information provided will probably be only approximately valid.

Sequential programs make the most effective use of the available processing power: they alone guarantee maximum use of the CPU. In parallel programs, communication management introduces an unavoidable overhead, resulting in less efficient use of the overall CPU power. Moreover, according to Amdahl's law, ${ }^{276}$ parallelization efficiency is decreased by a factor representing the fraction of operations that must be executed in sequential order. When this fraction reaches one we are confronted with a wholly unparallelizable code, and the speed-up is zero no matter how many processors are used. The efficiency of parallel programs is furthermore reduced by a factor equal to the fraction of processor idle time, which is highly dependent on the software parallelization techniques used by the programmer. Scalar or serial Monte Carlo codes track the history of one particle at a time, and the total calculation time is the sum of the time consumed in each particle history. Many Monte Carlo applications have characteristics that make them easy to map onto computers having multiple processors. Some of these parallel implementations require little or no inter-processor communication and are typically easy to code on a parallel computer. Others require frequent communication and synchronization among processors and in general are more difficult to write and debug. A common way to parallelize Monte Carlo is to put identical "clones"' on the various processors; only the random numbers are different. It is therefore important for the sequences on the different processors to be uncorrelated so each processor does not end up simulating the same data. ${ }^{277}$ That is, given an initial segment of the sequence on one process, and the random number sequences on other processes, we should not be able to predict the next element of the sequence on the first process. For example, it should not happen that if we obtain random numbers of large magnitude on one process, then we are more likely to obtain large numbers on another. In developing any parallel Monte Carlo code, it is important to be able to reproduce runs exactly in order to trace program execution.

Since a Monte Carlo particle history is a Markov chain, the next interaction or movement of a particle is always determined by the current state of the particle. The histories of two particles became identical only when the same random number sequence is used to sample the next state. To ensure that the seed tables on each processor are random and uncorrelated, Mascagni ${ }^{36}$ described a canonical form for initializing separate cycles of the Fibonacci generators. There are, however, many approaches to vectorized and parallel random number generation in the literature..$^{278-280}$ We can distinguish three general approaches to the generation of random numbers on parallel computers: centralized, replicated and distributed. In the centralized approach, a sequential generator is encapsulated in a task from which other tasks 

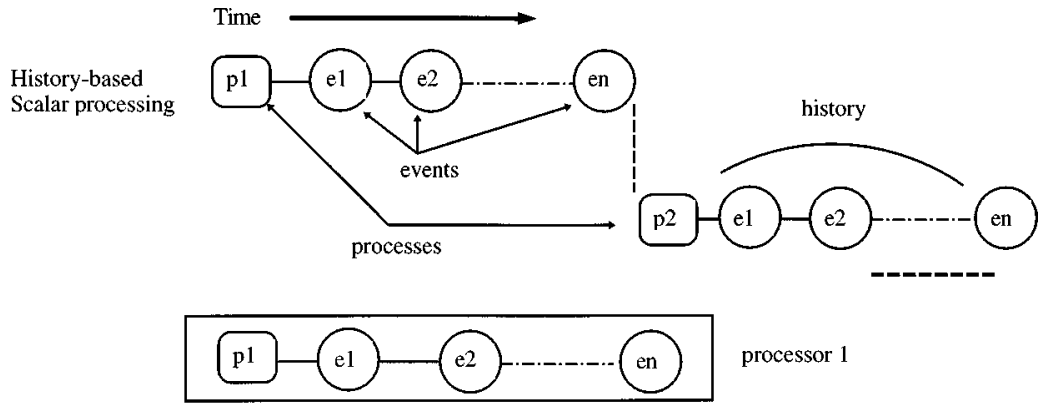
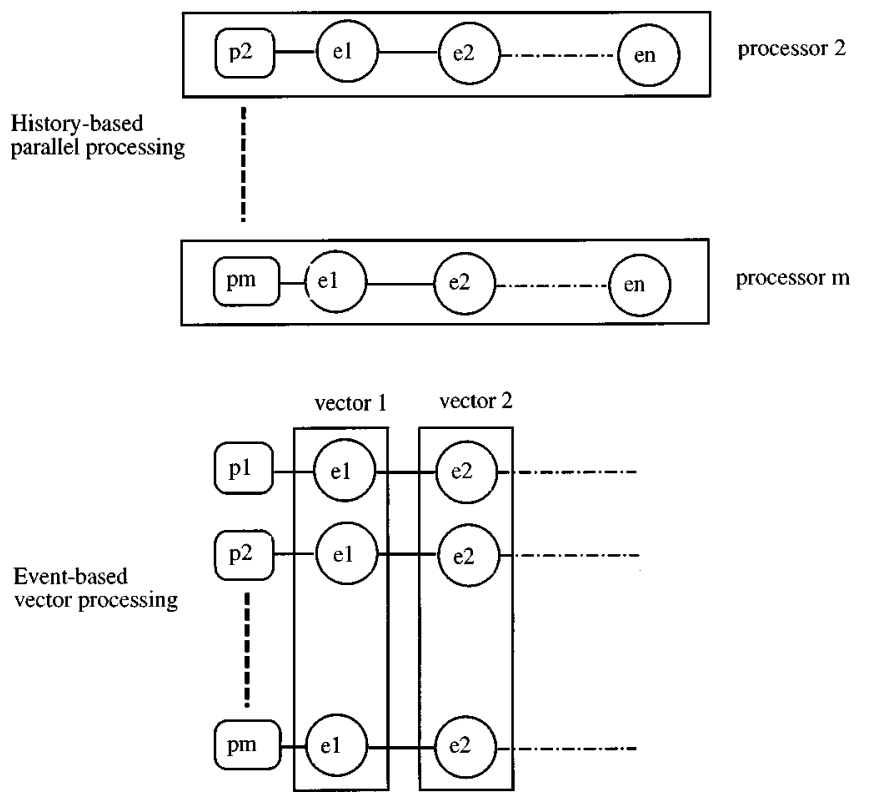

request random numbers. This avoids the problem of generating multiple independent random sequences, but is unlikely to provide a good performance. Furthermore, it makes reproducibility hard to achieve: the response to a request depends on when it arrives at the generator, and hence the result computed by a program can vary from one run to the next. In the replicated approach, multiple instances of the same generator are created (for example, one per task). Each generator uses either the same seed or a unique seed, derived, for example, from a task identifier. Clearly, sequences generated in this fashion are not guaranteed to be independent and, indeed, can suffer from serious correlation problems. However, the approach has the advantages of efficiency and ease of implementation and should be used when appropriate. In the distributed approach, responsibility for generating a single sequence is partitioned among many generators, which can then be parceled out to different tasks. The generators are all derived from a single generator; hence, the analysis of the statistical properties of the distributed generator is simplified. There are two possibilities for parallelisation of Monte Carlo codes.

(i) Particle or history-based parallelization: each particle is assigned to one of the parallel processes and the histories of assigned particles are processed within the process.

(ii) Task- or event-based parallelization: a process treats
FIG. 16. A comparison between history-based scalar processing, event-based vector processing and historybased parallel processing. In history-based scalar processing, one particle history is tracked at a time. In history-based parallel processing, each particle $\left(p_{1}, p_{2}, \ldots, p_{m}\right)$ is assigned to one process which tracks its complete history $\left(e_{1}, e_{2}, \ldots, e_{n}\right)$. In event-based vector processing, a process treats only part of each particle history $\left(e_{1}, e_{2}, \ldots, e_{n}\right)$ and particles $\left(p_{1}, p_{2}, \ldots, p_{m}\right)$ "flow" from process to process. only part of random walk events or tasks, thus particles "flow" from process to process according to their events.

The difference between the different algorithms is illustrated in Fig. 16. In a review of vectorized Monte Carlo, Martin and Brown ${ }^{281}$ described variations of event-based algorithms together with speed-up results published by different groups.

During the last two decades, investigations were carried out to run different Monte Carlo codes on multipletransputer systems, ${ }^{282,283}$ vector parallel supercomputers, ${ }^{266,267,284,285}$ parallel computers ${ }^{38,286,287}$ and a cluster of workstations in a local area network using PVM. ${ }^{288}$ There are large discrepancies in the performance ratio reported by different authors. In particular, Miura ${ }^{285}$ reported a speed-up of about 8 with the vectorized ESG4 code (EGS4V). A factor varying between 2.9 and 5.1 was also reported for MCMATV $^{266}$ depending on whether the detection of scattered photons is modeled or not. A linear decrease in computing time with the number of processors used was also reported with EIDOLON. ${ }^{38}$

\section{CONCLUSIONS AND FUTURE PROSPECTS}

Nuclear medicine has historically been the field in which most of the early Monte Carlo calculations in medical physics were performed. The large number of applications re- 
ferred to in this paper shows the usefulness and considerable success of the Monte Carlo method as a tool in different areas of nuclear imaging. The availability of Monte Carlo codes in the "public domain," developed and tested worldwide in a variety of applications by many users should provide confidence for their use as research tools in the different fields of nuclear imaging. The use of Monte Carlo calculations to validate scatter and attenuation correction methods has been useful. Furthermore, scatter images can, in principle, be calculated for any arbitrary source distribution. Simulated realistic emission images (including both primary and scatter) can be corrected for attenuation and scatter, and be compared with scatter- and attenuation-free ideal images. The accuracy in correction methods can thus be evaluated in an unbiased way since systematic errors can be controlled.

The lack of inherent error estimates and relatively slow convergence is a well known limitation of the Monte Carlo technique. In many situations, one can predict the statistical error (variance) on the estimated parameters from Monte Carlo simulations, and hence estimate the number of trials needed to achieve a given error. A few investigations have been carried out to quantify the accuracy of Monte Carlo simulations. However, some authors reported discrepancies between experimental measurements and results obtained with Monte Carlo programs. For example, it has been noticed that the measured energy spectra for a small point source has a larger magnitude low-energy tail than is obtained with Monte Carlo simulations. ${ }^{164,165}$ There are several possible explanations for this effect. The background radiation always present in scanning rooms is a possibility to be considered. A potential source of those events might be "scatter" from photomultiplier tubes back into the detection crystal. ${ }^{165}$ This effect could also be caused by the characteristics of the scinitillation camera electronics. ${ }^{164}$ Discrepancies also observed in quantification of parameters like the scatter fraction could be explained by the sharp energy threshold model used in most codes, ${ }^{70,191}$ i.e., the experimentally observed variations in crystal energy response is hardly taken into account in the analysis of simulated data. ${ }^{135}$ Therefore, it is important that most simulations of complex phantom geometries be matched with scatter-free simulations. There are other problems associated with the detection system which are too camera specific for Monte Carlo to be worthwhile. A typical example for scintillation cameras is the uniformity variation and shift of energy spectra with rotation angle. Faster software implementations might be possible by using post-simulation modules incorporating models for those effects based on experimental measurements.

Future developments in detector design, together with faster computer systems, will improve image quality, temporal resolution, patient throughout and quantitation. It is expected that Monte Carlo calculations will enter the clinical and scientific arena and will become a method of choice to develop and to implement patient-specific dosimetry and image correction techniques and to optimize instrumentation and clinical protocols. a) Voice: 412237271 65; fax: 412237271 69; electronic mail: hzaidi@dmnu-pet5.hcuge.ch

${ }^{1}$ M. S. Rosenthal, J. Cullom, W. Hawkins, S. C. Moore, B. M. Tsui, and M. Yester, "Quantitative SPECT imaging: a review and recommendations by the Focus Committee of the Society of Nuclear Medicine Computer and Instrumentation Council," J. Nucl. Med. 36, 1489-1513 (1995).

${ }^{2}$ H. Zaidi, "Quantitative SPECT: Recent developments in detector response, attenuation and scatter correction techniques,' Phys. Medica 12, 101-117 (1996).

${ }^{3}$ D. D. McCracken, "The Monte Carlo method," Sci. Am. 192, 90-96 (1955).

${ }^{4} \mathrm{H}$. Kahn, "Use of different Monte Carlo sampling techniques," in Monte Carlo Methods, edited by H. A. Meyer (Wiley, New York, 1956).

${ }^{5}$ D. E. Raeside, "Monte Carlo principles and applications," Phys. Med. Biol. 21, 181-197 (1976).

${ }^{6}$ T. M. Jenkins, W. R. Nelson, and A. Rindi, Monte Carlo Transport of Electrons and Photons (Plenum, New York, 1988).

${ }^{7}$ R. L. Morin, Monte Carlo Simulation in the Radiological Sciences (CRC, Boca Raton, FL, 1988).

${ }^{8}$ I. Lux and L. Koblinger, Monte Carlo Particle Transport Methods: Neutron and Photon Calculations (Chemical Rubber Company, Boca Raton, FL, 1991).

${ }^{9}$ M. Ljungberg, S-E. Strand, and M. A. King, Monte Carlo Calculations in Nuclear Medicine: Applications in Diagnostic Imaging (IOP, London, 1998).

${ }^{10} \mathrm{P}$. Andreo, "Monte Carlo techniques in medical radiation physics," Phys. Med. Biol. 36, 861-920 (1991).

${ }^{11}$ J. E. Turner, H. A. Wright, and R. N. Hamm, "A Monte Carlo primer for health physicists," Health Phys. 48, 717-733 (1985).

${ }^{12}$ D. Murray, "Using EGS4 Monte Carlo in medical radiation physics," Australas. Phys. Eng. Sci. Med. 13, 132-147 (1990).

${ }^{13}$ M. M. Dresser, Scattering effects in radioisotopic imaging, Ph.D. thesis, University of Michigan, University Microfilms, Ann Arbor, MI, 1972.

${ }^{14} \mathrm{~J}$. W. Beck, "Analysis of a camera based single-photon emission computed tomography (SPECT) system," Thesis, University of Duke, University Microfilms, Ann Arbor, MI, 1982.

${ }^{15}$ M. Ljungberg and S.-E. Strand, "A Monte Carlo program for the simulation of scintillation camera characteristics," Comput. Methods Programs Biomed. 29, 257-272 (1989).

${ }^{16}$ D. J. de Vries, S. C. Moore, R. E. Zimmerman, S. P. Mueller, B. Friedland, and R. C. Lanza, "Development and validation of a Monte Carlo simulation of photon transport in an Anger camera," IEEE Trans. Med. Imaging 9, 430-438 (1990).

${ }^{17}$ I. G. Zubal, C. R. Harrell, and P. D. Esser, "Monte Carlo determination of emerging energy spectra for diagnostically realistic radiopharmaceutical distributions." Nucl. Instrum. Methods Phys. Res. A 299, 544-547 (1990).

${ }^{18}$ J. C. Yanch, A. B. Dobrzeniecki, C. Ramanathan, and R. Behrman, "Physically realistic Monte Carlo simulation of source collimator and tomographic data acquisition for emission computed tomography,' Phys. Med. Biol. 37, 853-870 (1992).

${ }^{19}$ N. A. Keller and J. R. Lupton, "PET detector ring aperture function calculations using Monte Carlo techniques," IEEE Trans. Nucl. Sci. 30, 676-680 (1983).

${ }^{20}$ C. J. Thompson, J.-M. Cantu, and Y. Picard, "PETSIM: Monte Carlo program simulation of all sensitivity and resolution parameters of cylindrical positron imaging systems," Phys. Med. Biol. 37, 731-749 (1992).

${ }^{21}$ R. L. Harrison, S. D. Vannoy, D. R. Haynor, S. B. Gillispie, M. S. Kaplan, and T. K. Lewellen, "Preliminary experience with the photon history generator module for a public-domain simulation system for emission tomography," in Conf. Rec. IEEE Med. Imag. Conf., San Francisco, 1993 (IEEE, New York, 1994), pp. 1154-1158.

${ }^{22}$ H. Zaidi, A. Herrmann Scheurer, and C. Morel, "Development of an object-oriented Monte Carlo simulator for 3D positron tomography," in Conf. Rec. of the International Meeting in Fully Three-Dimensional Image Reconstruction in Radiology and Nuclear Medicine, Nemacolin Woodlands, 1997, edited by P. Kinahan and D. Townsend (UPMC, Pittsburgh, 1997), pp. 176-179.

${ }^{23}$ M. Dahlbom, L. R. MacDonald, M. Schmand, L. Eriksson, M. Andreaco, and C. Williams, "A YSO/LSO phoswich array detector for single and coincidence photon imaging," IEEE Trans. Nucl. Sci. 45, 1128-1132 (1998). 
${ }^{24}$ F. James, "Monte Carlo theory and practice," Rep. Prog. Phys. 43, 1145-1189 (1980).

${ }^{25}$ T. Lund, "An introduction to the Monte Carlo method," Rep CERN/HS/ 067 CERN data handling division, Geneva, 1981.

${ }^{26}$ G. Marsaglia and A. Zaman, "Some portable very-long-period random number generators,"' Comput. Phys. 8, 117-121 (1994).

${ }^{27}$ J. C. Ehrhardt, “'Generation of pseudorandom numbers,'” Med. Phys. 13, 240-241 (1986).

${ }^{28}$ A. M. Ferrenberg, D. P. Landau, and Y. J. Wong, "Monte Carlo simulations: hidden errors from "good" random number generators," Phys. Rev. Lett. 69, 3382-3384 (1992).

${ }^{29}$ I. Vattulainen, K. Kankaala, J. Saarinen, and T. Ala-Nissila, " A comparative study of some pseudorandom number generators," Comput. Phys. Commun. 86, 209-226 (1995).

${ }^{30}$ T. E. Booth, "The intelligent random number technique in MCNP," Nucl. Sci. Eng. 100, 248-254 (1988).

${ }^{31}$ D. H. Lehmer, "Mathematical methods in large-scale computing units," in Proceedings of the 2nd Symposium on Large-Scale Digital Calculating Machinery, Cambridge (Massachusetts Harvard University Press, Cambridge, 1949), pp. 141-146.

${ }^{32} \mathrm{G}$. Marsaglia and A. Zaman, "Monkey tests for random number generators," Comput. Math. Appl. 23, 1-10 (1993).

${ }^{33}$ D. E. Knuth, The Art of Computer Programming, Vol. 2: Seminumerical Algorithms, 2nd ed. (Addison-Wesley Reading, MA, 1981).

${ }^{34}$ W. H. Press, Numerical Recipes in C, The Art of Scientific Computing (University Press, Cambridge, 1992).

${ }^{35} \mathrm{~S}$. L. Anderson, "Random number generators on vector supercomputers and other advanced architectures," SIAM (Soc. Ind. Appl. Math.) Rev. 32, 221-251 (1990).

${ }^{36}$ M. Mascagni, S. A. Cuccaro, D. V. Pryor, and M. L. Robinson, “A fast, high-quality, and reproducible lagged-Fibonacci pseudorandom number generator," J. Comput. Phys. 15, 211-219 (1995).

${ }^{37}$ R. P. Brent, "On the periods of generalized Fibonacci recurrences," Math. Comput. 63, 389-401 (1994).

${ }^{38} \mathrm{H}$. Zaidi, C. Labbé, and C. Morel, "Implementation of an environment for Monte Carlo simulation of fully 3D positron tomography on a highperformance parallel platform,' Parallel Comput. 24, 1523-1536 (1998).

${ }^{39}$ A. E. Nahum, Overview of Photon and Electron Monte Carlo, edited by T. Jenkins, W. Nelson, A. Rindi, A. Nahum, and D. W. Rogers (Plenum, New York, 1989), pp. 3-20.

${ }^{40}$ D. W. O. Rogers and A. F. Bielajew, "Monte Carlo techniques of electron and photon transport for radiation dosimetry," in The Dosimetry of Ionizing Radiation, edited by K. R. Kase, B. E. Bjarngard, and F. H. Attix (Academic, New York, 1990), pp. 427-539.

${ }^{41}$ M. J. Berger and J. H. Hubbell, "XCOM: Photon cross sections on a personal computer," NBSIR 87-3597, 1987.

${ }^{42}$ D. K. Trubey, M. J. Berger, and J. H. Hubbell, "Photon cross sections for ENDF/B-IV," American Nuclear Society Topical Meeting, Advances in Nuclear Engineering Computation and Radiation Shielding, Santa Fe, New Mexico, 1989.

${ }^{43}$ R. Brun, F. Bruyant, M. Maire, A. C. McPherson, and P. Zanarini, GEANT 3, CERN DD/EE/84-1, 1987.

${ }^{44}$ Y. Picard, C. J. Thompson, and S. Marrett, "Improving the precision and accuracy of Monte Carlo simulation in positron emission tomography," IEEE Trans. Nucl. Sci. 39, 1111-1116 (1992).

${ }^{45}$ J. M. Boone and A. E. Chavez, "Comparison of x-ray cross sections for diagnostic and therapeutic medical physics," Med. Phys. 23, 1997-2005 (1997).

${ }^{46} \mathrm{H}$. Zaidi, C. Labbé and C. Morel, "Improvement of the performance and accuracy of PET Monte Carlo simulations," to appear in Proceedings of the SPIE's International Symposium on Medical Imaging, Vol. 3659, edited by J. M. Boone and J. T. Dobbins, San Diego, 1999.

${ }^{47}$ ICRU, "Tissue substitutes in radiation dosimetry and measurement," Report 44 of the International Commission on Radiation Units and Measurements, Bethesda, MD, 1989.

${ }^{48}$ W. V. Prestwich, J. Nunes, C. S. Kwok, "Beta dose point kernels for radionuclides of potential use in radioimmunotherapy," J. Nucl. Med. 30, 1036-1046 (1989).

${ }^{49}$ T. E. Hui, D. R. Fisher, J. A. Kuhn, L. E. Williams, C. Nourigat, C. C. Badger, B. G. Beatty, and J. D. Beatty, "A mouse model for calculating cross-organ beta doses from yttrium-90-labeled immunoconjugates," Cancer (N.Y.) 1, 951-957 (1994).

${ }^{50}$ E. E. Furhang, C. S. Chui, K. S. Kolbert, S. M. Larson, and G. Sgouros,
"Implementation of a Monte Carlo dosimetry method for patient-specific internal emitter therapy,'’ Med. Phys. 24, 1163-1172 (1997).

${ }^{51}$ M. J. Berger, "Monte Carlo calculation of the penetration and diffusion of fast charged particles," Methods in Computational Physics, edited by B. Alder, S. Fernbach, and M. Rotenberg (Academic, New York, 1963), Vol. 1, pp. 135-215.

${ }^{52}$ P. Andreo, J. Medin, A. F. Bielajew, "Constraints of the multiplescattering theory of Moliere in Monte Carlo simulations of the transport of charged particles," Med. Phys. 20, 1315-1325 (1993).

${ }^{53}$ A. F. Bielajew and D. W. O. Rogers, "PRESTA: The Parameter reduced electron-step transport algorithm for electron Monte Carlo transport," Nucl. Instrum. Methods Phys. Res. B 18, 165-181 (1987).

${ }^{54}$ I. Kawrakow, "Improved modeling of multiple scattering in the voxel Monte Carlo model," Med. Phys. 24, 505-517 (1997).

${ }^{55}$ D. W. O. Rogers, "Low energy electron transport with EGS," Nucl. Instrum. Methods 227, 535-548 (1984).

${ }^{56}$ C. M. Ma and A. E. Nahum, "A new algorithm for EGS4 low-energy electron transport to account for the change in discrete interaction crosssection with energy,' Nucl. Instrum. Methods Phys. Res. B 72, 319-330 (1992).

${ }^{57}$ A. F. Bielajew and D. W. O. Rogers, "Variance-reduction techniques," in Monte Carlo Transport of Electrons and Photons, edited by T. Jenkins, W. Nelson, A. Rindi, A. Nahum, and D. Rogers (Plenum, New York, 1989), pp. 407-419.

${ }^{58}$ D. R. Haynor, R. L. Harrison, and T. K. Lewellen, "Improving the efficiency of emission tomography using variance reduction techniques," IEEE Trans. Nucl. Sci. 37, 749-753 (1990).

${ }^{59}$ I. G. Zubal and C. R. Harell, "Voxel-based Monte Carlo calculations of nuclear medicine images and applied variance reduction techniques," Image Vis. Comput. 10, 342-348 (1992).

${ }^{60}$ D. R. Haynor, R. L. Harrison, and T. K. Lewellen, "The use of importance sampling techniques to improve the efficiency of photon tracking in emission tomography simulations," Med. Phys. 18, 990-1001 (1991).

${ }^{61}$ C. M. Ma and A. E. Nahum, "Calculation of absorbed dose ratios using correlated Monte Carlo sampling," Med. Phys. 20, 1189-1199 (1993).

${ }^{62}$ M. A. Holmes, T. R. Mackie, W. Sohn, P. J. Reckwerdt, T. J. Kinsella, A. F. Bielajew, and D. W. Rogers, "The application of correlated sampling to the computation of electron beam dose distributions in heterogeneous phantoms using the Monte Carlo method," Phys. Med. Biol. 38, 675-688 (1993).

${ }^{63}$ H. O. Anger, "Scintillation camera with multichannel collimators," J. Nucl. Med. 5, 515-531 (1964).

${ }^{64}$ R. J. Jaszczak, K. L. Greer, and R. E. Coleman, "SPECT using a specially designed cone beam collimator," J. Nucl. Med. 29, 1398-1405 (1988).

${ }^{65}$ B. M. W. Tsui, G. T. Gullberg, E. R. Edgerton, D. R. Gilland, J. R. Perry, and W. H. McCartney, "Design and clinical utility of a fan beam collimator for SPECT imaging of the head," J. Nucl. Med. 27, 810-819 (1986).

${ }^{66} \mathrm{D}$. A. Weber et al., "Pinhole SPECT: an approach to in vivo high resolution SPECT imaging in small laboratory animals," J. Nucl. Med. 35, 342-348 (1994).

${ }^{67} \mathrm{H}$. Zaidi, "Assessment of thyroid volume with pinhole emission computed tomography," Phys. Medica 12, 97-100 (1996).

${ }^{68} \mathrm{C}$. Miller, L. Filipow, and S. Jackson, "A review of activity quantification by planar imaging methods," J. Nucl. Sci. Technol. 23, 3-11 (1995).

${ }^{69} \mathrm{~S}$. R. Cherry, "Recent advances in instrumentation for positron emission tomography," Nucl. Instrum. Methods Phys. Res. A 348, 577-582 (1994).

${ }^{70}$ C. Michel, A. Bol, T. Spinks, D. W. Townsend, D. Bailey, S. Grootoonk, and T. Jones, "Assessment of response function in two PET scanners with and without interplane septa," IEEE Trans. Med. Imaging 10, 240248 (1991).

${ }^{71}$ M. Dahlbom, G. Rosenquist, L. Eriksson, and C. Bohm, "A study of the possibility of using multi-slice PET systems for 3D imaging," IEEE Trans. Nucl. Sci. 36, 1066-1071 (1989).

${ }^{72}$ J. M. Ollinger, "Model-based scatter correction for fully 3D PET,', Phys. Med. Biol. 41, 153-176 (1996).

${ }^{73}$ C. C. Watson, D. Newport, M. E. Casey, A. deKemp, R. S. Beanlands, and M. Schmand, "Evaluation of simulation-based scatter correction for 3-D PET cardiac imaging," IEEE Trans. Nucl. Sci. 44, 90-97 (1997).

${ }^{74}$ M. E. Daube-Witherspoon and G. Muehllehner, "Treatment of axial data in three-dimensional PET,’ J. Nucl. Med. 82, 1717-1724 (1987). 
${ }^{75}$ P. E. Kinahan and J. G. Rogers, “Analytic 3D image reconstruction using all detected events," IEEE Trans. Nucl. Sci. 36, 964-968 (1989).

${ }^{76}$ C. Comtat, C. Morel, M. Defrise, and D. W. Townsend, "The Favor algorithm for 3D PET data and its implementation using a network of transputers,' Phys. Med. Biol. 38, 929-944 (1993).

${ }^{77}$ J. T. Kuikka, K. E. Britton, V. U. Chengazi, and S. Savolainen, "Future developments in nuclear medicine instrumentation: A review," Nucl. Med. Commun. 19, 3-12 (1998).

${ }^{78} \mathrm{C}$. D. Zerby, A Monte Carlo calculation of the response of gamma-ray scintillation counters. Methods in Computational Physics, edited by B. Alder, S. Fermbach, and M. Rotenberg (Academic, New York, 1963), Vol. 1, pp. 89-134.

${ }^{79}$ M. J. Berger and S. M. Seltzer, "Response functions for sodium iodide scintillation detectors," Nucl. Instrum. Methods Phys. Res. A 104, $317-$ 332 (1972).

${ }^{80}$ D. W. O. Rogers, "More realistic Monte Carlo calculations of photon detector response functions," Nucl. Instrum. Methods 199, 531-548 (1982).

${ }^{81}$ K. Saito and S. Moriuchi, "Monte Carlo calculation of $\mathrm{NaI}(\mathrm{Tl})$ detector response functions for low-energy gamma rays," Nucl. Instrum. Methods 226, 449-454 (1984).

${ }^{82}$ H. Chan, C. Chen, K. Doi, T. R. Fewell, and R. E. Shuping, “Investigation of energy responses of germanium detectors and correction of measured spectra by means of Monte Carlo simulation,' Radiat. Res. 99, 443-463 (1984).

${ }^{83}$ H. H. Hsu, E. J. Dowdy, G. P. Estes, M. C. Lucas, J. M. Mack, C. E. Moss, and M. E. Hamm, "Efficiency of bimsuth germanate scintillators: Comparison of Monte Carlo calculations with measurements," IEEE Trans. Nucl. Sci. 37, 390-395 (1989).

${ }^{84}$ F. H. Fahey, R. E. Zimmerman, P. F. Judy, and R. C. Lanza, "'Detection efficiency of a high-pressure gas scintillation proportional chamber," Med. Phys. 14, 115-23 (1987).

${ }^{85}$ E. Tanaka, N. Nohara, and H. Murayama, "New Anger scintillation cameras with improved count rate capability," Radioisotopes 29, 320-325 (1980).

${ }^{86}$ M. Conti, A. Del Guerra, D. Mazzei, P. Russo, W. Bencivelli, E. Bartolucci, A. Messineo, V. Rosso, A. Stefanini, U. Bottigli, P. Randaccio, and W. R. Nelson, "Use of the EGS4 Monte Carlo code to evaluate the response of $\mathrm{HgI}$ and $\mathrm{CdTe}$ detectors for photons in the diagnostic energy range," Nucl. Instrum. Methods Phys. Res. A 322, 591-595 (1992).

${ }^{87}$ S. E. Derenzo, "Monte Carlo calculations of the detection efficiency of $\mathrm{NaI}(\mathrm{Tl}), \mathrm{BGO}, \mathrm{CsF} \mathrm{Ge}$ and plastic detectors for $511 \mathrm{keV}$ photons" $\mathbf{2 8}$, 11-136 (1981).

${ }^{88}$ S. E. Derenzo and J. K. Riles, "Monte Carlo calculations of the optical coupling between bismuth germanate crystals and photomultiplier tubes" 29, 191-195 (1982)

${ }^{89}$ U. Bottigli, R. Guzzardi, M. Mey, R. Bellazzini, P. Giannetti, M. A. Giorgi, M. M. Massai, and G. Tonelli, "Monte Carlo simulation and experimental tests on $\mathrm{BGO}, \mathrm{CsF}$ and $\mathrm{NaI}(\mathrm{Tl})$ crystals for positron emission tomography," J. Nucl. Med. Allied Sci. 29, 221-227 (1985).

${ }^{90}$ A. N. Bice, T. K. Lewellen, R. S. Miyaoka, R. L. Harrison, D. R. Haynor, K. R. Pollard, C. P. Hanson, and S. B. Gillispie, "Monte Carlo simulation of $\mathrm{BaF}_{2}$ detectors used in time-of-flight positron emission tomography," IEEE Trans. Nucl. Sci. 37, 696-701 (1990).

${ }^{91}$ M. I. Lopes, V. Chepel, J. C. Carvalho, R. Ferreira Marques, and A. J. P. L. Policarpo, "Performance analysis based on a Monte Carlo simulation of a liquid Xenon PET detector,' IEEE Trans. Nucl. Sci. 42, 2298-2302 (1995).

${ }^{92}$ P. F. Binkley, "'Optimization of scintillation detector timing systems using Monte Carlo analysis," IEEE Trans. Nucl. Sci. 41, 386-393 (1994).

${ }^{93}$ R. H. Huesman, E. M. Salmeron, and J. R. Baker, "Compensation for crystal penetration in high resolution positron tomography," IEEE Trans. Nucl. Sci. 36, 1100-1107 (1989).

${ }^{94}$ K. A. Comanor, P. R. G. Virador, and W. W. Moses, "Algorithms to identify detector Compton scatter in PET modules,' IEEE Trans. Nucl. Sci. 43, 2213-2218 (1996)

${ }^{95}$ T. A. DeVol, W. W. Moses, and S. E. Derenzo, “Monte Carlo optimization of depth-of-interaction resolution in PET crystals," IEEE Trans. Nucl. Sci. 40, 170-174 (1993).

${ }^{96}$ W. W. Moses and S. E. Derenzo, "Design studies for a PET detector module using a PIN photodiode to measure depth of interaction," IEEE Trans. Nucl. Sci. 41, 1441-1445 (1994).

${ }^{97}$ G. Tsang, C. Moisan, and J. G. Rogers, A simulation to model position encoding multicrystal PET detectors, IEEE Trans. Nucl. Sci. 42, 22362243 (1995).

${ }^{98}$ C. Moisan, J. G. Rogers, K. R. Buckley, T. J. Ruth, M. W. Stazyk, and G. Tsang "Design studies of a depth-encoding large aperture PET camera," IEEE Trans. Nucl. Sci. 42, 1041-1050 (1995).

${ }^{99}$ D. Vozza, C. Moisan, and S. Paquet, "An improved model for energy resolution of multicrystal encoding detectors for PET," IEEE Trans. Nucl. Sci. 44, 179-183 (1997).

${ }^{100}$ S. Delorme, R. Frei, C. Jospeh, J.-F. Loude, and C. Morel, "Use of a neural network to exploit light division in a triangular scintillating crystal,' Nucl. Instrum. Methods Phys. Res. A 373, 111-118 (1996).

${ }^{101}$ D. W. Litzenberg, F. D. Becchetti, and D. A. Roberts, "On-line PET monitoring of radiotherapy beams: image reconstruction and Monte Carlo simulations of detector geometries," IEEE Trans. Nucl. Sci. 44, 16461657 (1997).

${ }^{102}$ A. G. Schulz, L. G. Knowles, L. C. Kohlenstein, R. F. Mucci, and W. A. Yates "Quantitative assessment of scanning-system parameters,"' J. Nucl. Med. 11, 61-68 (1970).

${ }^{103}$ R. E. A. Dye, "Simulation of clinical scitingrams for nuclear medicine imaging devices," Phys. Med. Biol. 33, 1329-1334 (1988).

${ }^{104}$ P. Gantet, J. P. Esquerre, B. Danet, and R. Guiraud, "A simulation method for studying scintillation camera collimators," Phys. Med. Biol. 35, 659-669 (1990).

${ }^{105} \mathrm{H}$. Zaidi, "Comparative methods for quantifying thyroid volume using planar imaging and SPECT,'” J. Nucl. Med. 37, 1421-1426 (1996).

${ }^{106}$ L. J. Hahn, R. J. Jaszczak, G. T. Gullberg, C. E. Floyd, S. H. Manglos, K. L. Greer, and R. E. Coleman, "Noise characteristics for cone beam collimators: a comparison with parallel hole collimator," Phys. Med. Biol. 33, 541-555 (1988).

${ }^{107}$ S. Kimiaei and S. A. Larsson, "Optimal design of planar-concave collimators for SPECT—an analytical approach," Phys. Med. Biol. 43, 637650 (1998).

${ }^{108}$ J. J. Battista and M. J. Bronskill, "Compton-scatter tissue densitometry: calculation of single and multiple scatter photon fluences," Phys. Med. Biol. 23, 1-23 (1978).

${ }^{109}$ S. Webb, D. M. Binnie, M. A. Flower, and R. J. Ott, "Monte Carlo modelling of the performance of a rotating slit-collimator for improved planar gamma-camera imaging,"' Phys. Med. Biol. 37, 1095-1108 (1992).

${ }^{110}$ C. E. Metz, F. B. Atkins, and R. N. Beck, “The geometric transfer function component for scintillation camera collimators with straight parallel holes,' Phys. Med. Biol. 25, 1059-1070 (1980).

${ }^{111}$ S. Kimiaei, M. Ljungberg, and S. A. Larsson, "Evaluation of optimally designed planar-concave collimators in single-photon emission tomography,' Eur. J. Nucl. Med. 24, 1398-1404 (1997).

${ }^{112} \mathrm{C}$. J. Thompson, "The effect of collimation on singles rates in multi-slice PET," IEEE Trans. Nucl. Sci. 35, 598-602 (1988)

${ }^{113}$ W. M. Digby, M. Dahlbom, and E. J. Hoffman, "Detector, shielding and geometric design factors for a high-resolution PET system,' IEEE Trans. Nucl. Sci. 37, 664-670 (1990).

${ }^{114}$ S. C. Moore, D. J. de Vries, B. Nandram, M. F. Kijewski, and S. P. Mueller, "Collimator optimization for lesion detection incorporating prior information about lesion size," Med. Phys. 22, 703-713 (1995).

${ }^{115}$ R. J. Jaszczak, C. E. Floyd, S. H. Manglos, K. L. Greer, and R. E. Coleman, "Cone beam collimation for single photon emission computed tomography: analysis, simulation, and image reconstruction using filtered backprojection," Med. Phys. 13, 484-489 (1986).

${ }^{116}$ K. R. Pollard, A. N. Bice, J. F. Eary, L. D. Durack, and T. K. Lewellen, "A method for imaging therapeutic doses of iodine-131 with a clinical gamma camera," J. Nucl. Med. 33, 771-776 (1992).

${ }^{117}$ H. Wang, R. J. Jaszczak, and R. E. Coleman, "Monte Carlo modeling of penetration effect for iodine-131 pinhole imaging," IEEE Trans. Nucl. Sci. 43, 3272-3277 (1996).

${ }^{118}$ M. F. Smith and R. J. Jaszczak, "The effect of gamma ray penetration on angle-dependent sensitivity for pinhole collimation in nuclear medicine," Med. Phys. 24, 1701-1709 (1997).

${ }^{119}$ C. J. Thompson, J. M. Roney, R. Lecomte, D. Schmitt, and L. R. Lupton, "Dependence of the coincidence aperture function of narrow BGO crystals on crystal shape and light encoding schemes," Phys. Med. Biol. 31, 491-506 (1986).

${ }^{120} \mathrm{C}$. J. Thompson, "The effect of collimation on scatter fraction in multislice PET," IEEE Trans. Nucl. Sci. 35, 598-602 (1988).

${ }^{121}$ J. Bradshaw, C. Burnham, and J. Correia, “Application of Monte Carlo 
methods to the design of SPECT detector systems," IEEE Trans. Nucl. Sci. 32, 753-757 (1985).

${ }^{122}$ L. R. Lupton and N. A. Keller, "Performance study of single-slice positron emission tomography scanners by Monte Carlo techniques," IEEE Trans. Med. Imaging 2, 154-168 (1983).

${ }^{123}$ J. A. Heanue, J. K. Brown, H. R. Tang, and B. H. Hasegawa, "A bound on the energy resolution required for quantitative SPECT,' Med. Phys. 23, 169-173 (1996).

${ }^{124}$ G. H. Kramer, M. J. Chamberlain, and S. Yiu, "A study of thyroid radioiodine monitoring by Monte Carlo simulations: implications for equipment design," Phys. Med. Biol. 42, 2175-2182 (1997).

${ }^{125}$ C. W. Stearns, C. A. Burnham, D. A. Chesler, and G. L. Brownell, "Simulation studies for cylindrical positron tomography," IEEE Trans. Nucl. Sci. 35, 708-711 (1988).

${ }^{126}$ J. G. Rogers, R. Harrop, P. E. Kinahan, N. A. Wilkinson, and G. H. Coombes, "Conceptual design of a whole body PET machine," IEEE Trans. Nucl. Sci. 35, 680-684 (1988).

${ }^{127}$ A. Del Guerra and W. R. Nelson, Positron Emission Tomography Applications of EGS, in Monte Carlo Transport of Electrons and Photons, edited by T. M. Jenkins, W. R. Nelson, and A. Rindi (Plenum, New York, 1988) pp. 469-484.

${ }^{128}$ D. Bollini, A. Del Guerra, G. Di Domenico, M. Galli, M. Gambaccini, and G. Zavattini, "Sub-millimeter planar imaging with positron emitters: EGS4 code simulation and experimental results," IEEE Trans. Nucl. Sci. 44, 1499-1502 (1997).

${ }^{129} \mathrm{C}$. J. Thompson, "The effects of detector material and structure on PET spatial resolution and efficiency,"' IEEE Trans. Nucl. Sci. 37, 718-724 (1990).

${ }^{130} \mathrm{G}$. Tzanakos and S. Pavlopoulos, "Development and validation of a simulation model for the design of a PET scanner," IEEE Trans. Nucl. Sci. 39, 1093-1098 (1992).

${ }^{131}$ S. Pavlopoulos and G. Tzanakos, "Design and performance evaluation of a high-resolution small animal positron tomograph," IEEE Trans. Nucl. Sci. 43, 3249-3255 (1996).

${ }^{132}$ R. S. Miyaoka, "Dynamic high resolution positron emission imaging of rats," Biomed. Sci. Instrum. 27, 35-42 (1991).

${ }^{133}$ W. W. Moses, P. R. G. Virador, S. E. Derenzo, R. H. Huesman, and T. F. Budinger, "Design of a high-resolution, high-sensitivity PET camera for human brains and small animals,' IEEE Trans. Nucl. Sci. 44, 1487-1491 (1997).

${ }^{134}$ J. G. Rogers, M. Stazyk, R. Harrop, C. J. Dykstra, J. S. Barney, M. S. Atkins, and P. E. Kinahan, "Towards the design of a positron imaging camera"' IEEE Trans. Nucl. Sci. 37, 789-794 (1990).

${ }^{135}$ H. Zaidi, A. Herrmann Scheurer, and C. Morel, “An object-oriented Monte Carlo simulator for 3D positron tomographs," Comput. Methods Programs Biomed. 58, 133-145 (1999).

${ }^{136}$ M. Defrise, "A factorization method for the 3D X-ray transform," Inverse Probl. 11, 983-994 (1995).

${ }^{137}$ K. M. Hanson, "Method of evaluating image-recovery algorithms based on task performance," J. Opt. Soc. Am. A 7, 1294-1304 (1990).

${ }^{138}$ T. A. Riauka, H. R. Hooper, and Z. W. Gortel, "Experimental and numerical investigation of the 3D SPECT photon detection kernel for nonuniform attenuating media,' Phys. Med. Biol. 41, 1167-1189 (1996).

${ }^{139}$ M. F. Smith, C. E. Floyd, R. J. Jaszczak, and R. E. Coleman, "Threedimensional photon detection kernels and their application to SPECT reconstruction," Phys. Med. Biol. 3, 605-622 (1992).

${ }^{140}$ M. A. King, W. Xia, D. J. de Vries, T. S. Pan, B. J. Villegas, S. Dahlberg, B. M. Tsui, M. Ljungberg, and H. T. Morgan, "A Monte Carlo investigation of artifacts caused by liver uptake in single-photon emission computed tomography perfusion imaging with technetium 99m-labeled agents,'” J. Nucl. Cardiol. 3, 18-29 (1996).

${ }^{141}$ C. E. Floyd, R. J. Jaszczak, and R. E. Coleman, "Convergence of the maximum likelihood reconstruction algorithm for emission computed tomography," Phys. Med. Biol. 32, 463-476 (1987).

${ }^{142}$ J. E. Bowsher and C. E. Floyd, "Treatment of Compton scattering in maximum-likelihood, expectation-maximization reconstructions of SPECT images,"' J. Nucl. Med. 32, 1285-1291 (1991).

${ }^{143}$ Z. Liang, R. J. Jaszczak, C. E. Floyd, K. L. Greer, and R. E. Coleman, "Bayesian reconstruction for SPECT: validation with Monte Carlo simulation, experimental phantom, and real patient data,' Int. J. Imaging Syst. Technol. 1, 149-169 (1989).

${ }^{144}$ D. M. Higdon, J. E. Bowsher, V. E. Johnson, T. G. Turkington, D. R. Gilland, and R. J. Jaszczak, "Fully Bayesian estimation of Gibbs hyper- parameters for emission computed tomography data,' IEEE Trans. Med. Imaging 16, 516-526 (1997).

${ }^{145}$ D. S. Lalush and B. M. W. Tsui, "Fast and stable maximum a-Posteriori conjugate gradient reconstruction algorithm," Med. Phys. 22, 1273-1284 (1995).

${ }^{146}$ G. Chinn and S. C. Huang, "A general class of preconditioners for statistical iterative reconstruction of emission computed tomography," IEEE Trans. Med. Imaging 16, 1-10 (1997).

${ }^{147}$ C. E. Floyd, R. J. Jaszczak, and R. E. Coleman, "Inverse Monte Carlo: a unified reconstruction algorithm for SPECT," IEEE Trans. Nucl. Sci. 32, 779-785 (1985).

${ }^{148}$ W. L. Dunn, "Inverse Monte Carlo analysis," J. Comput. Phys. 41, 154166 (1981).

${ }^{149}$ C. E. Floyd, R. J. Jaszczak, K. L. Greer, and R. E. Coleman, "Inverse Monte Carlo as a unified reconstruction algorithm for ECT," J. Nucl. Med. 27, 1577-1585 (1986).

${ }^{150}$ C. E. Floyd, R. J. Jaszczak, S. H. Manglos, and R. E. Coleman, "Compensation for collimator divergence in SPECT using inverse Monte Carlo reconstruction," IEEE Trans. Nucl. Sci. 35, 784-787 (1988).

${ }^{151}$ D. W. Wilson, B. M. W. Tsui, and H. Barrett, "Noise properties of the EM algorithm: II. Monte Carlo simulations,” Phys. Med. Biol. 39, 847871 (1994).

${ }^{152}$ W. Wang and G. Gindi, "Noise analysis of MAP-EM algorithms for emission tomography,"' Phys. Med. Biol. 42, 2215-2232 (1997).

${ }^{153}$ C. E. Floyd, R. J. Jaszczak, C. C. Harris, and R. E. Coleman, "Energy and spatial distribution of multiple order Compton scatter in SPECT: A Monte Carlo investigation," Phys. Med. Biol. 29, 1217-1230 (1984).

${ }^{154}$ C. E. Floyd, R. J. Jaszczak, and R. E. Coleman, "Scatter detection in SPECT imaging: dependence on source depth, energy, and energy window," Phys. Med. Biol. 33, 1075-1081 (1988).

${ }^{155}$ T. S. Pan, M. A. King, D. J. de Vries, and M. Ljungberg, "'Segmentation of the body and lungs from Compton scatter and photopeak window data in SPECT: A Monte-Carlo investigation," IEEE Trans. Med. Imaging 15, 13-24 (1996).

${ }^{156} \mathrm{H}$. Zaidi, "Organ volume estimation using SPECT," IEEE Trans. Nucl. Sci. 43, 2174-2182 (1996).

${ }^{157} \mathrm{C}$. A. Lowry and M. J. Cooper, "The problem of Compton scattering in emission tomography: a measurement of its spatial distribution" Phys. Med. Biol. 32, 1187-1191 (1987).

${ }^{158}$ A. Kojima, M. Matsumoto, M. Takahashi, and S. Uehara, "Effect of energy resolution on scatter fraction in scintigraphic imaging: Monte Carlo study,"' Med. Phys. 20, 1107-1113 (1993).

${ }^{159}$ S. H. Manglos, C. E. Floyd, R. J. Jaszczak, K. L. Greer, C. C. Harris, and R. E. Coleman, "Experimentally measured scatter fractions and energy spectra as a test of Monte Carlo simulations,' Phys. Med. Biol. 32, 335343 (1987).

${ }^{160}$ F. J. Beekman and M. A. Viergever, "Fast SPECT simulation including object shape dependent scatter," IEEE Trans. Med. Imaging 14, 271-282 (1995).

${ }^{161}$ J. S. Fleming, "Evaluation of a technique for simulation of gamma camera images," Phys. Med. Biol. 41, 1855-1861 (1996).

${ }^{162}$ D. Gagnon, L. Laperriere, N. Pouliot, D. J. de Vries, and S. C. Moore, "Monte Carlo analysis of camera-induced spectral contamination for different primary energies," Phys. Med. Biol. 37, 1725-1739 (1992).

${ }^{163}$ E. C. Frey and B. M. W. Tsui, "Parameterization of the scatter response function in SPECT imaging using Monte Carlo simulation," IEEE Trans. Nucl. Sci. 37, 1308-1315 (1990).

${ }^{164}$ K. F. Koral, X. Wang, K. R. Zasadny, N. H. Clinthorne, W. L. Rogers, C. E. Floyd, and R. J. Jaszczak, "Testing of local gamma-ray scatter fractions determined by spectral fitting," Phys. Med. Biol. 36, 177-190 (1991).

${ }^{165}$ M. S. Rosenthal and L. A. Henry, "Monte Carlo simulation of scatter in non-uniform symmetrical attenuating media for point and distributed sources," Appl. Radiat. Isot. 43, 449-454 (1992).

${ }^{166} \mathrm{E}$. C. Frey and B. M. W. Tsui, "Modeling the scatter response function in inhomogeneous scattering media for SPECT," IEEE Trans. Nucl. Sci. 41, 1585-1593 (1994).

${ }^{167}$ E. C. Frey and B. M. W. Tsui, "A fast projector-backprojector pair modeling the asymmetric, spatially varying scatter response function for scatter compensation in SPECT imaging," IEEE Trans. Nucl. Sci. 40, 1192-1197 (1993).

${ }^{168}$ M. Ljungberg, S.-E. Strand, N. Rajeevan, and M. A. King, "Monte Carlo simulation of transmission studies using a planar source with a parallel 
collimator and a line source with a fan-beam collimator,' IEEE Trans. Nucl. Sci. 41, 1577-1584 (1994).

${ }^{169}$ K. Kawamura, K. Ogawa, A. Kubo, and T. Ichihara, "Quantitative measurement of scattered photons during gamma ray transmission CT using Monte Carlo simulation,' IEEE Trans. Nucl. Sci. 44, 1225-1230 (1997).

${ }^{170}$ R. D. Speller and J. A. Horrocks, "A Monte Carlo study of multiple scatter effects in Compton scatter densitometry," Med. Phys. 15, 707712 (1988).

${ }^{171}$ C. E. Floyd, R. J. Jaszczak, C. C. Harris, K. L. Greer, and R. E. Coleman, " Monte Carlo evaluation of Compton scatter subtraction in single photon emission computed tomography,', Med. Phys. 12, 776-778 (1985).

${ }^{172}$ M. C. Gilardi, V. Bettinardi, A. Todd-Pokropek, L. Milanesi, and F. Fazio, "Assessment and comparison of three scatter correction techniques in single photon emission computed tomography,', J. Nucl. Med. 29, 1971-1979 (1988).

${ }^{173}$ J. Mas, P. Hannequin, R. Ben Younes, B. Bellaton, and R. Bidet, “'Scatter correction in planar imaging and SPECT by constrained factor analysis of dynamic structures (FADS),'” Phys. Med. Biol. 35, 1451-1465 (1990).

${ }^{174}$ M. Ljungberg and S.-E. Strand, "Attenuation correction in SPECT based on transmission studies and Monte Carlo simulations of build-up functions,' J. Nucl. Med. 31, 493-500 (1990).

${ }^{175}$ C. Miller, L. Filipow, S. Jackson, and T. Riauka "Planar imaging quantification using 3D attenuation correction data and Monte Carlo simulated buildup factors,', Phys. Med. Biol. 41, 1401-1423 (1996).

${ }^{176}$ J. Q. Luo, K. F. Koral, M. Ljungberg, C. E. Floyd, and R. J. Jaszczak, “A Monte Carlo investigation of dual-energy-window scatter correction for volume-of-interest quantification in ${ }^{99 \mathrm{~m}}$ Tc SPECT,', Phys. Med. Biol. 40, 181-199 (1995).

${ }^{177}$ M. Ljungberg and S.-E. Strand, "Scatter and attenuation correction in SPECT using density maps and Monte Carlo simulated scatter functions,'’ J. Nucl. Med. 31, 1560-1567 (1990).

${ }^{178}$ M. Ljungberg and S.-E. Strand, "Attenuation and scatter correction in SPECT for sources in a nonhomogeneous object: A Monte Carlo study,', J. Nucl. Med. 32, 1278-1284 (1991).

${ }^{179}$ H. Naude, A. van Aswegen, C. P. Herbst, M. G. Lotter, and P. H. Pretorius, "A Monte Carlo evaluation of the channel ratio scatter correction method,' Phys. Med. Biol. 41, 1059-1066 (1996).

${ }^{180}$ A. Welch, G. T. Gullberg, P. E. Christian, F. L. Datz, and H. T. Morgan, "A transmission-map-based scatter correction technique for SPECT in inhomogeneous media,', Med. Phys. 22, 1627-1635 (1995).

${ }^{181}$ M. S. Kaplan, R. S. Miyaoka, S. K. Kohlmyer, D. R. Haynor, R. L. Harrison, and T. K. Lewellen, "Scatter and attenuation correction for ${ }^{111}$ In based on energy spectrum fitting,', Med. Phys. 23, 1277-1285 (1996).

${ }^{182}$ G. S. Hademenos, M. A. King, M. Ljungberg, I. G. Zubal, and C. R. Harrell, "A scatter correction method for T1-201 images: A Monte Carlo investigation,', IEEE Trans. Nucl. Sci. 40, 1179-1186 (1993).

${ }^{183} \mathrm{~K}$. Ogawa, "Simulation study of triple-energy-window scatter correction in combined T1-201, Tc-99m SPECT,' Ann. Nucl. Med. 8, 277-281 (1994).

${ }^{184}$ G. J. Hademenos, M. Dahlbom, and E. J. Hoffman, "Simultaneous dualisotope technetium-99m/thallium-201 cardiac SPECT imaging using a projection-dependent spilldown correction factor,' Eur. J. Nucl. Med. 22, 465-472 (1995).

${ }^{185}$ M. Ljungberg, P. Msaki, and S-E. Strand, “Comparison of dual-window and convolution scatter correction techniques using the Monte Carlo method,', Phys. Med. Biol. 35, 1099-1110 (1990).

${ }^{186}$ M. Ljungberg, M. A. King, G. J. Hademenos, and S. E. Strand, "Comparison of four scatter correction methods using Monte Carlo simulated source distributions,' J. Nucl. Med. 35, 143-151 (1994).

${ }^{187}$ I. Buvat, M. Rodriguez-Villafuerte, A. Todd-Pokropek, H. Benali, and R. Di Paola "Comparative assessment of nine scatter correction methods based on spectral analysis using Monte Carlo simulations,', J. Nucl. Med. 36, 1476-1488 (1995).

${ }^{188}$ Y. Narita, S. Eberl, H. Iida, B. F. Hutton, M. Braun, T. Nakamura, and G. Bautovich, "Monte Carlo and experimental evaluation of accuracy and noise properties of two scatter correction methods for SPECT,' Phys. Med. Biol. 41, 2481-2496 (1996).

${ }^{189}$ J. Logan and H. J. Bernstein, “A Monte Carlo simulation of Compton scattering in positron emission tomography,' J. Comput. Assist. Tomogr. 7, 316-320 (1983).

${ }^{190}$ D. Acchiappati, N. Cerullo, and R. Guzzardi, “Assessment of the scatter fraction evaluation methodology using Monte Carlo simulation techniques,'” Eur. J. Nucl. Med. 15, 683-686 (1989).

${ }^{191}$ C. Moisan, P. Tupper, J. G. Rogers, and J. K. de Jong, "A Monte Carlo study of the acceptance to scattered events in a depth encoding PET camera," IEEE Trans. Nucl. Sci. 43, 1974-2019 (1996).

${ }^{192}$ J. S. Barney, J. G. Rogers, R. Harrop, and H. Hoverath, “'Object shape dependent scatter simulations for PET," IEEE Trans. Nucl. Sci. 38, 719725 (1991).

${ }^{193}$ L. E. Adam, M. E. Bellemann, G. Brix, and W. J. Lorenz, "Monte Carlobased analysis of PET scatter components," J. Nucl. Med. 37, 20242029 (1996).

${ }^{194}$ S. I. Ziegler and W. K. Kuebler, "Monte Carlo simulation of the scatter component in small animal positron volume-imaging devices," Z. Med. Phys. 3, 83-87 (1993).

${ }^{195}$ C. S. Levin, M. Dahlbom, and E. J. Hoffman, "A Monte Carlo correction for the effect of Compton scattering in 3-D PET brain imaging," IEEE Trans. Nucl. Sci. 42, 1181-1188 (1995).

${ }^{196}$ D. R. Haynor, R. L. Harrison, and T. K. Lewellen, "Energy-based scatter correction for 3D PET: A Monte Carlo study of best possible results," in Ref. 22, pp. 52-54.

${ }^{197}$ M. J. Berger, "MIRD pamphlet 2: energy deposition in water by photons from point isotropic sources," J. Nucl. Med. 9, 15-25 (1968).

${ }^{198}$ M. J. Berger, "MIRD pamphlet 7: distribution of absorbed doses around point sources of electrons and beta particles in water and other media," J. Nucl. Med. 12, 5-23 (1971).

${ }^{199}$ W. Snyder, M. R. Ford, and G. Warner, "Estimates of specific absorbed fractions for photon sources uniformly distributed in various organs of a heterogeneous phantom. NM/MIRD Pamphlet No. 5, Society of Nuclear Medicine Publication, New York, 1978.

${ }^{200} \mathrm{M}$. Cristy, "Applying the reciprocal dose principle to heterogeneous phantoms: practical experience from Monte Carlo studies," Phys. Med. Biol. 28, 1289-1303 (1983).

${ }^{201}$ A. Aissi and J. W. Poston, "Comparison of measured and calculated internal absorbed doses in a heterogeneous phantom,' Phys. Med. Biol. 32, 1245-1256 (1987).

${ }^{202}$ M. Cristy and K. F. Eckerman, "'Specific absorbed fractions of energy at various ages from internal photon sources," Report ORNL/TM 8381/V1V7, Oak Ridge, TN, 1987.

${ }^{203}$ J. W. Poston, K. A. Kodimer, and W. E. Bolch, "A revised model for the calculation of absorbed energy in the gastrointestinal tract," Health Phys. 71, 307-314 (1996).

${ }^{204}$ A. N. Bice, J. M. Links, D. F. Wong, and H. N. Wagner, "Absorbed fractions for dose calculations of neuroreceptor PET studies," Eur. J. Nucl. Med. 11, 127-131 (1985).

${ }^{205}$ P. K. Leichner and C. S. Kwok, "Tumor dosimetry in radioimmunotherapy: methods of calculation for beta particles," Med. Phys. 20, 529534 (1993).

${ }^{206} \mathrm{G}$. Akabani, J. W. Poston, and W. E. Bolch, "Estimates of beta absorbed fractions in small tissue volumes for selected radionuclides," J. Nucl. Med. 32, 835-839 (1991).

${ }^{207}$ J. C. Johnson, S. M. Langhorst, S. K. Loyalka, W. A. Volkert, and A. R. Ketring, "Calculation of radiation dose at a bone to marrow interface using Monte Carlo modeling techniques (EGS4)," J. Nucl. Med. 33, 623628 (1992)

${ }^{208}$ C. S. Kwok, P. J. Bialobzyski, S. K. Yu, and W. V. Prestwich, "Effect of tissue inhomogeneity on dose distribution of point sources of low-energy electrons,"' Med. Phys. 17, 786-793 (1990).

${ }^{209}$ C. S. Kwok, P. J. Bialobzyski, S. K. Yu, "Effect of tissue inhomogeneity on dose distribution of continuous activity of low-energy electrons in bone marrow cavities with different topologies," Med. Phys. 18, 533541 (1991).

${ }^{210}$ B. L. Werner, C. S. Kwok, and I. J. Das, "Dose distributions in regions containing beta sources: large spherical source regions in a homogeneous medium," Med. Phys. 15, 358-363 (1988).

${ }^{211}$ B. L. Werner, M. Rahman, W. N. Salk, and C. S. Kwok, "Dose distributions in regions containing beta sources: uniform spherical source regions in homogeneous media," Med. Phys. 18, 1181-1191 (1991).

${ }^{212}$ T. R. Mackie, A. F. Bielajew, D. W. Rogers, and J. J. Battista, "Generation of photon energy deposition kernels using the EGS Monte Carlo code,' Phys. Med. Biol. 33, 1-20 (1988).

${ }^{213}$ G. Sgouros, S. Chiu, K. S. Pentlow, L. J. Brewster, H. Kalaigian, B. Baldwin, F. Daghighian, M. C. Graham, S. M. Larson, and R. Mohan, 
"Three-dimensional dosimetry for radioimmunotherapy treatment planning,', J. Nucl. Med. 34, 1595-1601 (1993).

${ }^{214} \mathrm{M}$. J. Berger, "Improved point kernel for electrons and Beta-ray dosimetry,"' Report NBSIR, 1973, pp. 73-107.

${ }^{215}$ W. G. Cross, N. O. Freedman, and P. Y. Wong, "Beta-ray dose distributions from point sources in an infinite water medium," Health Phys. 63, 160-171 (1992).

${ }^{216}$ D. J. Simpkin and T. R. Mackie "EGS4 Monte Carlo determination of the beta dose kernel in water," Med. Phys. 17, 179-186 (1990).

${ }^{217}$ D. J. Simpkin, S. J. Cullom, and T. R. Mackie, “The spatial and energy dependence of bremsstrahlung production about beta point sources in $\mathrm{H}_{2} \mathrm{O}$," Med. Phys. 19, 105-114 (1992).

${ }^{218} \mathrm{P}$. K. Leichner, "A unified approach to photon and beta particle dosimetry," J. Nucl. Med. 35, 1721-1729 (1994).

${ }^{219}$ E. E. Furhang, G. Sgouros, and C. S. Chui, "Radionuclide photon dose kernels for internal emitter dosimetry" Med. Phys. 23, 759-764 (1996).

${ }^{220}$ M. G. Stabin, "MIRDOSE: personal computer software for internal dose assessment in nuclear medicine,' Nucl. Med. Biol. 37, 538-546 (1996).

${ }^{221}$ T. K. Johnson, "MABDOs: a generalized program for internal radionuclide dosimetry," Comput. Methods Programs Biomed. 27, 159-167 (1988).

${ }^{222}$ T. K. Johnson and R. L. Vessella, "On the possibility of 'real-time' Monte Carlo calculations for the estimation of absorbed dose in radioimmunotherapy," Comput. Methods Programs Biomed. 29, 205-210 (1989).

${ }^{223}$ T. K. Johnson and S. B. Colby, "Photon contribution to tumor dose from considerations of ${ }^{131}$ I radiolabeled antibody uptake in liver, spleen, and whole body," Med. Phys. 20, 1667-1674 (1993).

${ }^{224}$ G. Akabani and J. W. Poston, "Absorbed dose calculations to blood and blood vessels for internally deposited radionuclides," J. Nucl. Med. 32, 830-834 (1991).

${ }^{225} \mathrm{G}$. Akabani, "Absorbed dose calculations in Haversian canals for several beta-emitting radionuclides," J. Nucl. Med. 34, 1361-1366 (1993).

${ }^{226}$ M. G. Stabin, "Radiation dose to the upper spine from therapeutic administrations of iodine-131-sodium iodide,' J. Nucl. Med. 34, 695-696 (1993).

${ }^{227}$ R. C. Samaratunga, S. R. Thomas, J. D. Hinnefeld, L. C. von Kuster, D. M. Hyams, J. S. Moulton, M. I. Sperling, and H. R. Maxon, "A Monte Carlo simulation model for radiation dose to metastatic skeletal tumour from rhenium 186(Sn) HEDP," J. Nucl. Med. 36, 336-350 (1995).

${ }^{228} \mathrm{~L}$. S. Johnson and J. C. Yanch, "Calculation of beta dosimetry in radiation synovectomy using Monte Carlo simulation (EGS4),' Med. Phys. 20, 747-754 (1993)

${ }^{229}$ L. S. Johnson, J. C. Yanch, S. Shortkroff, C. L. Barnes, A. I. Spitzer, and C. B. Sledge "Beta-particle dosimetry in radiation synovectomy," Eur. J. Nucl. Med. 22, 977-988 (1995).

${ }^{230}$ H. B. Giap, D. J. Macey, J. E. Bayouth, and A. L. Boyer, "Validation of a dose-point kernel convolution technique for internal dosimetry," Phys. Med. Biol. 40, 365-381 (1995)

${ }^{231}$ G. Akabani, W. G. Hawkins, M. B. Eckblade, and P. K. Leichner, "Patient-specific dosimetry using quantitative SPECT imaging and threedimensional discrete Fourier transform convolution," J. Nucl. Med. 38, 308-314 (1997)

${ }^{232}$ M. Tagesson, M. Ljungberg, and S.-E. Strand, “A Monte Carlo program converting activity distributions to absorbed dose distributions in a radionuclide treatment planning system," Acta Oncol. 35, 367-372 (1996).

${ }^{233}$ H. B. Giap, D. J. Macey, and D. A. Podoloff, "Development of a SPECTbased three-dimensional treatment planning system for radioimmunotherapy," J. Nucl. Med. 36, 1885-1894 (1995).

${ }^{234}$ K. S. Kolbert, G. Sgouros, A. M. Scott, J. E. Bronstein, R. A. Malane, J. Zhang, H. Kalaigian, S. McNamara, L. Schwartz, S. M. Larson, "Implementation and evaluation of patient-specific three-dimensional internal dosimetry," J. Nucl. Med. 38, 301-308 (1997).

${ }^{235}$ E. E. Furhang, C. S. Chui, and G. Sgouros, "A Monte Carlo approach to patient-specific dosimetry," Med. Phys. 23, 1523-1529 (1996).

${ }^{236} \mathrm{~J}$. L. Humm, "A microdosimetric model of astatine-211 labeled antibodies for radioimmunotherapy," Int. J. Radiat. Oncol., Biol., Phys. 13, 1767-1773 (1987).

${ }^{237}$ E. R. Humphreys and J. L. Humm, "A Monte-Carlo approach to the microdosimetry of ${ }^{224} \mathrm{Ra}$ in murine compact and cancellous bone," Health Phys. 54, 607-615 (1988).

${ }^{238}$ J. J. Casciari, M. M. Graham, and J. S. Rasey, "A modeling approach for quantifying tumor hypoxia with [F-18]fluoromisonidazole PET timeactivity data," Med. Phys. 22, 1127-1139 (1995).
${ }^{239}$ P. Millet, J. Delforge, S. Pappata, A. Syrota, and L. Cinotti, “Error analysis on parameter estimates in the ligand-receptor model: application to parameter imaging using PET data,"' Phys. Med. Biol. 41, 2739-2756 (1996).

${ }^{240} \mathrm{C}$. Burger and A. Buck, "Tracer kinetic modeling of receptor data with mathematical metabolite correction," Eur. J. Nucl. Med. 23, 539-545 (1996).

${ }^{241}$ J. A. Thie, G. T. Smith, and K. F. Hubner, "Linear least squares compartmental-model-independent parameter identification in PET," IEEE Trans. Med. Imaging 16, 11-16 (1997).

${ }^{242}$ A. Welch, A. M. Smith, and G. T. Gullberg, "An investigation of the effect of finite system resolution and photon noise on the bias and precision of dynamic cardiac SPECT parameters," Med. Phys. 22, 1829-1836 (1995).

${ }^{243}$ G. L. Zeng, G. T. Gullberg, and R. H. Huesman, "Using linear timeinvariant system theory to estimate kinetic parameters directly from projection measurements," IEEE Trans. Nucl. Sci. 42, 2339-2346 (1995).

${ }^{244}$ P. C. Chiao, W. L. Rogers, N. H. Clinthorne, J. A. Fessler, and A. O. Hero, "Model-based estimation for dynamic cardiac studies using ECT," IEEE Trans. Med. Imaging 13, 217-226 (1994).

${ }^{245}$ R. H. Huesman, B. W. Reutter, G. L. Zeng, and G. T. Gullberg, "Kinetic parameter estimation from SPECT cone-beam projection measurements," Phys. Med. Biol. 43, 973-982 (1998)

${ }^{246}$ K. Ogawa, S. Takahashi, and Y. Satori, "Description of an object in Monte Carlo simulations," IEEE Trans. Nucl. Sci. 44, 1521-1526 (1997).

${ }^{247}$ H. Wang, R. J. Jaszczak, and R. E. Coleman, "Solid geometry-based object model for Monte Carlo simulated emission and transmission tomographic imaging systems," IEEE Trans. Med. Imaging 11, 361-372 (1992).

${ }^{248} \mathrm{H}$. Wang, R. J. Jaszczak, and R. E. Coleman, "A new composite model of objects for Monte Carlo simulation of radiological imaging," Phys. Med. Biol. 38, 1235-1262 (1993).

${ }^{249} \mathrm{~N}$. Amokrane and M. Bourguignon, "A new object model for Monte Carlo simulation of SPECT imaging system," in 1994 Conf. Rec. IEEE Med. Imag. Conf., 1995, pp. 1297-1300.

${ }^{250} \mathrm{Z}$. Li, and J. F. Williamson, "Volume-based geometric modeling for radiation transport calculations," Med. Phys. 19, 667-677 (1992).

${ }^{251} \mathrm{~K}$. Ogawa and S. Maeda, "A Monte Carlo method using Octree structure in photon and electron transport,"' IEEE Trans. Nucl. Sci. 42, 2322-2326 (1995).

${ }^{252}$ G. Williams, M. Zankl, W. Abmayr, R. Veit, and G. Drexler, "The calculation of dose from external photon exposures using reference and realistic human phantoms and Monte Carlo methods," Phys. Med. Biol. 31, 449-452 (1986).

${ }^{253}$ ICRP, "Report of the Task Group on Reference Man," International Commission on Radiological Protection Publication 23 (Pergamon, New York, 1975).

${ }^{254}$ L. G. Bouchet, W. E. Bolch, D. A. Weber, H. L. Atkins, and J. W. Poston, "A revised dosimetric model of the adult head and brain," J. Nucl. Med. 37, 1226-1236 (1996).

${ }^{255}$ W. Q. Sui and F. L. Shen, "Computer model of an inhomogeneous human torso," J. Biomed. Eng. 12, 124-128 (1990).

${ }^{256} \mathrm{~K}$. J. LaCroix, "Evaluation of an attenuation compensation method with respect to defect detection in Tc-99m-sestamibi myocardial SPECT," Ph.D. Dissertation, The University of North Carolina at Chapel Hill, Chapel Hill, NC, 1997

${ }^{257}$ M. Zankl, R. Veit, G. Williams, K. Schneider, H. Fendel, N. Petoussi, and G. Drexler, "The construction of computer tomographic phantoms and their application in radiology and radiation protection," Radiat. Environ. Biophys. 27, 153-164 (1988).

${ }^{258}$ E. J. Hoffman, P. D. Cutler, W. M. Digby, and J. C. Mazziotta, "3-D phantom to simulate cerebral blood flow and metabolic images for PET,", IEEE Trans. Nucl. Sci. 37, 616-620 (1990).

${ }^{259}$ H. J. Kim, B. R. Zeeberg, F. H. Fahey, A. N. Bice, E. J. Hoffman, and R. C. Reba, "Three-dimensional SPECT simulations of a complex threedimensional mathematical brain model and measurements of the threedimensional physical brain phantom," J. Nucl. Med. 32, 1923-1930 (1991).

${ }^{260}$ I. G. Zubal, C. R. Harrell, E. O. Smith, Z. Rattner, G. Gindi, and B. P. Hoffer, "Computerized 3-dimensional segmented human anatomy," Med. Phys. 21, 299-302 (1994).

${ }^{261}$ M. Ivanovic and D. A. Weber, "Monte Carlo simulation code for SPECT 
imaging of uniform and nonuniform media and source distribution,", Nuclear Medicine in Research and Practice, Nuklear Medizine Supp. 28, edited by H. A. E. Schmid and R. Hoffer, 1992, pp. 60-63.

${ }^{262}$ W. R. Nelson, H. Hirayama, and D. W. O. Rogers, "The EGS4 code system,' SLAC-256, Stanford Linear Accelerator Center, Stanford, CA, 1985.

${ }^{263}$ J. A. Halbleib, R. P. Kensek, G. D. Valdez, S. M. Seltzer, and M. J. Berger, "ITS: The Integrated TIGER Series of electron/photon transport codes-version 3.0,' IEEE Trans. Nucl. Sci. 39, 1025-1030 (1992).

${ }^{264}$ J. F. Briesmeister, "MCNP-A general Monte Carlo code for neutron and photon transport," Version 3A, Los Alamos, NM, Los Alamos National Laboratory, LA-12625-M, 1997.

${ }^{265}$ J. C. Yanch and A. B. Dobrzeniecki, "Monte Carlo simulation in SPECT: Complete 3-D modeling of source, collimator and tomographic data acquisition,' IEEE Trans. Nucl. Sci. 40, 198-203 (1993).

${ }^{266}$ M. F. Smith, C. E. Floyd, and R. J. Jaszczak, “'A vectorized Monte Carlo code for modeling photon transport in SPECT,', Med. Phys. 20, 11211127 (1993).

${ }^{267}$ M. F. Smith, "Modelling photon transport in non-uniform media for SPECT with a vectorized Monte Carlo code,' Phys. Med. Biol. 38, 1459-1474 (1993).

${ }^{268}$ A. Passeri, A. R. Formiconi, M. T. De Cristofaro, A. Pupi, and U. Meldolesi, "High-performance computing and networking as tools for accurate emission computed tomography reconstruction,' Eur. J. Nucl. Med. 4, 390-397 (1997).

${ }^{269}$ C. S. Ierotheou, S. P. Johnson, M. Cross, and P. F. Leggett, “'Computer aided parallelisation tools (CAPTools) — conceptual overview and performance on the parallelisation of structured mesh codes," Parallel Comput. 22, 163-195 (1996).

${ }^{270}$ V. C. Bhavsar and J. R. Isaac, "Design and analysis of parallel Monte Carlo algorithms,', SIAM (Soc. Ind. Appl. Math.) J. Sci. Stat. Comput. 81, 73-95 (1987)

${ }^{271}$ J. J. Dongarra, "Performance of various computers using standard linear equations software,' Report CS-89-85, ORNL, Mathematical Sciences Section, 1998.

${ }^{272}$ A. F. Bielajew, D. W. Rogers, “A standard timing benchmark for EGS4 Monte Carlo calculations,'” Med. Phys. 19, 303-304 (1992).

${ }^{273}$ A. J. van der Steen and J. J. Dongarra, "Overview of recent supercomputers,' NCF/Utrecht University Report, The Netherlands, 1998.
${ }^{274}$ A. Geist et al., PVM-A Users' Guide and Tutorial for Networked Parallel Computing (The MIT Press, Boston, 1994).

${ }^{275}$ M. Snir, S. Otto, S. Huss-Lederman, D. Walker, and J. Dongarra, MPI: The Complete Reference (The MIT Press, Boston, 1996).

${ }^{276}$ G. M. Amdahl, "Validity of the single processor approach to achieving large-scale computing capabilities," Proceedings of the American Federation of Information Processing Societies, Washington DC, 1967, Vol. 30, pp. 483-485.

${ }^{277}$ A. De Matteis and S. Pagnutti, "Controlling correlations in parallel Monte Carlo,' Parallel Comput. 21, 73-84 (1995).

${ }^{278}$ R. Sarno, V. C. Bhavsar, and E. M. A. Hussein, "Generation of discrete random variables on vector computers for Monte Carlo simulations,', Int. J. High Speed Comput. 2, 335-350 (1990).

${ }^{279}$ A. Srinavasan, D. M. Ceperley, and M. Mascagni, "Random number generators for parallel applications," in Monte Carlo Methods in Chemical Physics, edited by D. Ferguson, J. I. Siepmann, and D. G. Truhlar, Advances in Chemical Physics Series (Wiley, New York, 1997).

${ }^{280}$ A. De Matteis and S. Pagnutti, "A class of parallel random number generators,' Parallel Comput. 13, 193-198 (1990).

${ }^{281}$ W. R. Martin and F. B. Brown, "Status of vectorized Monte Carlo code for particle transport analysis,' Int. J. Supercomput. Appl. 1, 11-32 (1987).

${ }^{282}$ C. R. Askew et al., "Monte Carlo simulation on transputer arrays," J. Parallel Comput. 6, 247-258 (1988).

${ }^{283} \mathrm{Ch}$. Ma, "Implementation of a Monte Carlo code on a parallel computer system,'” Parallel Comput. 20, 991-1005 (1994).

${ }^{284}$ F. B. Brown and W. R. Martin, "Monte Carlo methods for radiation transport analysis on vector computers,' Progr. Nucl. Energy 14, 269299 (1984).

${ }^{285} \mathrm{~K}$. Miura, "EGS4V: vectorization of the Monte Carlo cascade shower simulation code EGS4," Comput. Phys. Commun. 45, 127-136 (1987).

${ }^{286} \mathrm{~K}$. Miura and R. G. Babb, "Tradeoff in granularity and parallelization for a Monte Carlo shower code (EGS4),', Parallel Comput. 8, 91-100 (1987).

${ }^{287}$ R. G. Babb and L. Storc, "Developing a parallel Monte Carlo transport algorithm using large-grain data flow,', Parallel Comput. 7, 187-198 (1988).

${ }^{288}$ D. R. Kirkby and D. T. Deply, "Parallel operation of Monte Carlo simulations on a diverse network of computers,' Phys. Med. Biol. 42, 12031208 (1997). 